University of Tennessee Health Science Center UTHSC Digital Commons

\title{
Measurement of Autonomic Function in Renal Disease and Diabetes
}

Ann King Cashion

University of Tennessee Health Science Center

Follow this and additional works at: https://dc.uthsc.edu/dissertations

Part of the Endocrine System Diseases Commons, and the Nursing Commons

\section{Recommended Citation}

Cashion, Ann King , "Measurement of Autonomic Function in Renal Disease and Diabetes" (1998). Theses and Dissertations (ETD). Paper 38. http://dx.doi.org/10.21007/etd.cghs.1998.0044.

This Dissertation is brought to you for free and open access by the College of Graduate Health Sciences at UTHSC Digital Commons. It has been accepted for inclusion in Theses and Dissertations (ETD) by an authorized administrator of UTHSC Digital Commons. For more information, please contact jwelch30@uthsc.edu. 


\title{
Measurement of Autonomic Function in Renal Disease and Diabetes
}

\begin{abstract}
Renal disease and diabetes lead to dysautonomia resulting in consequences ranging from gastroparesis to sudden death. New technologies to detect dysautonomia, such as 24 -hr heart rate variability, are being evaluated and compared to traditional evoked tests. These advances have, however, lead to a lack of standardization in testing batteries, procedures, and reporting formats. This series of 3 studies psychometrically assessed measures of autonomic function (AF) and explored relationships among objective and subjective measures in healthy adults and uremic patients. Participants underwent evoked tests that included change in heart rate with deep breathing and Valsalva. In addition, measures of 24-hr HRV (time-domain: SDNN, SDANN, RMSSD; frequency-domain: total power, low and high frequency) and symptomatology were obtained.
\end{abstract}

Study 1 examined the development and psychometric testing of the Autonomic Symptom Checklist (ASC), an instrument designed to assess autonomic symptomatology, with uremic patients $(n=244)$ and healthy adults $(n=34)$. Findings showed the ASC was able to differentiate among healthy and uremic patients with and without diabetes. Test-retest reliability was moderate to high for most categories.

Study 2 established normal, borderline, and abnormal AF values and determined if these values could distinguish healthy $(n=158)$ from uremic adults $(n=363)$. Abnormal values were established at the 2.3 quantile of healthy adults. Uremic patients, especially those with diabetes, had much poorer values than healthy adults. The influence of age and gender on AF measures was attenuated in uremic as compared to healthy adults.

Study 3 examined relationships among and the clinical utility of evoked tests, 24- hr HRV, and the ASC. Data were obtained from pre $(n=130)$ and post $(n=55)$ kidney and kidney-pancreas transplant recipients $(n=130)$, and healthy adults $(n=22)$. The frequency of abnormal values was used to identify the most sensitive measure. Measures of 24 -hr HRV were more sensitive than evoked measures, with frequency measures being most sensitive.

In conclusion, this series of studies established reliability and validity for the ASC, referent values for AF tests, devised a scoring system for AF tests, and found 24-hr HRV measures more sensitive than evoked measures.

\section{Document Type}

Dissertation

\section{Degree Name}

Doctor of Philosophy (PhD)

Program

Nursing

\section{Research Advisor}

Kay F. Engelhardt

\section{Keywords}

heart rate variability, power spectral analysis, sudden cardiac death, symptomatology, transplant, kidney, kidney-pancreas 


\section{Subject Categories}

Endocrine System Diseases | Medicine and Health Sciences | Nursing

\section{Comments}

Two year embargo expired June 2000 


\title{
MEASUREMENT OF AUTONOMIC FUNCTION
}

\section{IN RENAL DISEASE AND DIABETES}

\author{
A Dissertation \\ Presented for \\ The Graduate Studies Council \\ The University of Tennessee, Memphis \\ In Partial Fulfillment \\ Of the Requirements for the Degree \\ Doctor of Philosophy \\ From the University of Tennessee
}

\author{
By \\ Ann King Cashion \\ June 1998
}


Copyright $(\odot$ Ann King Cashion, 1998

All rights reserved 


\section{DEDICATION}

This dissertation is dedicated to my sons

Trotter, Clancy, and Will Cashion

who have given me constant love and support through this endeavor and who believed in me when I doubted myself

and to my husband

Ted Cashion

who has been a source of encouragement toward achieving this goal. 


\section{ACKNOWLEDGEMENTS}

I would like to thank my major professor, Dr. Donna K. Hathaway, for her

guidance, patience and encouragement. Her willingness to nurture my growing scholarly attitude exemplified the mentor role. I would also like to thank my other committee members, Dr. Kay Engelhardt, Dr. Cynthia Russell, and Dr. Jack Buchanan for their comments and assistance over the past three years; Dr. A. Osama Gaber for his clinical and research support; and Dr. William Applegate for his support. A very special thanks goes to my "community of scholars”, especially Rebecca P. Winsett, who encouraged and supported me during my early days as a scholar and have since become valued friends. I would also like to thank my parents, Ann and John King, who have encouraged me throughout and have made it possible for me to attain this goal. In addition, this research study would not be possible without support in part by NIH grant RO1NR03871. 


\section{Measurement of Autonomic Function in Renal Disease and Diabetes}

Renal disease and diabetes lead to dysautonomia resulting in consequences ranging from gastroparesis to sudden death. New technologies to detect dysautonomia, such as 24-hr heart rate variability, are being evaluated and compared to traditional evoked tests. These advances have, however, lead to a lack of standardization in testing batteries, procedures, and reporting formats. This series of 3 studies psychometrically assessed measures of autonomic function (AF) and explored relationships among objective and subjective measures in healthy adults and uremic patients.

Participants underwent evoked tests that included change in heart rate with deep breathing and Valsalva. In addition, measures of 24-hr HRV (time-domain: SDNN, SDANN, RMSSD; frequency-domain: total power, low and high frequency) and symptomatology were obtained.

Study 1 examined the development and psychometric testing of the Autonomic Symptom Checklist (ASC), an instrument designed to assess autonomic symptomatology, with uremic patients $(n=244)$ and healthy adults $(n=34)$. Findings showed the ASC was able to differentiate among healthy and uremic patients with and without diabetes. Testretest reliability was moderate to high for most categories.

Study 2 established normal, borderline, and abnormal AF values and determined if these values could distinguish healthy $(n=158)$ from uremic adults $(n=363)$. Abnormal values were established at the 2.3 quantile of healthy adults. Uremic patients, especially those with diabetes, had much poorer values than healthy adults. The influence of age and gender on AF measures was attenuated in uremic as compared to healthy adults. 
Study 3 examined relationships among and the clinical utility of evoked tests, 24hr HRV, and the ASC. Data were obtained from pre $(\mathrm{n}=130)$ and post $(\mathrm{n}=55)$ kidney and kidney-pancreas transplant recipients $(n=130)$, and healthy adults $(n=22)$. The frequency of abnormal values was used to identify the most sensitive measure. Measures of 24-hr HRV were more sensitive than evoked measures, with frequency measures being most sensitive.

In conclusion, this series of studies established reliability and validity for the ASC, referent values for AF tests, devised a scoring system for AF tests, and found 24-hr HRV measures more sensitive than evoked measures. 


\section{Table of Contents}

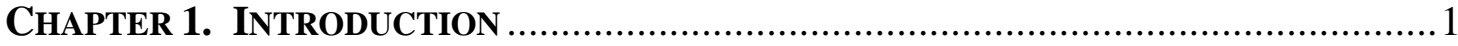

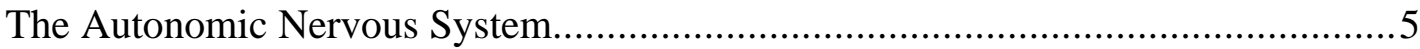

Assessment of Autonomic Function..............................................................

Laboratory Evoked Autonomic Function Measures......................................... 10

24-hr Heart Rate Variability Measures ......................................................... 12

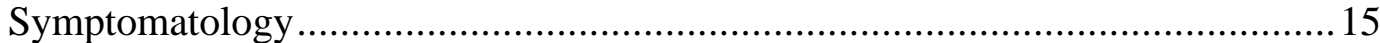

Factors Thought to Influence Autonomic Function........................................ 17

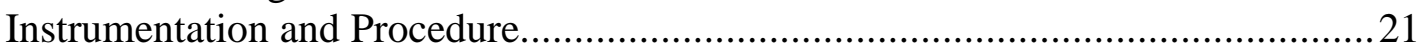

Laboratory Evoked Autonomic Function Measures.................................... 21

24-hr Heart Rate Variability Measures .....................................................2 23

Correlations between Time- and Frequency-Domain Variables ........................28

Autonomic Symptom Checklist ............................................................... 32

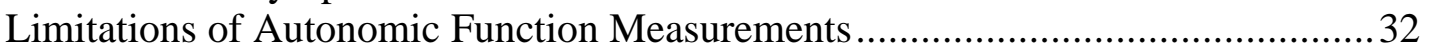

Laboratory Evoked Autonomic Function Measures.........................................33

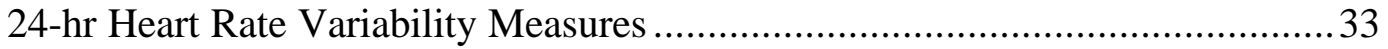

Autonomic Symptom Checklist ..................................................................... 37

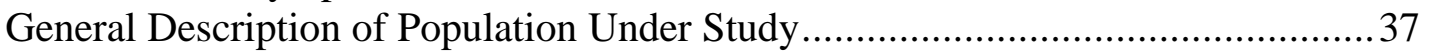

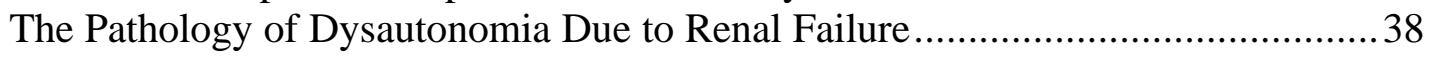

The Pathology of Dysautonomia Due to Diabetes Mellitus ................................ 40

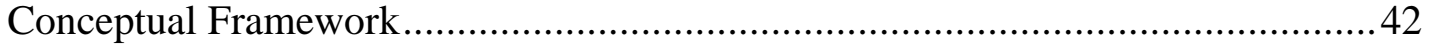

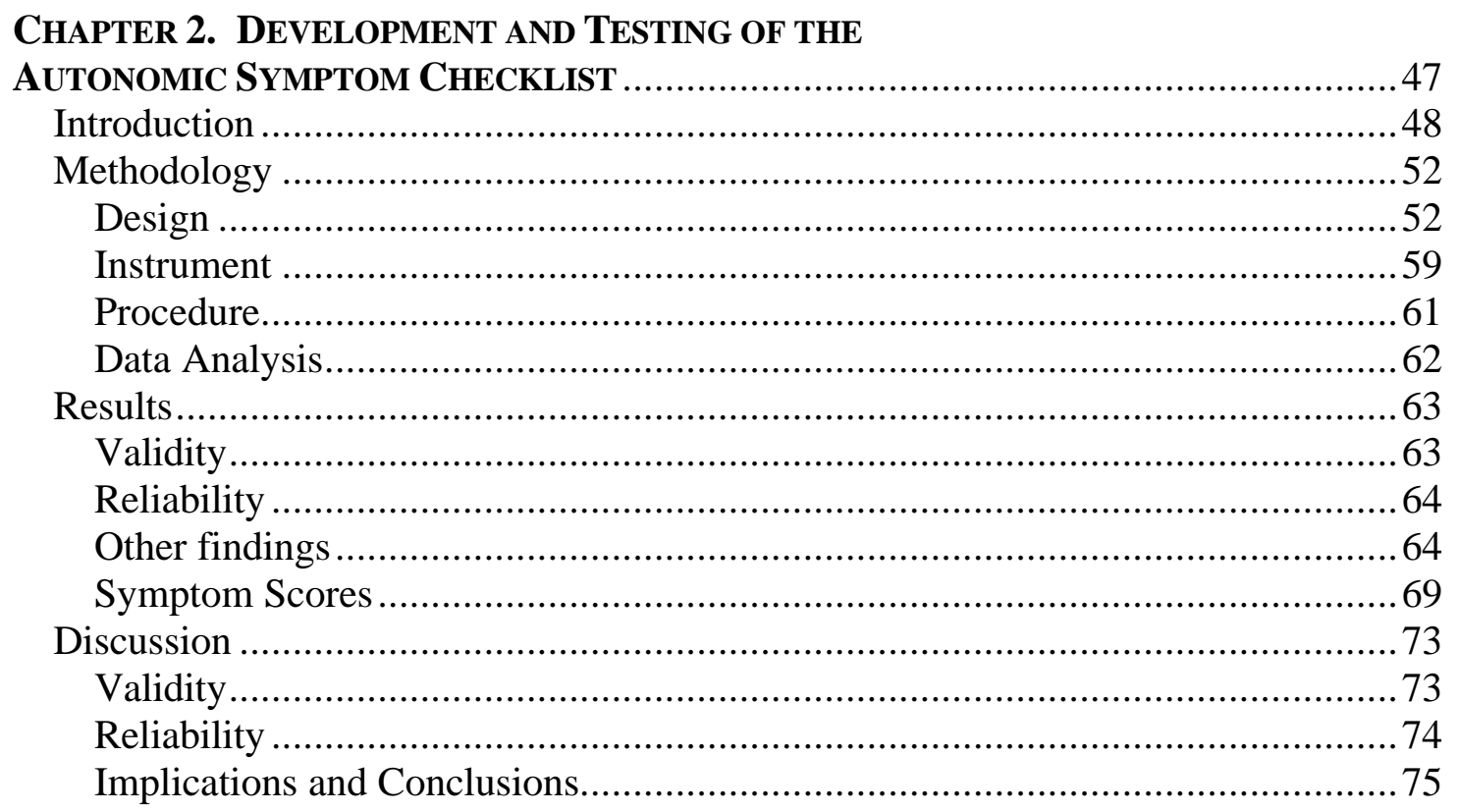




\section{Chapter 3. Establishment Of Referent VAlues for EvoKed Autonomic FunCtion, 24-Hr HEART RATE VARIABILITY, AND AUTONOMIC

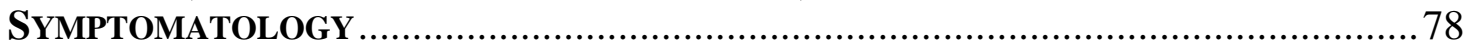

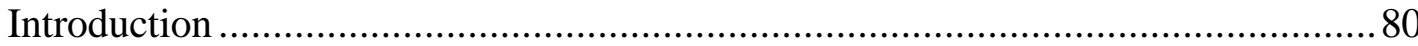

Cardiovascular Autonomic Function Measures ……………………………....... 84

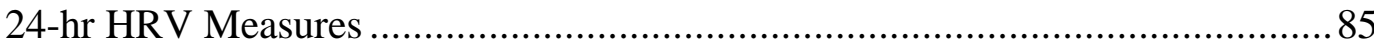

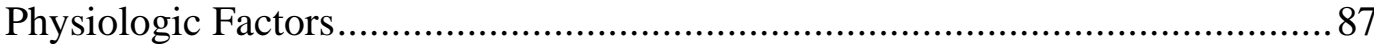

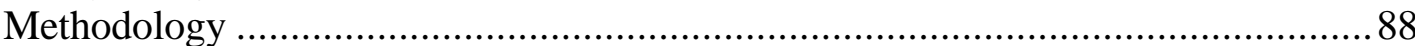

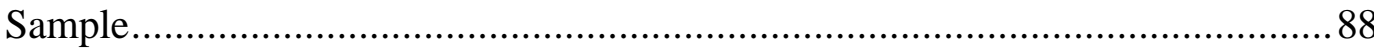

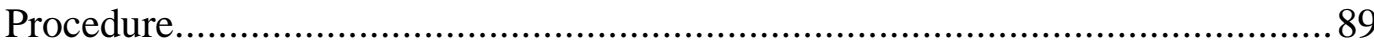

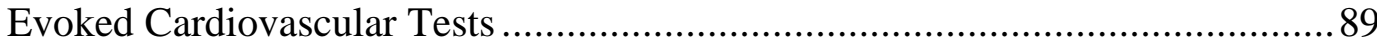

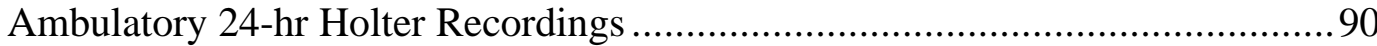

Autonomic Symptom Checklist .................................................................92

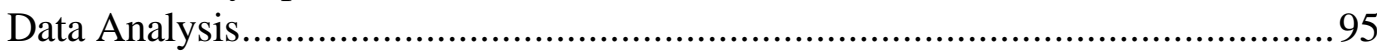

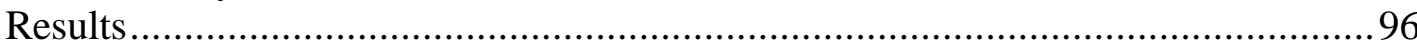

Autonomic Function of Healthy Controls and Two ESRD Samples ....................96

Relationship Between Age and Autonomic Function of Healthy

Controls and Two ESRD Samples.....................................................................99

Relationship Between Gender and Autonomic Function of Healthy

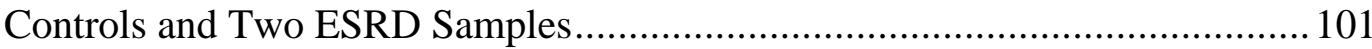

Relationships Among 24-hr HRV Measures of Autonomic Function................. 105

Referent Values for Evoked Measures, 24-hr HRV Measures, and

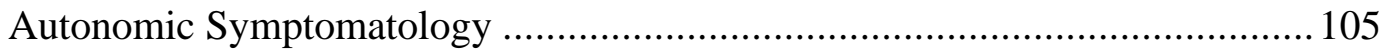

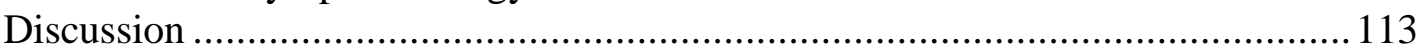

Chapter 4. Correlation ANd Sensitivity Among Three Instruments DESIGNED TO MEASURE AUTONOMIC FUNCTION .............................................. 118

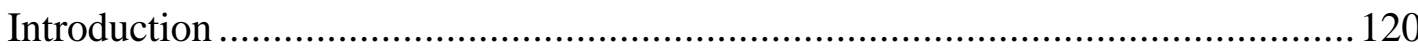

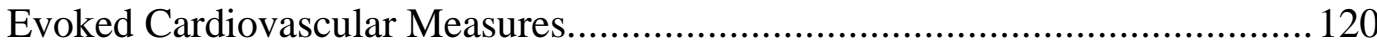

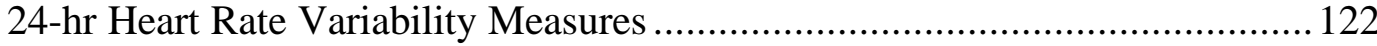

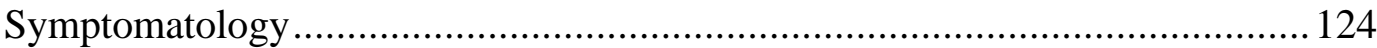

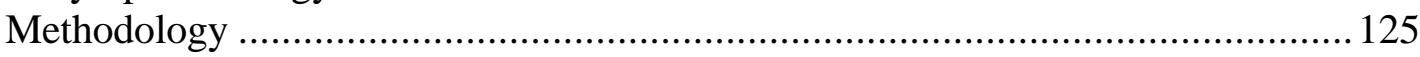

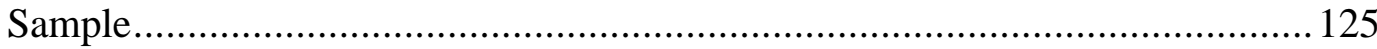

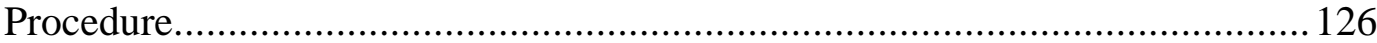

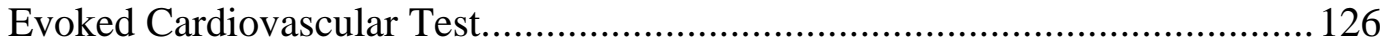

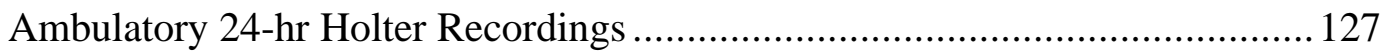

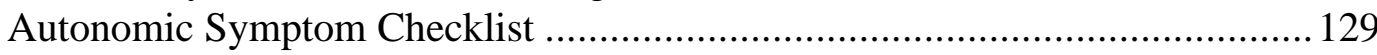

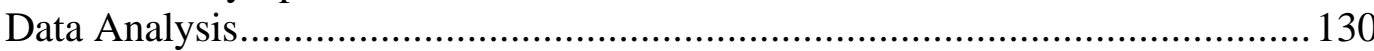

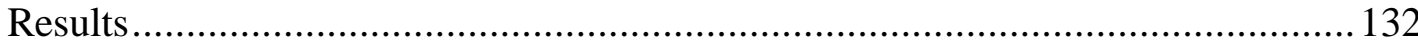

Correlations of Within Test Measures ............................................................... 133

Clinical Utility of Measures of Autonomic Function .......................................... 136

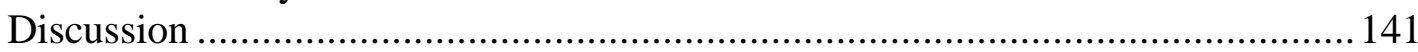


Chapter 5. Summary, Recommendations, ANd Conclusions....................... 147

The Autonomic Symptom Checklist ............................................................. 148

Referent Values for Evoked Tests, 24-hr HRV Monitoring, and the ASC............. 150

Correlation and Sensitivity of Measures of Autonomic Function........................ 151

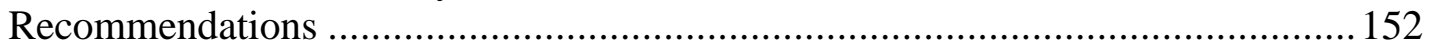

Methodological Recommendations ...................................................... 152

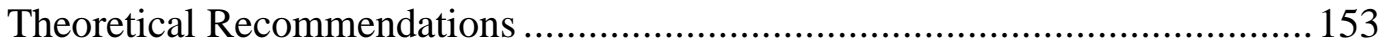

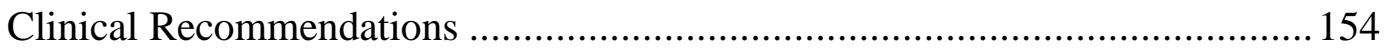

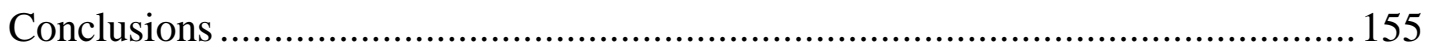

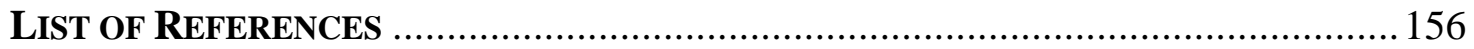

APPENDICES .......................................................................................... 166

Appendix A Testing and Analysis Procedures for Evoked

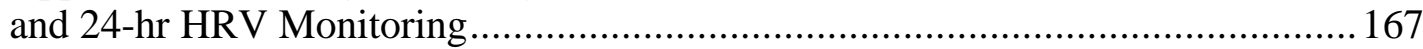

Appendix B Informed Consents ................................................................ 179

Appendix C Autonomic Symptom Checklist.................................................. 187

Appendix D Calculation Procedure for the Autonomic Symptom Checklist ........ 189

VITA 


\section{List of Tables}

Table 1. Definitions of Time- AND FreQuenCy-Domain Measures OF 24-HOUR HEART RATE VARIABILITY AND THEIR RELATIONSHIP TO AUTONOMIC FUNCTION

TABle 2. Characteristics of Healthy CONTROLS, ESRD Patients WITH DiabeTES MELLITUS (DM), ESRD PATIENTS Without DiABETES MELLITUS (NONDM), AND THE TOTAL SAMPLE. 55

TABle 3. Characteristics of MALE Subgroups IN THE Healthy CONTROLS, ESRD PATIENTS WITH DiabeTES MELliTUS (DM), ESRD Patients Without Diabetes Mellitus (NonDM), AND the Total SAMPLE........56

TABle 4. Characteristics OF Healthy CONTROL AND ESRD PATIENT RELIABILITY SUBGROUPS

TABle 5. Characteristics of Hypoglycemia Unaware AND Hypoglycemia AWARE SUBGROUPS 58

Table 6. Symptom and Impact SCores COMPaRing Healthy Controls, PATIENTS WITH ESRD AND PATIENTS WITH BOTH ESRD AND DM ON FOUR SECTIONS OF THE AUTONOMIC SYMPTOM CHECKLIST .65

TABLE 7. SyMPTOM AND IMPACT SCORES FROM THE AUTONOMIC SYMPTOM .66

TABle 8. CORRElations FOR PRE- AND POSTTEST ASC SCORES FOR THREe SYMPTOM CATEGORIES

TABle 9. CORRELATIONS FOR PRE- AND POSTTEST ASC SCORES FOR IMPOTENCE AND HYPOGLYCEMIA CATEGORIES.

TABLE 10. ASC MEAN SyMPTOM AND IMPACT SCORES By GROUP AND GENDER 69 
TABle 11. HigheSt To LoweSt ASC MeAn SyMPTOM AND IMPACt SCORES FOR ESRD PATIENTS BY GROUP AND GENDER 70

TABLE 12. CHARACTERISTICS OF INDEPENDENT SAMPLES AND MATCHED SAMPLES OF PRE AND POSTTRANSPLANT ESRD PATIENTS 71

TABLE 13. ASC CATEGORY MEAN SYMPTOM SCORES AND INDEX SCORES FOR INDEPENDENT AND MATCHED SAMPLES OF PRE AND POSTTRANSPLANT ESRD PATIENTS 72

TABLE 14. VARYING NORMAL, BORDERLINE, AND ABNORMAL VALUES FOR EVOKED MEASURES 82

TABle 15. Characteristic Values For Healthy MidDle-AGed Persons (SElected 24-HR Time- AND FREQUENCY-Domain MEASUReS) 83

TABle 16. Characteristics of Healthy Controls, ESRD PRETRANSPlant PATIENTS, ESRD PATIENTS WITH DiABETES MELliTUS (DM), AND ESRD Patients Without Diabetes Mellitus (NONDM) .98

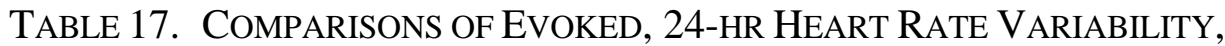
AND ASC MEASURES AMONG HEALTHY CONTROLS (N=158) AND ESRD PRETRANSPLANT PATIENTS (N=363) WITH (ESRD DM=91) AND WithOUT DIABETES (ESRD NONDM=272) 100

TABle 18. Correlational Analysis Between Age And Evoked AND 24-HR HRV MEASURES FOR EACH OF THE THREE STUDY GROUPS 103

TABle 19. COMPaRisons Between Women AND Men OF EVoKed, 24-HR Heart Rate Variability, AND ASC MEasures Among Healthy and ESRD PATIENTS AS A GROUP, WITH (ESRD DM) AND WiTHOUT (ESRD NONDM) DIABETES. 104

Table 20. Correlational Analysis Among Evoked, Time- AND FreQuencyDOMAIN MEASURES OF 24-HR HRV IN HEALTHY CONTROLS ( $\mathrm{N}=158$ ) 106 
Table 21. Correlational Analysis Among EvoKed Measures, TIME- AND FREQUENCY-DOMAIN MEASURES OF 24-HR HRV IN ESRD DM PATIENTS $(\mathrm{N}=91)$ 107

Table 22. Correlational Analysis Among Evoked Measures, TIME- AND FREQUENCY-DOMAIN MEASURES OF 24-HR HRV IN ESRD NONDM PATIENTS (N=272) 108

TABle 23. Number AND Percentage OF ABNORMAL TeSt RESPONSES IN HEALTHY CONTROLS, ESRD DM PATIENTS, AND ESRD NONDM PATIENTS 110

TABLE 24. NORMAL, ABNORMAL, AND BORDERLINE VALUES FOR 2 EVOKED MEASURES, 3 FREQUENCY-DOMAIN MEASURES, 6 TIME-DOMAIN MeAsures, AND THE ASC INDEX BASEd ON THE 2.3 and $5^{\mathrm{TH}}$ PeRCENTILE VALUES OF A GROUP OF HEALTHY INDIVIDUALS (N=158).

Table 25. Correlational Analysis Among Measures in Four Sections OF THE ASC IN PRE AND POSTTRANSPLANT ESRD AND HEALTHY $\operatorname{MEN}(\mathrm{N}=123)$

Table 26. Correlational Analysis Among Measures in Four Sections OF THE ASC IN PRE AND POSTTRANSPLANT ESRD PATIENTS WITH DIABETES MelLitus ( $\mathrm{N}=51)$ 138

TABLE 27. CHARACTERISTICS OF HEALTHY INDIVIDUALS, ESRD PRETRANSPLANT PATIENTS, AND ESRD POSTTRANSPLANT PATIENTS 140

Table 28. Correlational Analysis Among Evoked Score, Time-Domain SCORE, FREQUENCY SCORE, 24-HR HRV SCORE, AND ASC SCORE IN ESRD PRE AND PoSTTRANSPLANT ( $\mathrm{N}=185)$ AND HEALTHY INDIVIDUALS ( $=22)$. 142

TABle 29. SENSitivity of EVoKed, Time, Frequency, 24-HR HRV AND ASC SCORES IN HEALTHY INDIVIDUALS, ESRD PRETRANSPLANT PATIENTS, AND POSTTRANSPLANT PATIENTS. 143 


\section{Chapter 1. Introduction}

Autonomic nervous system dysfunction, dysautonomia, is responsible for incapacitating and life threatening patient outcomes ranging from gastroparesis to sudden cardiac death. Depending on the nature and frequency of the symptoms, dysautonomia can have a devastating effect on quality of life. These conditions are prominent in patients experiencing end-stage renal disease (ESRD), and those who also have diabetes mellitus experience even poorer autonomic function. Cardiovascular autonomic function is of particular concern for patients with ESRD and diabetes as 50\% of this population die within two years of initiating maintenance hemodialysis therapy, many from consequences of cardiovascular complications ${ }^{1}$.

In the last twenty years, a wide-variety of disciplines, including neurology, cardiology, physiology and nursing, have examined autonomic function. This has lead to rapid change and development in the methods to evaluate, manage, and treat dysautonomia. Unfortunately, this growth has also caused a lack in standardization in testing batteries and procedures, in addition to diversity in reporting formats. Thus, sharing of findings and building on the findings of other researchers both within and among disciplines has been hampered.

Currently, numerous studies are examining objective measures of autonomic function in a variety of populations. However, only one study was found that examined and documented subjective symptoms of dysautonomia. In addition, no self-administered instruments with established psychometric properties were available to measure symptoms of dysautonomia. Although symptoms of dysautonomia usually do not occur 
until objective measures are present, it is the symptoms that bring the patient to the health care system. Thus, symptoms of dysautonomia, particularly orthostatic hypotension and impotence, prompt objective testing for dysautonomia and may subsequently lead to treatment. In order to correlate symptoms with objective measures of dysautonomia and to evaluate interventions designed to improve autonomic function, a psychometrically sound instrument designed to measure subjective symptoms of dysautonomia needs to be developed and tested.

Due to the lack of a clear methodology for autonomic measurement and a lack of consistency in analysis and reporting of findings both within and among disciplines, generally accepted referent values for both healthy individuals and patient populations have not been established. Within the literature, individual studies report normal values $^{2}, 3$, but these values are for narrowly defined groups and are frequently based on the autonomic function of healthy adult men. It is generally recommended that each autonomic function lab should establish its own referent values for both healthy and patient populations 4,5 . Thus, referent values for both healthy groups and the ESRD population need to be established.

The rapid and diversified growth in the methods and instruments used to objectively quantify autonomic function has further added to the confusion. With recent technological advances, newer methods of autonomic function testing are being evaluated and findings reported. Some traditionally reported measures are currently being found less sensitive to early changes in autonomic function, while some investigators consider the cutting-edge technology to lack validity. The Physiologic Function Lab at our center has been using two techniques to collect data on physiological measures of autonomic 
function, evoked and 24-hr tests of heart rate variability (HRV). Patient reported symptomatology data have recently been added to the repertoire of tests. Establishment of normal referent values, development of a score system for each group of these measures, and subsequently examining the relationships among all measures and scores with both ESRD patients and a group of healthy controls would permit establishment of validity, and evaluation of sensitivity among the measures. In addition, symptomatology could then be correlated with objective measures of dysautonomia. This would possibly lead to the determination of a threshold where objective measures of autonomic function could predict the development of symptoms. If such a threshold could be found, interventions could be implemented prior to the development of symptoms.

The objective measurement of autonomic function is in a state of change and symptomatology has not been consistently evaluated and correlated with objective measures. Thus, the purpose of this research was to psychometrically assess measures of autonomic function and explore relationships among objective and subjective measurements of autonomic function in normal healthy adults and ESRD patients. Specifically, the aims of this investigation were to:

1. Develop a reliable and valid instrument, the Autonomic Symptom Checklist, to assess symptomatology associated with dysautonomia.

1a) To what extent does the Autonomic Symptom Checklist demonstrate validity by distinguishing among known groups with and without dysautonomia?

1b) To what extent does the Autonomic Symptom Checklist demonstrate reliability in patients with end-stage renal disease and in healthy adult individuals?

1c) To what extent is the Autonomic Symptom Checklist useful as an instrument for 
autonomic symptomatology assessment?

2. Establish evoked autonomic function, 24-hr HRV, and symptomatology referent values for healthy adult individuals and values that are characteristic of patients with end-stage renal disease.

2a) What responses to evoked cardiovascular function testing, 24-hr HRV monitoring, and autonomic symptomatology are characteristic of healthy adult individuals and adult ESRD pretransplant patients?

2b) What are the relationships among age and measures of evoked cardiovascular function, 24-hr HRV monitoring, and autonomic symptomatology for healthy adult individuals and adult ESRD pretransplant patients?

2c) What are the differences among measures of evoked cardiovascular function, 24-hr HRV monitoring, and autonomic symptomatology for healthy adult men and women and adult men and women ESRD pretransplant patients?

2d) What are the relationships among measures of autonomic function in healthy adult individuals and adult ESRD pretransplant patients?

2e) What are normal, borderline, and abnormal values for evoked autonomic tests, 24-hr HRV, and Autonomic Symptom Checklist measures?

3. What are the correlations and sensitivities among evoked tests, 24-hr HRV monitoring, and Autonomic Symptom Checklists?

3a) What are the correlations of within test measures of evoked tests, 24-hr heart rate variability tests, and Autonomic Symptom Checklists?

3b) What are the correlations among evoked scores, 24-hr HRV time- and frequencydomain scores, the 24-hr heart rate variability score, and the Autonomic Symptom 
Checklist score?

3e) What are the clinical utility and sensitivity of evoked scores, 24-hr heart rate variability scores, and Autonomic Symptom Checklist scores?

\section{The Autonomic Nervous System}

The autonomic nervous system (ANS) performs vital regulatory functions using primarily efferent fibers to control multiple organ systems including cardiorespiratory, gastrointestinal, and genitourinary. These systems are largely independent of conscious control, but can be affected by both conscious control and emotional output. In addition, the body uses the ANS to maintain normal homeostasis by regulating body temperature, blood pressure and fluid and electrolyte balance 6,7 .

The multiple functions of the ANS are accomplished through a balance of excitatory and inhibitory impulses which originate from centers in the spinal cord, brain stem, and higher centers and which are transmitted via parasympathetic and sympathetic pathways. The efferent pathways arising from the craniosacral area of the spinal column carry the parasympathetic fibers; those from the thoracolumbar region carry the sympathetic fibers. Both parasympathetic and sympathetic divisions have a central and peripheral component. In addition, in recent years the concept of a central autonomic network, which is located in the brainstem and spinal cord and functions to integrate and regulate the parasympathetic and sympathetic divisions, has become accepted.

The nucleus tractus solitarius located in the medulla oblongata is the area where much of the integration between afferent and efferent fibers occurs. This area obtains input from an individual's internal and external environment and reflexively controls 
body functions; thus, integrating the function of the somatic and autonomic nervous systems ${ }^{7}$. Other areas of the central nervous system, such as the cerebellum and cerebral cortex, have also been shown to play a role in autonomic function?

Within the spinal cord, all descending autonomic fibers project through the intermediolateral or intermediomedial columns. These neurons decrease in number with age at approximately eight percent per decade ${ }^{8}$. Additionally, within these columns somatic sensory neurons release neuropeptides, such as somatostatin and vasoactive intestinal polypeptide, which act as neuromodulators or cotransmitters to modify the neurotransmissions of the ANS. Thus, the somatic sensory system is intimately involved with the ANS ${ }^{8}$.

Both sympathetic and parasympathetic pathways are composed of two types of neurons; preganglionic, which originate in the CNS and are myelinated, and postganglionic, which terminate in the effector organ and are generally unmyelinated 6,7 . The parasympathetic preganglionic fibers are long with short postganglionic fibers; whereas the sympathetic preganglionic fibers are short and synapse with postganglionic fibers in ganglia which are often located near the spinal column and at a distance from the end-organ. Thus, the sympathetic system has much longer postganglionic fibers than the parasympathetic system.

Upon leaving the spinal cord, the peripheral part of the sympathetic nervous system passes through anatomically complex ganglia located on either side of the spinal column. From the numerous sympathetic ganglia, fibers branch to various body organs including the eye, lungs, heart, stomach, pancreas, liver, small and large intestine, kidney and sex organs. In contrast, seventy-five percent of the activity of the peripheral 
parasympathetic nervous system is accounted for by one cranial nerve, the vagus, or Xth cranial nerve. The vagus nerve innervates the lungs, heart, intestine, pancreas, spleen, gallbladder, liver, kidney, bladder and sex organs. Whereas, the other parasympathetic fibers running through cranial nerves III, VII, and IX innervate end-organs primarily within the facial area ${ }^{8}$.

End-organ response to stimulation from sympathetic and parasympathetic fibers is dependent on the neurotransmitters released and the end-organ receptor. Postganglionic parasympathetic synapses release acetylcholine, and are therefore referred to as cholinergic. Postganglionic sympathetic synapses release norepinephrine (noradrenaline) and are referred to as adrenergic. All preganglionic sites, both parasympathetic and sympathetic, predominantly release acetylcholine. The end-organ receptor usually varies primarily by system pathway. The parasympathetic system has at least two types of receptors, muscarinic and nicotinic, and the sympathetic system has primarily alpha and beta receptors.

Physiologically, the body responds differently to sympathetic and parasympathetic stimulation. Sympathetic stimulation causes excitatory effects such as increased sweating, heart rate, and blood pressure while inhibiting peristalsis. Conversely, stimulation of the parasympathetic system causes a decrease in heart rate, and little or no effect on the blood vessels while increasing peristalsis 6,7 . Alterations in parasympathetic and sympathetic function can be quantified by HRV analysis, particularly when using power spectral analysis. Akselrod, Gordon, Ubel, Shannon, Berger, and Cohen ${ }^{9}$ confirmed this in a dog model by alternately augmenting and blocking sympathetic and parasympathetic tone with atropine or vagotomy. The changes 
in autonomic activity were quantified into total (total neural activity), high (primarily parasympathetic activity), and low (primarily sympathetic activity, but some parasympathetic activity) frequency measures of power spectral analysis. This study's results have been reproduced in humans 10 and dogs 11 .

The sympathetic and parasympathetic pathways are also a part of the baroreceptor arc. The baroreceptor arc functions by transmitting signals through the vagal portion of the ANS to the arterial system to enhance vasodilation and decrease blood pressure in response to an increase in arterial pressure. This occurs reflexively when the receptors, primarily located within the aortic arch and the internal carotid arteries, are stretched by an increase in blood pressure ${ }^{6}$. Abnormalities seen within this arc result from modified sensitivity of the arc to changes in arterial pressure. This abnormality can be measured by an altered heart rate response during the release phase of the Valsalva maneuver ${ }^{12}$. In normal individuals, a reflex bradycardia is demonstrated.

Physiologically, when the subject blows against resistance during a simulated Valsalva maneuver, there is an increase in intrathoracic pressure that produces a decrease in venous return with a subsequent decrease in cardiac output. A functioning parasympathetic system responds to the decrease in cardiac output by increasing the heart rate. When the patient quits blowing against the resistance, the intrathoracic pressure decreases allowing an increase in venous return and cardiac output. In addition, peripheral vascular resistance can increase due to augmented sympathetic activity. The resulting increase in systolic arterial pressure is sensed by the baroreceptors in the aortic arch and the carotid arteries. To maintain the body in a homeostatic state, the baroreceptors send out signals decreasing the heart rate. However, if the baroreceptor 
response is blunted, which can occur in the presence of uremia and hyperglycemia, a reflex bradycardia will not occur. Campese and Massry ${ }^{13}$ point out that this is a complex test, and other mechanisms could be involved in the heart rate response to the Valsalva maneuver. Abnormalities could be due to sympathetic dysfunction leading to inappropriate changes in peripheral vascular resistance, derangements in the parasympathetic pathway or an inability of the heart muscle to respond with enough force and volume.

Similarly, a dysfunctional efferent parasympathetic pathway can be assessed by the heart rate response to deep breathing. In patients with uremia, the measured variation in heart rate while breathing slowly and deeply for one minute is reduced ${ }^{13}$. There is no consensus on the role of the efferent sympathetic pathway in uremic patients. Because the ANS has such widespread influence on critical body functions, disruption of the normal balance between the parasympathetic and sympathetic system has the potential to threaten life and well being.

\section{Assessment of Autonomic Function}

Dysautonomia is difficult to measure and quantify because of the dual innervation of the ANS (sympathetic and parasympathetic systems), the numerous reflex arcs involved, and the anatomic dispersion of autonomic nerve fibers ${ }^{14}$. Even with these measurement obstacles, quantification of dysautonomia using objective physiologic measures of autonomic function has been reported in the literature since the $1950 s^{3}, 15-17$. Prior to that time, there were occasional references to clinical signs and symptoms thought to indicate the presence of autonomic neuropathies 18 . In the 1970 s, 
investigators began using a battery of bedside autonomic function tests to aid in interpretation of vague symptoms experienced by both diabetic ${ }^{19}$ and uremic $^{20}$ patients. The battery of tests has moved from the bedside to the laboratory and has lead to improved diagnosis and treatment of autonomic neuropathies at an earlier stage than previously diagnosed ${ }^{3}$. In 1981 , Akselrod et al. ${ }^{9}$ described power spectral analysis of HRV as a quantitative method to evaluate autonomic regulation of the cardiovascular system. Currently, autonomic function is assessed in a variety of ways including laboratory evoked measures, short-term and 24-hr heart rate variability using both timeand frequency-domain measures, and symptomatology. Several physiologic factors, such as gender and age, and confounding variables, such as environmental factors and

smoking, have been identified to possibly have an effect on autonomic function ${ }^{21}$. When interpreting study findings these factors should be taken into consideration.

\section{Laboratory Evoked Autonomic Function Measures}

Evoked measures of autonomic function evaluate heart rate responsiveness to postural and respiratory maneuvers. These tests measure a complex reflex arc reflecting a "beat to beat" balance of sympathetic and parasympathetic activity which can easily be altered by physical and/or emotional stress ${ }^{5}$. Previous studies of cardiac autonomic function have relied primarily on assessment of cardiovascular reflexes using evoked tests, such as the change in heart rate with deep breathing and heart rate variation during Valsalva maneuver 3,22 . Numerous studies $3,20,22-32$ have used these measures to evaluate cardiac autonomic function in normal, uremic and diabetic populations. The high reliability, ease of administration, and non-invasive nature of these tests make them 
the most popular investigative tools ${ }^{3}$. However, there are two major limitations to evoked measures. First, the patient must be willing and able to cooperate with the protocol and second, although a procedure is standardized, stimuli could evoke alternate mechanisms in individuals, thus the resulting measure may not always mean the same thing 33 .

Improvement in autonomic function as measured by Valsalva ratio in ESRD patients with diabetes who have undergone kidney or kidney-pancreas transplantation 34 has been found. Although improvement was also seen in the change in heart rate with deep breathing, it was not as significant as the improvement in Valsalva ratio.

Rothschild, Weinberg, Halter, Porte, and Pfeifer ${ }^{25}$ found that the heart rate change with deep breathing was more sensitive than the Valsalva ratio in detecting parasympathetic ablation and is useful for early detection and follow-up of mild dysautonomia, while the Valsalva ratio may be more useful for sequential long-term evaluation of patients with severe dysautonomia. However, Ziegler, Dannehl, Muhlen, Spuler, Volksw, and Gries 35 found in a study of 130 patients with newly diagnosed type 1 diabetes, that the Valsalva ratio was a more sensitive indicator of poor autonomic function than previously thought. Opfer-Gehrking and Low ${ }^{32}$ suggested that when both measures are decreased it may indicate that there is more cardiac adrenergic failure than previously thought. As these inconsistencies in findings suggest, researchers have been unable to identify one specific measure, such as the change in heart rate with deep breathing or the Valsalva ratio, which consistently reflects changes in cardiac autonomic function. Because there is some inconsistency in the physiologic underpinnings and sensitivity of evoked measures, it is 
important that both measures be included in a battery of tests designed to evaluate autonomic function and monitor its improvement or deterioration 4,21 .

Sensitive measures of autonomic function are needed because impaired vagal activity has been linked to sudden cardiac death in experimental studies ${ }^{36}$, clinical studies $^{37-39}$, and in a small sample of kidney and pancreas-kidney transplant recipients 40,41 . When Campese, Romoff, Levitan, Lane, and Massry 12 investigated the genesis of dysautonomia in four groups (normal controls, predialysis patients, dialysis patients, and patients with chronic illness and normal renal function) using the Valsalva ratio in addition to two tests evaluating sympathetic function, they found that both the predialysis and dialysis patients had dysautonomia with increased dysautonomia in the predialysis group. In addition, recent studies 42,43 show a relationship between reduced vagal activity as measured by a lack of cardiac circadian rhythmicity and cardiovascular events in diabetic populations. Hjalmarson, Gilpin, Nicod, Dittrich, Henning, Engler, Blacky, Smith, Ricou, and Ross ${ }^{44}$ suggest that the lack of an increase in nocturnal parasympathetic function may also provide an explanation for nighttime myocardial infarctions.

\section{4-hr Heart Rate Variability Measures}

Oscillations in heart rate, represented by variations in consecutive R-R intervals, are modulated by sympathetic and parasympathetic outflow. These oscillations can be mathematically estimated by noting how the variations in R-R intervals rhythmically decrease (primarily sympathetic influence) and increase (primarily parasympathetic 
influence) over a given time period. These oscillations in R-R intervals can be examined in both time- and frequency-domains (power spectral analysis).

Twenty-four hour monitoring of heart rate variability and use of power spectral analysis has gained interest as an alternative method to evoked tests for evaluating autonomic function. This method has the potential to assess earlier stages of autonomic cardiovascular dysfunction and examine circadian rhythmicity 42, 45-47. Abnormalities detected by this test are believed to be more sensitive indicators of autonomic dysfunction than evoked tests, particularly in patients with diabetes who have significantly less 24-hour R-R interval variability 48 .

Using power spectral analysis of heart rate variability, studies $49-51$ are dispelling the previously held belief that diabetic autonomic neuropathy primarily affects parasympathetic function. These studies report lower values in measures of sympathetic activity, as well as parasympathetic activity. Similar beliefs were also held regarding the autonomic dysfunction accompanying uremia, and are being dispelled by spectral analysis of heart rate variability 52 .

Short-term recordings of HRV are another technique used to provide physiologic data on autonomic balance. These recordings generally last only minutes (5-20 minutes) and both time- and frequency-domain analyses can be calculated on the electrocardiogram (ECG) recording. Short-term recordings are normally collected while stressing the patient either mentally or physically thus producing a reflex autonomic change. For example, a patient would have an ECG recording made while performing a Valsalva maneuver, changing from a sitting to a standing position, or solving mathematical problems. In response to stresses such as these, a change could be seen in 
autonomic function as measured by variability in R-R intervals. It is thought that shortterm recordings generally reflect modulation of vagal activity 53 .

Very little data has been gathered that compares short-term to long-term recordings. In one such study, Bigger, Fleiss, Rolnitzky, and Steinman ${ }^{54}$ compared mean power spectral analysis values calculated from short-term periods (2-15 minutes) recorded during the day as well as at night to 24-hr HRV recordings. He found that the short-term recording values were similar to the 24-hr values and that some correlations were quite strong $(\geq 0.75)$. In addition, he found these short-term measures to be excellent predictors of sudden death.

Two major advantages to short-term recordings using power spectral analysis are a shorter data collection period and biological data can better comply with the need for data stationarity 55 . Data stationarity is an assumption made during data analysis that the signal, heart period in this example, does not change with time 56 . Short-term recordings are, however, limited in that they only collect data on short-term reflex responses and, thus, may fail to reflect long-term trends or circadian effects on HRV, as seen in diabetic groups 57 .

Long-term (24-hr) recordings examine an individual's background autonomic activity, providing a more complete picture of total autonomic function. In addition, long-term recordings analyze autonomic function at night when vagal function increases and subtle changes in autonomic balance can be detected. When examining psychometric properties of $24-\mathrm{hr}$ HRV measures, Camm 58 found that the positive predictive ability of 24-hr HRV measurement is higher than that of short-term recordings. 


\section{Symptomatology}

Historically, the diagnosis of autonomic neuropathy was based primarily on symptoms including postural hypotension, sweating abnormalities, intermittent diarrhea, gastric fullness, impotence, and hypoglycemic unawareness 18,59 , which generally occur late in a disease process. In the early 1940s, when these symptoms were initially described $^{60}$, limited medical treatment options were available for patients with diabetes and uremia. The life expectancy for patients with these conditions at that time was short, so that many patients did not live long enough to manifest symptoms and, thus, symptoms were thought to be uncommon.

Postural hypotension is a primary symptom of dysautonomia 61 , even though it tends to be a late clinical feature 59 . It possibly indicates a lesion in the baroreceptor arc resulting in a decrease in peripheral vascular resistance ${ }^{12}$ due to a diminished response of the sympathetic vasoconstrictor fibers to the splanchnic bed, muscle and skin 18 . This prevents normal arteriolar vasoconstriction upon postural change from a sitting to a standing position. Symptoms can include dizziness, faintness, visual impairment, or syncope. However, some patients are asymptomatic even when objective measures document a drop of greater than $30 \mathrm{mmHg}$ in systolic blood pressure upon standing 18 . Although an objective test can measure the amount of postural hypotension, the symptoms that accompany this clinical feature are not always present.

Deficiencies in thermoregulation thought to be caused by sympathetic pathway damage result in sudomotor and vasomotor abnormalities 18 . Sudomotor, or sweating, abnormalities generally occur in a pattern of diminished or absent sweating in the lower 
extremities with compensatory increased sweating in the upper trunk and head.

Gustatory sweating, or increased sweating while eating that is unrelated to a response to spicy foods, is a less common symptom ${ }^{59}$. Symptoms of vasomotor thermoregulatory abnormalities present vaguer symptoms of dysfunction than sudomotor abnormalities. In general, vasomotor complaints center around the individual's feet being either too cold or too hot. These symptoms are thought to be caused by a failure to reflexively vasodilate or vasoconstrict in response to body temperature changes 18 .

Hosking, Bennett, and Hampton ${ }^{59}$ reported that even when the entire gastrointestinal system is involved with generalized hypotonic or hypertonic activity, the patient is often asymptomatic. The genesis of gastrointestinal dysfunctions, which can range from esophageal atony to diarrhea, involves damage to the parasympathetic and sympathetic nervous system 18 , in addition to possible autoimmune factors 62 . Diarrhea is the most prevalent symptom within this category 59 . It is fairly characteristic and can last from hours to days, with liquid stooling up to twenty or more times a day. Nocturnal diarrhea and fecal incontinence are common. Constipation is also a frequent complaint of patients with dysautonomia, but it is unknown how this is related to autonomic nervous system function 18 , or if it is related to other factors such as medications the patient is taking.

Symptoms of urogenital system involvement primarily include changes in the ability to control the bladder and impotence 61 . Symptoms of deficient bladder control develop insidiously, progress slowly, and are complex to differentiate from common complications such as a bladder infection. This makes it difficult to evaluate urinary changes as a symptom of dysautonomia. Damage to the parasympathetic nervous system 
causes impotence with loss of penile erection in diabetic men with a prevalence of up to $50 \% 18,59,60$. Data on female sexual function is scant. Other urogenital symptoms that can occur include ejaculation failure and loss of testicular pain to pressure. Both of these symptoms are believed to result from widespread sympathetic denervation to the pelvic $\operatorname{area}^{18}$. Impotence is not always included as a diagnostic feature ${ }^{63}$ because of confounding variables, such as psychogenic factors or the influence of age and mediations ${ }^{59}$, which can affect penile erection.

Hypoglycemia unawareness is the failure of the patient to recognize symptoms of low blood sugar. This involves both parasympathetic and sympathetic dysfunction, however, the symptoms associated with hypoglycemia, including tachycardia and headache, are sympathetic in origin 18 . Thus, hypoglycemia unawareness indicates the progression of autonomic dysfunction from parasympathetic to sympathetic involvement. It is considered a late symptom manifestation of diabetic autonomic neuropathy and can be particularly devastating, resulting in coma and death.

With improved medical treatment options available, patients with uremia with or without diabetes now have a longer life expectancy and reports of symptomatology are becoming more common. However, symptoms of autonomic neuropathy still occur late in the course of the over-riding disease, and once they do occur, the natural course of dysautonomia is one of continued progression with a high mortality rate 61 .

\section{Factors Thought to Influence Autonomic Function}

Variables that have been reported to have an affect on autonomic function are age, gender, exercise training, and medications. Studies have shown that increasing age is 
associated with decreased HRV27, 31, 64, 65. The effect of gender on measures of autonomic function is less consistent than those of age 27,31 . Braune, Auer, SchulteMonting, Schuerbrock, and Lucking 27 studied 137 healthy individuals between 18 and 85 years of age and found that both the valsalva ratio and the change in heart rate with deep breathing were attenuated with increasing age. Values of both measures for subjects over 65 years of age hardly exceeded baseline normals making it difficult to evaluate for dysautonomia in the over 65 age group. However, changes in both measures were not associated with gender. Lishner, Akselrod, Avi, Oz, Divon, and Ravid 50 also found less pronounced differences in frequency measures between diabetic and healthy subjects over age 65 versus subjects under age 65 .

In another study of 557 normal subjects ranging in age from 10 to 83, Low, Deng, Opfer-Gehrking, Dyck, and O'Brien ${ }^{31}$ evaluated the effect of age and gender on heart rate response to deep breathing $(\mathrm{n}=376)$ and Valsalva ratio $(\mathrm{n}=425)$. The results indicated that heart rate response to deep breathing fell with increasing age and was unaffected by gender. In contrast, the Valsalva ratio decreased with age and was lower for women. The investigators suggested these findings indicated that diagnosis of dysautonomia was possible in individuals over 80 years of age.

Cowan, Pike and Burr ${ }^{65}$ found that, in general, 24-hr HRV was significantly lower in healthy women $(n=71)$ compared with healthy men $(n=40)$ in all time- and frequency-domain measures, except for the RMSSD and the PNN50 (see Table 1). However, resting heart rate differences between women and men were found to explain a high proportion of the gender differences of HRV. In addition, all the time- and frequency-domain measures decreased with increasing age of the healthy sample. In 
contrast, no gender effect on 24-hr HRV was seen in a group of 95 surviving sudden cardiac arrest persons (79 men and 16 women) and only the frequency-domain measures decreased with age.

Bigger, Fleiss, Steinman, Rolnitzky, Schneider, and Stein ${ }^{2}$ recruited 274 healthy middle-aged individuals ( $57 \pm 8.2$ years old, $74 \%$ men $)$ and compared the values that were characteristic of the healthy sample to two samples of myocardial infarction patients. One sample of myocardial infarction patients was obtained from the Cardiac Arrhythmia Pilot Study (CAPS) with measures taken one year post-infarction $(n=278,58 \pm 7.4$ years old, $84 \%$ men). The second sample was obtained from the Multicenter Post Infarction Program (MPIP) with measures taken about 2 weeks post-infarction $(n=684,58 \pm 7.5$ years old, $76 \%$ men). The values established by Bigger et al. were later accepted as normal values by the Task Force of the European Society of Cardiology and the North American Society of Pacing and Electrophysiology 53 . In addition to establishing normal values for 24-hr time- and frequency-domain measures, the purpose of Bigger et al.'s study was to clarify the extent to which age and gender affected 24-hr time- and frequency-domain measures. In the healthy sample, findings showed a significant decrease in low and high frequency measures with increasing age, with low power decreasing $22 \%$ per 10 years and high frequency decreasing $10 \%$ per 10 years. In contrast, when a sample of patients with chronic coronary heart disease was examined there was little relationship found between frequency measures and age. Patients with a recent myocardial infarction, however, demonstrated a strong relationship between frequency measures and age. Thus, while normal individuals and a group with recent autonomic dysfunction continued to demonstrate differences in autonomic function by 
age, a group with chronic autonomic dysfunction did not. For healthy persons, low power was significantly higher for men than for women, however, no differences were seen for total power or high power frequency measures. In contrast, both samples of patients with myocardial infarction demonstrated differences in total power and low power, but not high power frequency measures.

The effect of exercise training on cardiac autonomic function has had conflicting results; however, this may be due to HRV measures being determined using brief intervals of time and various analysis methods 66 . Heart rate variability measures of 20 cardiac subjects were studied using 24-hr Holter monitoring and spectral analysis before and after an 11-week cardiac rehabilitation program, which included exercise ${ }^{67}$. This study concluded that HRV is not changed in cardiac subjects who undergo exercise training. Conversely, in two cross-sectional studies, one comparing healthy male runners $(n=72)$ who were age matched with a sedentary healthy male cohort $(n=72)$, the other comparing healthy endurance trained men $(n=8)$ with healthy age matched untrained men $(n=8)$, the results suggested that the physically active group had significantly higher fitness levels which were associated with significantly higher levels of HRV. Thus, these two studies concluded that long-term aerobic exercise augments HRV64, 66.

Levy, Cerqueira, Harp, Abrass, Schwartz, Stratton68 initiated a 6-month exercise training program to evaluate the effect of exercise on parasympathetic function as measured by brief intervals of HRV. Eleven healthy young men (ages 24-32) and 13 older men (ages 60-82) had HRV measured at rest, during supine exercise, and after the training program. The findings showed that training increased resting HRV by $19 \%$ in young men and by $68 \%$ in older men. From this, two conclusions were reached; 
parasympathetic activity (high power) decreases with age and intensive exercise training partially reverses this age-associated change in parasympathetic function. Thus, strenuous exercise has been shown to improve HRV, although little data documents the effects of a mild to moderate exercise program and no data could be found examining how an exercise program might influence HRV for individuals with known autonomic dysfunction secondary to chronic renal disease.

Beta-adrenergic blocking agents have been shown to increase HRV by simultaneously decreasing adrenergic activity and increasing vagal activity 69 . Antiarrhythmic agents, such as quinidine and amiodarone, which depress parasympathetic activity ${ }^{69}$ have been shown to decrease $\mathrm{HRV}^{70}$ in the frequency-domain of $24-\mathrm{hr} \mathrm{HRV}$ analysis, but not in the time-domain, even in groups on low-dose beta blockers in

combination with anti-arrhythmic medications ${ }^{71}$. In addition, preliminary data 72 obtained from kidney transplant recipients show that mycophenolate mofetil, an immunosuppressant, may interact with beta-blockers to enhance patients' HRV. Further study needs to be done to confirm these findings.

\section{Instrumentation and Procedure}

\section{Laboratory Evoked Autonomic Function Measures}

Noninvasive laboratory evoked tests of cardiovascular autonomic function are typically performed in a temperature-controlled laboratory dedicated to performing autonomic function tests. The room is maintained at $25-27$ degrees centigrade and environmental stimuli such as noise and light are minimized. The laboratory at our 
center is equipped with a Power Macintosh 9500 computer system and AcqKnowledge ® III BIOPAC software for data acquisition and analysis of standard electrocardiogram recordings and blood pressure measurements obtained from a Dinamap ${ }^{\mathrm{TM}} \mathrm{XL}$ (CRITIKON). The protocols (see Appendix A) used to perform the evoked tests were modified from Mayo Clinic's protocols 73 , and a brief overview follows.

Following University Institutional Review Board approval and obtainment of informed consent (see Appendix B), patients are brought into the laboratory, demographic information obtained, testing procedures explained, and monitoring equipment applied. After a 10- to 15-minute period of stabilization of heart rate, blood pressure and skin temperature, laboratory evoked autonomic function tests are performed. Cardiovascular autonomic function is determined by two tests that primarily reflect parasympathetic function, the heart rate change with deep breathing (HRDB) and heart rate change with Valsalva maneuver (VR).

The first of these tests measures the change in heart rate with deep respiration (HRDB). This measure is obtained during a one-minute period of deep breathing (6 breaths/minute) which follows a two-minute period of regular breathing. It is calculated by subtracting the lowest heart rate from the highest heart rate during the 1-minute deep breathing period (see Appendix A). The normal HRDB is abolished by parasympathetic blockade with atropine, but unaffected by sympathetic blockade with propranolol ${ }^{74}$. Thus, HRDB loss is thought to primarily reflect parasympathetic dysfunction.

The second test, the VR, is derived by dividing the highest heart rate during a forced expiration of $40 \mathrm{~mm} \mathrm{Hg}$ for 15 seconds by the lowest heart rate immediately after the maneuver (see Appendix A). The VR can be pharmacologically blocked by atropine, 
but not with cardiac sympathetic blockade ${ }^{17}$. Thus, it is thought to primarily reflect parasympathetic function, but may also reflect some adrenergic function.

A lengthy and rigorous series of developmental, standardization, and reliability/validity studies have been conducted on the evoked cardiovascular tests in

numerous centers. Procedures have been refined 3,5 , normal referent values have been determined $3,19,28$, and reliability and validity have been established for change in heart rate variability with deep breathing and Valsalva ratio $5,19,63$. In one study, the reproducibility of the HRDB was measured by calculating the difference between two tests performed less than two months apart on the same subject. Of the 59 diabetic patients with abnormal results, $97 \%$ remained abnormal or borderline at the second testing 63 . Since various methodologies are employed among centers, it has been recommended that each center establish their own referent values ${ }^{4,5}$.

\section{4-hr Heart Rate Variability Measures}

Heart rate variability measurements in both time- and frequency-domains are obtained by analysis of 24-hr ambulatory Holter monitor tapes. Our laboratory employs Marquette Electronics Laser SXP® Ambulatory ECG Analysis and Editing Systems with version 5.8 software program and Series 8500 Holter recording system. Each QRS complex is digitized, identified and labeled. The analyzed data file is then scanned and manually edited to locate and correct any errors in QRS labeling that would adversely affect measurement of heart rate variability. Tapes generally have $\geq 23$ hours of analyzable data. Using these data files, 24-hr heart rate variability with power spectral analysis is calculated. 
The time-domain analysis of heart rate variability can be divided into two general categories (see Table 1). The first category is derived directly from the R-R intervals and includes means and standard deviations of the interval. Measures in this category of the time-domain include the SDNN, SDANN, and SD. The SDNN is the standard deviation of all R-R intervals during the 24-hours and has been found to be associated with sudden cardiac death 37 . The SDANN is the standard deviation of the means of R-R intervals found in successive five minute blocks over 24-hours and is considered the best measure of overall autonomic balance and represents circadian rhythmicity of autonomic function 75 . The SD is the mean of the standard deviation of the R-R intervals of each 5minute block and is sensitive to variability within the 5-minute block. The second category of time-domain variables is based on the differences between adjacent R-R intervals and includes the PNN50 and the RMSSD. The PNN50 is the proportion of adjacent R-R intervals having a difference $>50$ milliseconds (ms). The RMSSD is the square root of the mean of the sum of squares of differences between adjacent R-R intervals. Both the PNN50 and the RMSSD are virtually independent of circadian rhythms and reflect alterations in autonomic function that are primarily vagally mediated $^{57}$.

Because time-domain measurements are calculated using, in general, three different techniques, the resulting values represent three different views of heart rate variability. The SDNN is based on each R-R interval over the 24-hr period and, thus, is an easy way to calculate overall heart rate variability. The SD and SDANN values are both based on 5-minute periods and reflect long-term oscillatory components of heart rate 
variability. The short-term components of heart rate variability are expressed by the PNN50 and the RMSSD 55 .

Recently, it was recommended that four measures be used for time-domain HRV assessment; SDNN, HRV triangular index, SDANN, and RMSSD 53 . The HRV triangular index is the measure reported from geometric analysis of the electrocardiogram. Because both the SDNN and the HRV triangular index are estimates of overall HRV 53 , and the SDNN is more routinely reported in the literature ${ }^{37}$, the series of studies presented in this dissertation will report SDNN in place of HRV triangular index. In addition, the series of studies is part of a longitudinal research protocol funded by the National Institute of Health and is in compliance with the study measures as defined there 76 . 
Table 1. Definitions of Time- and Frequency-Domain Measures of 24-Hour Heart Rate Variability and Their Relationship to Autonomic Function

\section{TIME-DOMAIN}

Variability of R-R intervals over 24-hours

- SDNN is the standard deviation of all R-R intervals for the entire recording. It is mathematically equal to total power of spectral analysis, thus, reflects all cyclic components responsible for variability in the recording period 53 .

- SDANN is the standard deviation of the averages of R-R intervals calculated over short periods, usually 5-min. blocks, for the entire recording. This is an estimate of the changes in heart rate due to long cycles (> $5 \mathrm{~min}.)^{53}$

- SD (also called the SDNN index) is the mean of the standard deviation of the R-R intervals of each 5-min block for the entire recording. This measures variability due to short cycles $(<5 \mathrm{~min} \text {. })^{53}$

Differences in adjacent R-R intervals (thought to be vagally mediated, they estimate high-frequency variation and, thus, are highly correlated 53 )

- $\quad$ PNN50 is the proportion of adjacent R-R intervals having a difference $>50$ milliseconds

- RMSSD is the square root of the mean of the sum of squares of differences between adjacent R-R intervals

\section{FREQUENCY-DOMAIN}

- total (0.01-1.00 Hz) reflects all cyclic components responsible for variability 53

- low (0.04-0.15 Hz) represents primarily sympathetic activity with some parasympathetic activity modulated by baroreflex activity 77

- high (0.15-0.40 Hertz) represents primarily parasympathetic activity 53 
Calculation of time-domain values is a simple process, unfortunately, when the heart period is reduced to simple means and standard deviations information is lost. For example, there is no direct relationship to a time axis or anyway to differentiate between parasympathetic or sympathetic function using frequency content. To overcome these shortcomings, more sophisticated techniques for analysis have been developed. One of the newest technologies uses signal-processing methods to determine a frequency waveform depicting heart rate variability. It is thought that this method allows further delineation of the beat to beat variabilities into their respective autonomic genesis, thus allowing more in-depth physiological inquiry 78,79 .

The two basic signal-processing models are fast Fourier transform (FFT) and autoregressive (AR) analysis. Fast Fourier transform describes a signal (heart rate pattern) as the sum of sinusoids at fixed and equally spaced frequencies 45,79 . This analysis is dependent on two primary assumptions, stationarity of data and smoothing. There is well-developed nonparametric statistical theory used in FFT computations, even though some 80 consider it statistically unstable. In contrast, AR analysis is based on a linear prediction model derived from the signal (heart rate pattern) itself and does not depend on the same assumptions used in $\mathrm{FFT}^{79}$. Autoregression is a conceptually simple algorithm, but its computational complexity is greater than FFT. Cowan, Burr, Narayanan, Buzaitis, Strasser, and Busch ${ }^{81}$ compared the spectral density of HRV using both AR and FFT techniques and found that either would be methodologically acceptable for clinical studies. The series of studies presented here used FFT to examine periodic oscillations in R-R intervals over a $24-\mathrm{hr}$ period. 
Increases and decreases in R-R interval widths reflecting periodic fluctuations in sympathetic and parasympathetic function are transformed by FFT into a frequency waveform. This technique is frequently referred to as power spectral analysis. The spectrum of frequencies $(\mathrm{Hz})$ present over the 24-hrs is plotted and values provided which indicate the relative amount of total $(0.01-1.00 \mathrm{~Hz})$, low $(0.04-0.15 \mathrm{~Hz})$ and high $(0.15-0.40 \mathrm{~Hz})$ frequency power (see Table 1). Low frequency waveforms estimate sympathetic activity with some parasympathetic activity while high frequency waveforms, also known as the respiratory frequency, represent parasympathetic activity. The ratio of low frequency to high frequency power provides a useful quantification of the balance between the sympathetic and parasympathetic activity. In addition to the 24hour average, power spectral analysis provides an hourly sum of each 5-minute interval throughout the day. These hourly profiles permit visual depiction of the autonomic modulation of circadian rhythms. Normally, low frequency activity (sympathetic activity) predominates during waking hours and high frequency activity (parasympathetic activity) predominates during sleep; thus, a higher ratio, as seen during the awake hours, reflects lower vagal activity.

\section{Correlations between Time- and Frequency-Domain Variables}

Several 24-hr HRV time-and frequency-domain measures are strongly correlated with each other 46,82 . It is thought that these strong correlations exist because of both mathematical and physiological relationships among time- and frequency-domain measures ${ }^{53}$. Kleiger, Bigger, Bosner, Chung, Cook, Rolnitzky, Steinman, and Fleiss 46 obtained 24-hr HRV measures from 14 healthy individuals (20-55 years of age) and found that certain time- and frequency-domain variables are highly correlated concluding 
that they may serve as surrogates for each other. High frequency power, RMSSD, and PNN50, all considered measures of vagal function, were strongly correlated $(r>0.9)$ with each other and with SD. Low frequency power was also strongly correlated with the measures of vagal function $(r>0.8)$ indicating that some parasympathetic activity is reflected in low frequency power.

Bigger, Fleiss, Steinman, Rolnitzky, Kleiger, and Rottman 82 examined the correlations between time- and frequency-domain measures in 24-hr HRV recordings obtained 2 weeks after myocardial infarction $(n=715)$. They found that there were natural groupings for several of the 24-hr measures. From Bigger et al.'s data, Keehn deduced that both SDNN and SDANN are essentially equivalent to total power 83 (see Table 1 for measure definitions). It was suggested 82 that these measures could act as surrogates for each other. Similar patterns of correlation among measures of parasympathetic function (RMSSD, PNN50, and HF) were reported in an earlier study 84 and in a later, larger study $^{2}$. In addition, the SDNN and the square root of total power are almost perfectly correlated, and the time-domain measure, SDNN, was found to be strongly and significantly associated with mortality in this group $2,37$.

Alterations in parasympathetic and sympathetic function can be quantified by HRV analysis, particularly when using power spectral analysis. Akselrod et al. ${ }^{9}$ confirmed this in a dog model by alternately augmenting and blocking sympathetic and parasympathetic tone with atropine or vagotomy. The changes in autonomic activity were quantified in the total (total neural activity), high (primarily parasympathetic activity), and low (primarily sympathetic activity, but some parasympathetic activity) 
frequency measures of power spectral analysis. This study's results have been reproduced in humans 10 and dogs 11 .

Quality assurance data provided by the Marquette Electronics illustrates mathematically correct results following submission of known electronically generated cardiac signals 83 . This technology has been successfully employed in clinical studies evaluating alterations in autonomic regulatory mechanisms in patients with heart disease $^{38}$, cardiac transplantation 85 , diabetic autonomic neuropathy $42,48,86$ uremia 52,87 and kidney transplantation 88 . Twenty-four hour measures have been highly stable in both normal subjects 46 , post myocardial infarction patients 55 , and patients with ventricular arrhythmias 89 . Thus, it is thought that $24-\mathrm{hr}$ measures may be ideal for assessing intervention therapies ${ }^{53}$, such as transplantation, exercise, and deep breathing relaxation techniques.

Before a new technology is approved by the FDA for clinical use, findings from a sufficient number of research studies must support the new technology's efficacy and safety. The psychometric properties, including validity issues of sensitivity and specificity, need to be delineated. Sensitivity is the probability of testing positive if the disease is truly present, and specificity is the probability of testing negative if the disease is truly absent 90 . Another closely related test is predictive value, which is whether the individual actually has the disease, given the results of the test. Twenty-four hour HRV with power spectral analysis is a potential screening test because it is relatively inexpensive, easy to administer and imposes minimal discomfort on the patients. The validity of HRV is determined by its ability to do what it is supposed to do; that is, to 
distinguish between individuals with diminished cardiac autonomic function (who should test positive) and individuals with normal cardiac autonomic function (who should test negative). For example, in the ESRD population, as 24-hr HRV sensitivity increases more patients who have dysautonomia are correctly identified as such. Using the same example, as specificity increases fewer patients with normal autonomic function are falsely identified with dysautonomia (false positive). The goal with instrument design or selection is an instrument with high sensitivity and high specificity; however, this is usually not possible 90 because of tradeoffs between sensitivity and specificity (type 1 and type 2 errors, respectively).

In a report on studies which used 24-hr HRV as a marker of prognosis after myocardial infarction 47 , the sensitivity of HRV measures ranged from $33.9 \%$ to $100 \%$ and specificity ranged from $44.6 \%$ to $92.6 \%$, while the positive predictive value of $\mathrm{HRV}$ was about $40 \%$ when used in combination with other postinfarction risk factors. Malik 91 found when the SDNN, the standard deviation of duration of RR intervals, was $<50 \mathrm{~ms}$, the sensitivity was $46 \%$ and the positive predictive value was $34 \%$ for the prediction of 24 month cardiac mortality. These findings are congruent with Kleiger's ${ }^{37}$ results. Thus, HRV measurements with power spectral analysis can be used as a prognostic indicator for risk stratification of post myocardial infarction ${ }^{92}$, and potentially with other populations such as patients with diabetes and $\mathrm{ESRD}^{93}$, of significant autonomic dysautonomia similar to that of myocardial infarction patients 40,87 . Diminished measures of autonomic function are also found in uremic patients. Only preliminary data 
on sensitivity and specificity for 24-hr HRV measures were found for the ESRD population 93 .

Because 24-hr HRV monitoring with power spectral analysis is being evaluated for clinical use, high sensitivity is required, even if the positive predictive accuracy is not high. In contrast to traditional methods of detecting autonomic dysfunction, 24-hr HRV monitoring has the potential to be an early, accurate, dynamic, screening instrument for the renal transplant population.

\section{Autonomic Symptom Checklist}

Symptoms of autonomic nerve damage are often non-specific and vary depending on the individual. Symptoms generally occur after objective test measures, such as the change in heart rate with deep breathing or the frequency domain measures of 24-hr HRV, indicate a decline in autonomic function61. Recognizing the difficulty of objectively evaluating a person's subjective symptoms, an instrument entitled the Autonomic Symptom Checklist was developed and tested as part of this dissertation. The development of the Autonomic Symptom Checklist is described in Chapter 2.

\section{Limitations of Autonomic Function Measurements}

Autonomic function testing is used in both research and clinical settings. For the purposes of this dissertation, limitations and problems associated with examining autonomic function will be research-oriented, although, many of the limitations apply in the clinical area. The essential attributes for autonomic measurement are that the tests use noninvasive techniques, measure with precision and accuracy, and deliver reproducible results. 


\section{Laboratory Evoked Autonomic Function Measures}

For laboratory evoked autonomic function measurements to be accurate, the tests must be completed using a standardized methodology that requires patient participation. For example, to obtain an accurate VR the patient must be capable of and willing to blow into a mouthpiece against resistance for 15 seconds. Thus, limitations of evoked bedside measures are the patient's ability and willingness to cooperate.

Another significant limitation is that evoked measures do not directly assess the autonomic nervous system. Researchers rely on indirect methods based on animal ${ }^{9}$ and human models 10 to assess autonomic function. Based on the results of animal and human models, the Valsalva maneuver and change in heart rate with deep breathing are theorized to evaluate parasympathetic function. The complexity of the underlying physiology is a limitation of indirect measurements ${ }^{5}$.

\section{4-hr Heart Rate Variability Measures}

Limitations of 24-hr HRV with power spectral analysis result from problems in obtaining and processing of the ECG signal, analyzing the ECG signal process, and interpreting what the components (LF and HF) mean. Twenty-four hour HRV measures use signal processing to evaluate cardiac autonomic function. Biomedical signals convey information about physiologic processes, such as the heart rate, and can be used to evaluate the biologic system. However, acquisition of a signal is not sufficient. To obtain the information needed a processor transforms the signal using various mathematical computations and statistical procedures. Depending on the method of analysis, certain assumptions about the data are made and these assumptions can lead to 
limitations of the measurements. These limitations of the process need to be understood and minimized to the extent possible.

One limitation is digital processing that occurs during data acquisition 53 . The data is digitally processed in an effort to clean the signal of noise 94 . Relevant information, such as frequency, is not readily visible in a noisy signal. Noise usually occurs due to background electrical activity, such as muscle movement, which masks the waveform produced by the autonomic nervous system. Digital processing identifies the background electrical activity and removes it from the signal. Methods to remove noise include traditional linear filtering, which enhances the signal, and optimal filtering, which smoothes the signal with an averaging technique when the signal and the noise overlap and enhancing cannot be accomplished 80 . The continuous signal is then converted into a discrete-time series and quantified by assigning amplitude values to each sample. Both processes change the characteristics of the signal and could affects the analysis 95 . Another assumption is that no data exists outside the recording window. Because data may exist outside the window, this assumption leads to spectral leakage in the analysis. Techniques used to solve this problem can lead to a reduction in frequency resolution resulting in reduced accuracy of frequency measurement.

Stationarity of data relates to signal processing and is the assumption that the signal is present at all times and does not change with time 56 . Assuming stationarity of data simplifies the analysis process and is used when analyzing HRV in both time- and frequency-domains. Non-stationary processing methods are complex or involve cutting data into short durations to assure stationary. Stationarity of data, however, is seldom true when evaluating biological variables, and is not true when analyzing heart rate 
patterns that are generally in a constant state of change. Because infinite data sequences are assumed and the record is finite, estimation errors are introduced 95.

Heart rate is not stationary data because it is constantly affected by balance between the parasympathetic and sympathetic branches of the autonomic nervous system, hormonal, and other physiologic feedback mechanisms. However, the sympathovagal balance is of primary concern in patients with ESRD and diabetes mellitus. Evaluating the HRV over a 24-hr period can provide an in-depth evaluation of the daily modulations occurring in the autonomic nervous system. The researcher can evaluate the effect of such variables as changes in position, changes in respiratory rate, exercise, circadian rhythms, sleeping and eating on fluctuations in HRV. Some researchers use short-term recording of $\mathrm{HRV}$ in order to increase control of data acquisition. When the subject is placed in a controlled environment for the duration of the data acquisition, stationarity of data increases. However, the researcher using 24-hr HRV assessments wants to evaluate the patient's autonomic function during the patients normal lifestyle and activities.

Recommendations 53 have been made that would minimize the effects of nonstationarity of data and other limitations on research investigations. One requirement is a good technical ECG recording for analysis. Error-free and artifact-free recordings are difficult to obtain from ambulatory 24-hr Holter recordings because patients are engaging in everyday activities and movement of skeletal muscles causes artifact on the recordings. Prior to processing, the data needs to be manually-edited to correct mislabeled beats 53 . This results in good quality R-R intervals for analysis. However, over-cleaning the data can reduce frequency resolution and manual editing of long-term ECG recordings is time consuming (1 to 10 hours) and clinically impractical. 
Thus, it is important to follow strict procedures when applying the chest electrodes to obtain higher quality data 83 . Surface contact is critical because data can be affected by muscle movement, electrode motion, and other sources which add noise to the signal. Encouraging the subject to keep an accurate diary of sleep, exercise, eating and other activities during the $24-\mathrm{hr}$ monitoring period will provide additional data when interpreting the HRV measurements. Because medications can affect heart rate fluctuations, obtaining a record of the subject's medications can also be useful when interpreting data. Repeat Holter monitoring is recommended when the data signal is of poor quality, or the subject has an abnormal 24-hours during data acquisition, for example, if the patient was restless due to a thunderstorm.

According to some 96,97 , researchers have made too big a leap when interpreting what different HRV spectral components and time-domain measures mean. It has been established $^{9-11}$ that HF and LF component of HRV are associated with parasympathetic and sympathetic influences, respectively. However, Malik and Camm 96 warn that the HRV components do not reflect autonomic tone, as is frequently assumed. Instead, HF and LF are markers of autonomic influences on the modulations of heart rate. This means that at either maximal stimulation or blockage of parasympathetic or sympathetic activity there is no variability and no spectral components. Thus, when a patient has a reduction in HRV it should be interpreted as a decrease in the modulation of the heart, not necessarily as a decrease in the tone of the autonomic system.

In summary, to diminish limitations associated with 24-hr HRV with power spectral analysis it is important to use recommended techniques 53 when obtaining, processing, and analyzing ECG recordings. In addition, the interpretation of results can 
not be assumed a reflection of autonomic tone, but instead the components represent a modulation in autonomic activity.

\section{Autonomic Symptom Checklist}

A primary limitation of the Autonomic Symptom Checklist is that it is a selfadministered instrument; thus, subject to patient interpretation. In addition, the reliability studies were done using a relatively small sample with very few African-Americans. To date, however, there are no reported studies that have demonstrated significant differences in autonomic function based on race.

\section{General Description of Population Under Study}

Over 11,000 Americans undergo renal transplantation each year with another 800 individuals undergoing kidney-pancreas transplantation. In 1994, diabetes mellitus was the leading cause of renal failure worldwide ${ }^{1}$. Prior to transplant, uremia, especially in combination with diabetes, can cause major alterations in biological function exemplified by severe derangements in autonomic function. The autonomic nervous system exerts widespread control over multiple critical body functions; therefore, the severe autonomic neuropathies that accompany uremia and diabetes can lead to life-threatening cardiac states $38,40,69,75,93,98,99$. Transplantation brings about an immediate correction of uremia and diabetes, which has been found to improve autonomic function 100 and alleviate many of the accompanying symptoms, such as gastropathy and neuropathy 99 .

This series of studies being reported employed a convenience sample of patients with ESRD who were undergoing evaluation for kidney or kidney-pancreas transplantation. In addition, a group of healthy adults was recruited for comparison 
purposes and for establishment of normal referent values. Each patient and healthy adult signed an informed consent (see Appendix B) which gave permission to record autonomic function data and to gather demographic information. The study was approved by the Institutional Review Board of the University of Tennessee, Memphis.

Patients were generally stratified into three groups: those with ESRD who did not have diabetes mellitus (ESRD NonDM), those with ESRD who had type 1 diabetes mellitus (ESRD DM), and healthy individuals. The original diagnoses represented in the non-diabetic ESRD group were hypertension, chronic glomerulonephritis, systemic lupus erythematosus, polycystic kidney disease, ESRD of unknown etiology, and miscellaneous.

\section{The Pathology of Dysautonomia Due to Renal Failure}

The prevalence of dysautonomia in both uremic and dialysis patients was found to be as much as $50 \%$ when evaluated by evoked autonomic function tests 12 . Manifestations of dysautonomia in patients with uremia are primarily thought to be related to increased parathyroid hormone levels ${ }^{101}$, abnormalities within the baroreceptor arc, which includes the efferent parasympathetic pathways, and reduced end-organ responsiveness to vasoconstrictor agonists such as epinephrine ${ }^{12}$. These disturbances are associated with the uremia itself, or consequences of the uremia, and not associated with either anemia or the state of having a chronic illness 12 .

Fraser and Arieff ${ }^{101}$ suggest that there may also be abnormalities in neurotransmission in uremic patients. One potential cause of this is thought to be a change in uremic patients' parathyroid hormone levels. Parathyroid hormone increases 
both intracellular and extracellular levels of calcium and can also affect sodium levels. Both calcium and sodium are intimately involved in neurotransmission along the axon and at the neurosynaptic junction. Changes in the calcium levels can affect enzymes, which are used to provide energy for neurotransmission. In addition, calcium pumps used to mediate neurotransmissions at the nerve terminals can be affected, resulting in abnormalities in processing information 102 . However, researchers have not been able to demonstrate consistently that parathyroid hormone is an uremic neurotoxin 101 , as some have suggested.

In addition, some ${ }^{101}$ have suggested that uremic neuropathy is related to anatomic evidence of nerve damage, such as loss of myelin. Myelin is a fat-like substance that forms a sheath around primarily large-diameter nerve fibers, such as skeletal muscle efferents, and some small-diameter fibers, such as preganglionic sympathetic efferents. Myelin surrounds the nerve fiber and increases conduction rates, thus, loss of myelin would affect electrical conduction of neurotransmission. It is hypothesized that this could affect the baroreceptor arc.

Reduced end-organ response to vasoconstrictor agonists, such as norepinephrine, is thought to play a major role in the manifestations of dysautonomia, particularly in the predialysis patient 12 . It is hypothesized that postural hypotension in the uremic patient is due to down regulation of alpha-adrenergic receptors in the peripheral blood vessels to norepinephrine. The resulting inability to augment peripheral vascular resistance prevents the uremic patient from maintaining a constant blood pressure upon standing. Again, it is unknown what specifically causes this, but these results are consistent with the hypothesis that cumulative effects of multiple uremic toxins decrease 
neurotransmission at the synaptic gap. In addition, excess blood levels of parathyroid hormone could play a factor in end-organ response by stimulating an increase in tissue production of prostacycline ${ }^{12}$.

\section{The Pathology of Dysautonomia Due to Diabetes Mellitus}

Diabetic autonomic neuropathy became recognized as an entity following a comprehensive review by Rundles in $1945^{60}$. Although the pathogenesis, like uremic autonomic neuropathy, has not been established, it is a common complication with a prevalence rate of up to $56 \%$ in patients with long-standing type 1 diabetes $3,22,103$. Even persons with newly diagnosed type 1 diabetes have a prevalence rate of up to $9 \% 35$. Most likely diabetic autonomic neuropathy results from a prolonged exposure to metabolic derangements similar to that postulated for somatic neuropathies 18 .

Metabolic derangements are thought to lead to chronic, widespread neural lesions in the autonomic nervous system with loss of both myelinated and unmyelinated axons 14,104 . Potential factors responsible for axon loss include an accumulation of glucose in the central and peripheral autonomic nerves with potential slowing of conduction, a reduction of myoinositol which is involved with lipid synthesis and reflects a lack of insulin, and changes in myelin composition and synthesis $18,59,105$. Recently autoimmune reactions in both type 1 and type 2 diabetes mellitus have been postulated to cause nerve destruction 106,107 . The hypothesis that diabetic neuropathy results from nerve ischemia brought about by microvascular abnormalities 14,18 is controversial, but is receiving a great deal of investigative attention. Although studies investigating the structural origin of ischemia are inconclusive, the functional evidence of nerve ischemia 
and hypoxia is emerging and may involve the "endothelium-dependent" nitric oxidemediated component ${ }^{14}$.

The Diabetic Control and Complications Trial is a landmark study that investigated the importance of insulin-deficiency, hyperglycemia, or both in the pathogenesis of diabetic neuropathy 108 . The results of this study provided support for the theory that an increase in blood glucose is associated with autonomic neuropathy. Tighter control of hyperglycemia in persons with type 1 diabetes reduced the prevalence of clinical diabetic neuropathy by $60 \%$.

Since both uremic and diabetic autonomic neuropathy have a fragmentary pathogenesis and several commonalties, it is difficult to distinguish between manifestations that are uremic versus diabetic in origin. As seen with dysautonomia due to uremia, the clinical presentation of diabetic autonomic neuropathy is frequently nonspecific and varying in severity. Symptoms primarily result from abnormalities within the baroreceptor arc and the efferent parasympathetic and sympathetic pathways of the ANS. Generally, it is thought that cardiac parasympathetic involvement occurs early, with sympathetic innervation being preserved. This is reflected by diminished values of the Valsalva ratio and change in heart rate with deep breathing, two tests of primarily parasympathetic nerve integrity. As the disease progresses, sympathetic nerve damage is characterized by postural hypotension, sudomotor disturbances, and hypoglycemic unawareness 18 . 


\section{Conceptual Framework}

Uremia alone, or in combination with diabetes mellitus, brings about increased metabolic wastes and an increase in blood glucose levels resulting in changes to fibers within the autonomic nervous system. Specific changes that have been suggested are demyelination of preganglionic parasympathetic and sympathetic fibers, in addition to nerve lesion formation 18,101 . Another hypothesis 14,104 states that in diabetic neuropathy nerve cell hypoxia due to loss of microvasculature may result in neuronal cell degeneration and slowing of neurotransmission. There is not a consensus on the pathophysiology of dysautonomia because of the difficulty in dissecting and studying autonomic nerve fibers. Because it is much easier to examine somatic nerve fibers, much of the pathophysiologic basis of autonomic dysfunction is based on research investigating the somatic nervous system. The research findings are then applied to the autonomic nervous system. As technological improvements simplify procedures to study the autonomic nervous system ${ }^{101}$, earlier assumptions based on the somatic model are being confirmed. However, there are still many unanswered physiologic questions, such as what determines the selection and order of nerve fiber involvement and to what severity $^{21}$. A primary question, especially in light of potential interventions such as transplantation, exercise, and relaxation designed to improve autonomic function, is whether or not dysautonomia is reversible and to what extent.

Patients with ESRD experience various degrees of dysautonomia, and those with

diabetes have the greatest dysautonomia 87,93 . Early changes of dysautonomia include subtle decreases in heart rate variability due to deterioration in parasympathetic 
function 35 . In general, decreases in 24-hr frequency-domain measures (total power, LF, and $\mathrm{HF}$ ) reflect the loss of autonomic modulation earlier than either evoked tests or autonomic symptomatology $48,87,93$. This is particularly seen in the HF, which reflects parasympathetic activity as compared to the LF, which reflects primarily sympathetic activity 9,10 . Twenty-four hour HRV frequency measures were found to be diminished in studies of patients with diabetes 48,49 , patients with uremia 87,93 , and patients with uremia and diabetes $87,93,109$.

As the disease worsens, further autonomic imbalances occur, resulting in an initial increase in sympathetic activity and loss of normal sinus arrhythmia 25 . At this point, such extensive autonomic damage has occurred that maneuvers designed to evoke responses, such as a Valsalva maneuver, can no longer elicit a normal reflex. Thus, the degree of dysautonomia is such that evaluation and follow-up by evoked tests of cardiovascular autonomic function is appropriate 21 . Evoked measures, particularly the HRDB and the VR, have been used to examine persons with ESRD and diabetes since the $1960 \mathrm{~s}^{3}, 19,20,22-32$. These tests evaluate a complex reflex arc involving a stimulus, a receptor, an afferent nerve, central processing, and an efferent nerve resulting in an endorgan response ${ }^{6}$. This involves several synapses and various neurotransmitters. The tests are noninvasive, relatively simple to perform 5,28 with procedures that have been refined $^{3}, 5$, and they detect an abnormality in ESRD patients who have significant dysautonomia ${ }^{3}$. However, they are dependent on patient cooperation and they do not consistently reflect early changes in autonomic function $48,49,87,93,99$. In addition to early parasympathetic dysfunction as measured by HRDB and VR, during this time 
sympathetic function begins to deteriorate. Studies using only evoked measures are not able to detect sympathetic loss at this time, but studies using power spectral analysis are documenting the loss $49-52$.

The 24-hr time-domain measures (SDNN, SDANN, PNN50, and RMSSD) are based on standard deviations of the R-R interval. These measures are decreased with dysautonomia and, particularly the SDNN, have been associated with mortality in other populations $37-39$. However, preliminary work 87,110 found time-domain measures to be less sensitive to changes in autonomic function than either frequency-domain measures or evoked measures. The SDNN, however, was shown to hold promise for identifying an at-risk group for sudden death in the ESRD population 93 . Another timedomain measure, the SDANN, reflects circadian rhythmicity by statistically estimating the changes in heart rate due to long cycles 53,83 . A poor SDANN can result from loss of nighttime parasympathetic activity 111 , which has been found in patients with diabetes 42 . Both the PNN50 and the RMSSD are thought to be vagally mediated and, thus, reflect parasympathetic modulation 53 .

Late in the disease process, generally after 24-hr and evoked measures document dysautonomia, patients begin complaining of overt symptoms of dysautonomia 61,112 . The five primary categories of symptoms are orthostatic hypotension, sweating abnormalities, gastrointestinal problems, impotence, and hypoglycemia unawareness $18,59,61$. Usually patients present with symptoms of either orthostatic hypotension or impotence 61 . Once symptoms occur, the patient experiences severe 
dysautonomia, which can result in total autonomic failure and death unless interventions are instituted to change the downward spiral 61 .

Although the initial approach toward dysautonomia is to alleviate or control the underlying disease process, in some circumstances such as uncontrolled diabetes this is not possible. For these individuals, potentially interventions to enhance autonomic function include exercise programs, stress reduction methods such as slow deep breathing, biofeedback techniques, and medications 75,113 . Interventions for dysautonomia are just beginning to be investigated, and no studies with the ESRD population could be found. Thus, prospective studies within the ESRD population investigating potential interventions to halt further progression of dysautonomia or to reverse the signs and symptoms of dysautonomia are needed.

Figure 1 represents conceptually objective measures of autonomic function that proceed to subjective measures that assess the manifestation of dysautonomia as displayed in the center portion of the figure. Based on the assumption that actual symptoms occur late in the course of dysautonomia and that evoked measures and 24-hr HRV are hierarchical in nature, the assessment of various stages of dysautonomia is possible in ESRD patients. The left side of the figure denotes the objective measures of the autonomic nervous system, arranged based on suggested sensitivity. In addition, it includes the autonomic symptom checklist, which was developed for this series of studies to quantify subjective symptoms. The right side of the figure describes possible interventions to improve autonomic function. Interventions are based on current research reported or under study. 


\section{Measurement of Autonomic Function in Renal Disease and Diabetes}

\section{ESRD}

Metabolic derangement, increased blood glucose, ischemia, or autoimmunity lead to changes in autonomic nerve fibers including

axonal demyelination, lesion formation, and degeneration

\section{Measures Of Cardiac}

24-hr HRV frequencydomain (total power, LF, $\mathrm{HF})$

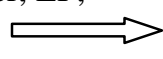

24-hr HRV time-domain (SDNN, SDANN, RMSSD)

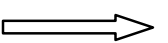

Evoked cardiovascular tests (HRDB and VR)

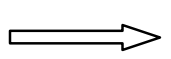

Symptom manifestation(Autonomic Symptom Checklist)
Subtle decrease in HRV with lessening parasympathetic modulation

\section{Potential Interventions}

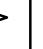

I

Loss of evoked sinus arrhythmia and decreasing sympathetic activity

Changes in circadian rhythmicity with an increasingly fixed heart rate and worsening cardiac modulation.<smiles>CCCCCCCCCC1CCCCC1</smiles>
Adaptive maneuvers to manage symptoms (ways to stand, walk, medication)

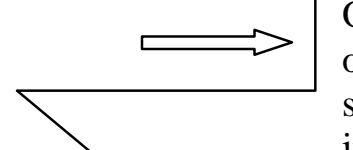

Overt symptoms including orthostatic hypotension, sweating and GI problems, impotence, and hypoglycemia unaware

Total autonomic failure potentially leading to... poten

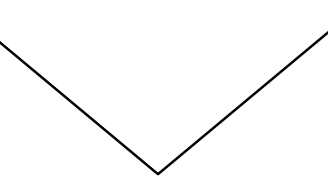

Death

Figure 1. Conceptual Framework of Dysautonomia in Uremia and Diabetes mellitus. 


\section{Chapter 2. Development and Testing of the Autonomic Symptom Checklist}

Symptomatology in early dysautonomia is often vague and nonspecific; however, dysautonomia is a common complication of end-stage renal disease and diabetes. As dysautonomia progresses it affects the function of numerous body organs resulting in a variety of signs and symptoms including orthostatic hypotension, sweating abnormalities, nocturnal diarrhea, and gastric fullness. In addition, dysautonomia can play a prominent role in impotence in men and hypoglycemia unawareness in individuals with diabetes 17 . While laboratory evoked tests and 24-hr HRV measures provide psychometrically validated evaluations of autonomic function, similarly validated instruments are not readily available for assessment of subjective symptoms of dysautonomia.

In order to document relationships among objective measures of dysautonomia and patient perceptions of their symptoms, a psychometrically validated instrument focused on patients' experiences of symptoms is needed. In 1988, the American Diabetes Association and the American Academy of Neurology recommended, in a consensus statement, ${ }^{4}$ that a validated questionnaire assessing symptoms should be part of the clinical criteria used when staging patients with diabetic polyneuropathy. Thus, the purpose of this research was to develop and test the Autonomic Symptom Checklist, an instrument capable of documenting symptoms of dysautonomia in ESRD patients with 
and without diabetes and in healthy adults. The following research questions were examined:

1. To what extent does the Autonomic Symptom Checklist demonstrate validity by distinguishing among known groups with and without dysautonomia?

2. To what extent does the Autonomic Symptom Checklist demonstrate reliability?

2a) in end-stage renal disease patients?

2b) in healthy adult individuals?

3. To what extent is the Autonomic Symptom Checklist useful as an instrument for evaluating symptoms of dysautonomia?

\section{Introduction}

A review of the genesis of dysautonomia for both ESRD patients and patients with diabetes mellitus was presented in Chapter 1. A brief summary is provided here. Dysautonomia associated with uremia has been related to increased parathyroid hormone levels, abnormalities within the baroreceptor arc, the efferent parasympathetic pathways, and reduced end-organ responsiveness to vasoconstrictor agonists such as norepinephrine ${ }^{12}$ and abnormalities in neurotransmission 101 . Diabetic autonomic neuropathies most likely result from prolonged exposure to a metabolic derangement leading to chronic, widespread neural lesions with both myelinated and unmyelinated axons being affected ${ }^{104}$. Primary factors involved are an accumulation of glucose in the peripheral nerves, a reduction of myoinositol, which is involved with lipid synthesis, changes in myelin composition and synthesis 59 , hypoxia resulting in nerve 
ischemia 14,18 , and autoimmune reactions 106,107 . Thus, individuals with ESRD with or without diabetes experience dysautonomia and data suggest that patients with ESRD and diabetes, regardless of type of diabetes, have the greatest degree of dysautonomia 87,93 .

The lack of knowledge regarding the physiological mechanisms of autonomic neuropathy partially stems from the difficulties involved in studying this part of the nervous system. Historically, the diagnosis of autonomic neuropathy was based primarily on symptoms that developed late in the disease process and were frequently nonspecific. Thus, autonomic neuropathies were thought to be uncommon in patients with diabetes 18 and ESRD 20 .

Postural hypotension is a primary symptom of dysautonomia 61 , even though it tends to be a late clinical feature 59 . It possibly indicates a lesion in the baroreceptor arc resulting in a decrease in peripheral vascular resistance ${ }^{12}$ due to a diminished response of the sympathetic vasoconstrictor fibers to the splanchnic bed, muscle and skin 18 . This prevents normal arteriolar vasoconstriction upon postural change from a sitting to a standing position. Symptoms can include dizziness, faintness, visual impairment or syncope. However, some patients are asymptomatic even when objective measures document a drop of greater than $30 \mathrm{mmHg}$ in systolic blood pressure upon standing 18 . Thus, although an objective test can measure the amount of postural hypotension, the symptoms that accompany this clinical feature are not always present.

Deficiencies in thermoregulation thought to be caused by sympathetic pathway damage result in sudomotor and vasomotor abnormalities 18 . Sudomotor, or sweating, 
abnormalities generally occur in a pattern of diminished or absent sweating in the lower extremities with compensatory increased sweating in the upper trunk and head.

Gustatory sweating, or increased sweating while eating that is unrelated to a response to spicy foods, is a less common symptom 59 . Vasomotor abnormalities present vaguer symptoms of thermoregulation dysfunction than sudomotor abnormalities. In general, vasomotor complaints center around the individual's feet being either too cold or too hot. These symptoms are thought to be caused by a failure to reflexively vasodilate or vasoconstrict in response to body temperature changes 18 .

Hosking et al. ${ }^{59}$ reported that even when the entire gastrointestinal system is involved with generalized hypotonic or hypertonic activity, the patient is often asymptomatic. The genesis of gastrointestinal dysfunctions, which can range from esophageal atony to diarrhea, involves damage to the parasympathetic and sympathetic nervous system 18 , in addition to possible autoimmune factors 62 . Diarrhea is the most prevalent symptom within this category 59 . It is fairly characteristic and can last from hours to days, with liquid stooling up to 20 or more times a day. Diarrhea at night and fecal incontinence are common. Constipation is also a frequent complaint of patients with dysautonomia, but it is unknown how this is related to the autonomic nervous system ${ }^{18}$, or if it is related to other factors such as medications the patient is taking. Symptoms of urogenital system involvement primarily include changes in the ability to control the bladder and impotence 61 . Symptoms of deficient bladder control develop insidiously, progress slowly, and are complex to differentiate from common complications such as a bladder infection. This makes it difficult to evaluate urinary changes as a symptom of dysautonomia. Damage to the parasympathetic nervous system 
causes impotence with loss of penile erection in diabetic men with a prevalence of up to $50 \% 18,59,60$. Data on female sexual function is scant. Other urogenital symptoms that can occur include ejaculation failure and loss of testicular pain to pressure. Both of these symptoms are believed to result from widespread sympathetic denervation to the pelvic $\operatorname{area}^{18}$. Impotence is not always included as a diagnostic feature ${ }^{63}$ because of confounding variables, such as psychogenic factors or the influence of age and mediations ${ }^{59}$, which can affect penile erection.

Hypoglycemia unawareness is the failure of the patient to recognize symptoms of low blood sugar. This involves both parasympathetic and sympathetic dysfunction, however, the symptoms associated with hypoglycemia, including tachycardia and headache, are sympathetic in origin 18 . Thus, hypoglycemia unawareness indicates the progression of autonomic dysfunction from parasympathetic to sympathetic involvement. It is considered a late symptom manifestation of diabetic autonomic neuropathy and can be particularly devastating resulting in coma and death.

In the 1970s, Ewing and Winney 20 began using bedside autonomic function tests to aid in interpretation of these vague symptoms; thus, the diagnosis of autonomic neuropathies became more objective leading to increased recognition and treatment of dysautonomia at an earlier stage. However, the treatment of dysautonomia is not definitive and the progression of dysautonomia still results in symptomatology. Presently, there are valid and reliable objective, physiologic measures of dysautonomia 21,53 however, a valid and reliable instrument to measure symptomatology is not available. For research purposes, a self-administered autonomic symptomatology instrument could be used to identify when in the progression of 
dysautonomia symptoms occur. Thus, the purpose of this investigation was to develop and test an instrument designed to measure symptoms of dysautonomia.

\section{Methodology}

\section{Design}

A descriptive, cross-sectional design was used to examine the symptomatology of dysautonomia experienced by ESRD patients being evaluated for a kidney or kidneypancreas transplant. In addition, healthy individuals were recruited as controls. Based on previously published data suggesting that subjects with ESRD and diabetes have poorer

autonomic function than ESRD without diabetes 87,93 , three known groups with varying levels of autonomic function were identified. Validity was then determined by comparing the autonomic function of these known groups; healthy controls, patients with ESERD and diabetes mellitus (ESRD DM) and patients with ESRD without diabetes mellitus (ESRD NonDM). A correlational design using test-retest data was used to establish reliability of the Autonomic Symptom Checklist (ASC).

\section{Sample}

A convenience sample of 242 pre kidney or kidney-pancreas transplant patients from a major transplantation center in the southern United States and 34 healthy controls were enrolled. All pretransplant subjects had ESRD, with or without diabetes, which was severe enough to warrant referral for transplantation. The healthy controls were recruited from a group of students, faculty, and friends. Each healthy control was asked a series of questions to evaluate for potential dysautonomia and excluded if any was suspected. Inclusion criteria for both ESRD patients and healthy controls included being over 18 
years of age. Patients and healthy controls were excluded if they were pregnant or found to have a co-existing neurological deficit that altered autonomic function such as spinal cord injury, peripheral nerve trauma, and degenerative disease of the spinal cord.

Previously published data 87,93 documented the level of dysautonomia in the ESRD group allowing stratification into three groups: healthy controls (Control, $n=34)$, ESRD patients without diabetes mellitus (NonDM, n=159), and ESRD subjects with diabetes mellitus (DM, n=85).

A subgroup of control $(n=15)$ and ESRD patients $(n=7)$ participated in the testretest portion of the study. The control subgroup was a convenience sample of those available to retake the test within two to seven weeks (mean=4.5 weeks) of the original testing. The seven ESRD patients were a convenience sample chosen from a patient group that was available by telephone during a 4-day period that corresponded to three to five weeks (mean=5.5 weeks) from the original testing. Because no change was observed in objective measures of autonomic function over a two to 10 week period 46 , it was thought that retesting within 5 weeks would result in scores minimally influenced by changes in autonomic function.

The control, NonDM and DM groups (see Table 2) had no significant gender difference and were predominately men $(54 \%, 64 \%$ and $58 \%$, respectively). In addition, there was no significant difference in mean age (Control=42 yrs., NonDM=42 yrs., and $\mathrm{DM}=44$ yrs., respectively). The NonDM group had a significantly higher $(\mathrm{p} \leq 0.05)$ percentage of African-Americans (68\%) as compared to the DM group (42\%) and control group (9\%). Previous work has suggested an inverse relationship between autonomic function and age $27,64,65,114$ with a lack of consensus on the relationship between 
autonomic function and gender 27,31 . No reports on the relationship between autonomic function and race could be found. Subgroups consisting of only men from the control, NonDM, and DM groups were used to establish the validity of the Impotence Category. There was no significant difference in mean age (see Table 3) among the men subgroups (control=39 yrs., NonDM=42 yrs., DM=46 yrs.), however, the NonDM group had a significantly higher $(\mathrm{p} \leq 0.05)$ percentage of African-Americans $(68 \%)$ as compared to the DM $(50 \%)$ and the control groups $(0 \%)$.

Healthy control $(n=15)$ and ESRD patient $(n=7)$ subgroups were identified to establish reliability of the ASC. There was no significant difference in mean age (see Table 4) among the subgroups (control=41 yrs., ESRD=47 yrs.), however, the ESRD subgroup had a significantly higher $(\mathrm{p} \leq 0.05)$ percentage of African-Americans $(57 \%)$ as compared to the control group (13\%).

Displayed in Table 5 are the characteristics of the ESRD DM group that were divided into those aware of symptoms of hypoglycemia and those unaware of the symptoms. The Unaware group had more women (59\% vs. 36\%) and had a slightly younger mean age (40 yrs. vs. 45 yrs.). They were similar on race with the Unaware group being 34\% African-Americans and the Aware group being $41 \%$. 
Table 2. Characteristics of Healthy Controls, ESRD Patients with Diabetes Mellitus (DM), ESRD Patients Without Diabetes Mellitus (NonDM), and the Total Sample

\begin{tabular}{|c|c|c|c|c|}
\hline & $\begin{array}{l}\text { Healthy } \\
\text { Controls } \\
(n=34)\end{array}$ & $\begin{array}{l}\text { ESRD with DM } \\
\qquad(\mathrm{n}=85)\end{array}$ & $\begin{array}{l}\text { ESRD NonDM } \\
\qquad(\mathrm{n}=159)\end{array}$ & $\begin{array}{l}\text { Total Sample } \\
\qquad(\mathrm{n}=276)\end{array}$ \\
\hline Age (mean, SD) & $42 \pm 9.1$ & $44 \pm 10.4$ & $42 \pm 12.1$ & $42 \pm 11.3$ \\
\hline Range (yrs.) & $25-65$ & $25-67$ & $17-69$ & $17-69$ \\
\hline \multicolumn{5}{|l|}{ Gender (n/\%) } \\
\hline Women & $16(46 \%)$ & $36(42 \%)$ & $57(36 \%)$ & $109(40 \%)$ \\
\hline Men & $19(54 \%)$ & $49(58 \%)$ & $102(64 \%)$ & $170(60 \%)$ \\
\hline \multicolumn{5}{|l|}{ Race (n/\%) } \\
\hline African-Amer. & $3(9 \%)^{*}$ & $35(42 \%)^{*}$ & $108(68 \%)^{*}$ & $146(53 \%)$ \\
\hline Caucasian & $31(89 \%)^{*}$ & $48(57 \%)^{*}$ & $50(32 \%)^{*}$ & $129(46 \%)$ \\
\hline Other & $1(2 \%)$ & $1(1 \%)$ & $0(0 \%)$ & $2(1 \%)$ \\
\hline
\end{tabular}

Note. ${ }^{*}=\mathrm{p} \leq 0.05$ for groups having like symbols; ESRD=end-stage renal disease. 
Table 3. Characteristics of Male Subgroups in the Healthy Controls, ESRD Patients with Diabetes Mellitus (DM), ESRD Patients Without Diabetes Mellitus (NonDM), and the Total Sample

\begin{tabular}{lcccc}
\hline & $\begin{array}{c}\text { Healthy Controls } \\
(\mathrm{n}=19)\end{array}$ & $\begin{array}{c}\text { ESRD with DM } \\
(\mathrm{n}=48)\end{array}$ & $\begin{array}{c}\text { ESRD NonDM } \\
(\mathrm{n}=102)\end{array}$ & $\begin{array}{c}\text { Total Sample } \\
(\mathrm{n}=169)\end{array}$ \\
\hline Age (mean, SD) & $39 \pm 10.0$ & $46 \pm 9.8$ & $42 \pm 12.1$ & $43 \pm 114$ \\
Range (yrs.) & $25-65$ & $30-67$ & $18-69$ & $18-69$ \\
Race (n/\%) & $0(0 \%)^{*}$ & $24(50 \%)^{*}$ & $68(67 \%)^{*}$ & $92(54 \%)$ \\
African-Amer. & $18(94 \%)^{*}$ & $24(50 \%)^{*}$ & $34(33 \%)^{*}$ & $76(45 \%)$ \\
Caucasian & $1(6 \%)$ & $0(0 \%)$ & $0(0 \%)$ & $1(1 \%)$ \\
Other & & & & \\
\hline
\end{tabular}

Note. ${ }^{*}=\mathrm{p} \leq 0.05$ for groups having like symbols; ESRD=end-stage renal disease. 
Table 4. Characteristics of Healthy Control and ESRD Patient Reliability Subgroups

\begin{tabular}{lcc}
\hline & $\begin{array}{c}\text { Healthy Controls } \\
(\mathrm{n}=15)\end{array}$ & $\begin{array}{c}\text { ESRD Patients } \\
(\mathrm{n}=7)\end{array}$ \\
\hline Age (mean, SD) & $41 \pm 7.8$ & $47 \pm 17.9$ \\
Range & $25-55$ & $19-67$ \\
Gender (n/\%) & $8(53 \%)$ & $4(57 \%)$ \\
Women & $7(47 \%)$ & $3(43 \%)$ \\
Men & & \\
Race (n/\%) & $2(13 \%)^{*}$ & $4(57 \%)^{*}$ \\
African-American & $13(87 \%)^{*}$ & $3(43 \%)^{*}$ \\
Caucasian & & \\
\hline
\end{tabular}

Note. ${ }^{*}=\mathrm{p} \leq 0.05$ for groups having like symbols; ESRD=end-stage renal disease 
Table 5. Characteristics of Hypoglycemia Unaware and Hypoglycemia Aware Subgroups.

\begin{tabular}{lcc}
\hline & $\begin{array}{c}\text { Hypoglycemia Unaware } \\
(\mathrm{n}=31)\end{array}$ & $\begin{array}{c}\text { Hypoglycemia Aware } \\
(\mathrm{n}=34)\end{array}$ \\
\hline Age (mean,SD) & $40 \pm 8.8$ & $45 \pm 10.9$ \\
Range & $25-64$ & $26-67$ \\
Gender (n/\%) & $18(59 \%)$ & $14(36 \%)$ \\
Women & $13(41 \%)$ & $25(64 \%)$ \\
Men & $11(34 \%)$ & $16(41 \%)$ \\
Race (n/\%) & $21(66 \%)$ & $22(56 \%)$ \\
African-American & 0 & $1(3 \%)$ \\
Caucasian & & \\
Other & & \\
\hline
\end{tabular}

Note. No significant differences were found among groups. 


\section{Instrument}

The aims of the ASC were to recognize the perceived presence, distribution and severity of autonomic symptoms. Initially, the instrument was compiled using information from several expert sources $59,61,115$. Subsequently, a focus group met over a five-week period for four, two hour sessions to refine the questions and simplify the reading level of the instrument. The focus group was comprised of people with experience as transplant coordinators, diabetic educators, and researchers in addition, one person had type 2 diabetes mellitus. Following focus group discussions, the ASC was pilot tested on four ESRD patients and further revisions were made. Revisions included clarifying terminology and adding a time-frame to the directions.

The revised ASC was developed as a self-administered checklist that assessed dysautonomia symptoms in five categories with 32-items each with a 7-point Likert scale $^{116}$ (see Appendix C). A self-administered format was chosen because, typically, the individual's perception of these symptoms is what alerts the provider to the possibility of dysautonomia. Three categories, Orthostatic Hypotension, Sudomotor Abnormalities, and Gastrointestinal Problems, are to be completed by all respondents, while two categories are also completed by subgroups: the Impotence category is to be completed by men; and the Hypoglycemia Unaware is to be completed by individuals with diabetes mellitus.

Each of the five categories consists of four to six questions, each answered using a seven-point Likert scale with 0 being "never" and 6 being "always". The individual is asked to circle the number that indicates the severity of that symptom. The last question in each category is classified as an "impact" question and used to obtain a score reflecting 
patients' perceptions of how problematic that category of symptoms is for them. This question was included because the presence of a symptom and the degree to which a symptom creates problems are not always the same. In addition, information is obtained concerning each patient's current medications, alcohol and caffeine intake, and tobacco use. These products could affect function of the autonomic nervous system ${ }^{21}$, but it is unknown as to what extent.

The Orthostatic Hypotension category consists of five questions (30 points possible) focused on symptoms of hypotension upon standing. These symptoms include rapid heartbeat, blurred vision, an upset stomach, dizziness, and clammy skin. The Sudomotor Abnormalities category consists of three questions (18 points possible) focused on an increase or decrease in sweating. The Gastrointestinal Problems category consists of five questions (30 points possible) focusing on bowel and stomach disorders. The Impotence category consists of four questions (24 points possible) focused on the ability to have and maintain an erection. The Hypoglycemia Unaware category consists of four questions (24 points possible) focused on subjects' ability to recognize symptoms associated with a low blood sugar. Each category of symptom questions is followed by an impact question "how much of a problem are these symptoms for you?" (6 points possible).

Patient responses are entered into the database as scored by the individual, except for questions $4 \mathrm{a}, 4 \mathrm{~b}$, and $4 \mathrm{c}$ in the Impotence category and question $5 \mathrm{a}$ in the Hypoglycemia Unawareness category, which are written in reverse fashion and, therefore, scored in reverse fashion. Category and index calculations are performed 
based on these values with higher scores indicating greater symptoms. See Appendix D for definitions and a detailed description of the calculations.

For each patient, two scores per category are reported: symptom score and impact score. The category symptom score was calculated by obtaining a raw score for each category then dividing the raw score by the number of questions in that category. Each category symptom score has a range from $0-6$. The category impact score is obtained directly from the one impact question and also ranged from $0-6$. Each category symptom score and impact score can be compared across groups.

Two other scores reported are total symptom score and total impact score. The total symptom score (6 points possible) are the sum of category symptom scores divided by the number of categories reported (e.g., 5 for DM women, 6 for DM men). The total impact score (6 points possible) is calculated by dividing the total number of points scored on the category impact questions by the number of impact questions possible for that group. Both the total symptom score and the total impact score can be compared across groups.

The ASC index score (36 points possible) is a single measure designed to reflect the patient's perception of experienced dysautonomia symptoms and the impact of those symptoms on the patient. It is calculated by multiplying the total symptom score and the total impact score and can be compared across groups.

\section{Procedure}

Subjects with ESRD were recruited from a group of individuals being examined for dysautonomia as part of their transplantation evaluation at the University of Tennessee, Memphis. At the time of evaluation the study was explained to potential 
subjects, eligibility was ascertained, and written consent obtained (see Appendix B).

Baseline data including health history and demographic data were also obtained prior to administration of the ASC. The subject was asked to return the ASC with other questionnaires they completed as part of their evaluation for transplantation.

The normal healthy controls were recruited from a group of students, faculty, and friends at the University of Tennessee, Memphis. Thirty-one of the healthy controls had participated in previous autonomic function testing which documented normal autonomic function. For the controls with no prior autonomic function testing, demographic data and follow-up questions from the investigator were designed to screen for physical problems indicating potential dysautonomia.

A subgroup from both the control group and the ESRD group were identified and asked to complete the ASC a second time. The control subgroup ( $n=15)$ consisted of the controls who had taken the initial test two to five weeks previously. All who qualified completed the retake. The patient subgroup $(\mathrm{n}=7)$ consisted of patients who had initially completed the ASC two to six weeks previously. The ASC was re-administered by telephone and the first seven subjects contacted by phone comprised the subgroup. The two to six week period between the pre and posttest allowed enough time to prevent recall 116 , yet not enough time for significant change in health status to occur which could alter symptomatology 46 .

\section{Data Analysis}

Content validity of the ASC was ascertained by deriving the questions in the ASC from published works from known experts in autonomic dysfunction and diabetes. In addition, a group of experts participated in a focus group to review and refine the 
questions. Univariate analysis was used to compare the equality of the groups.

Construct validity was ascertained using ANOVA tests to compare the means of the three known groups for three of the categories (Orthostatic Hypotension, Sudomotor Symptoms, Gastrointestinal Symptoms). An ANOVA test on men in each of the three groups was used to obtain a measure of validity of the Impotence Category. Validity of Hypoglycemia Unaware was assessed using Student's t-test. Patients reported to be aware of symptoms of hypoglycemia were placed in one group; patients reported to be unaware of symptoms were placed in a second group. This was based on information gathered by a clinician assessing the patients' awareness of hypoglycemia through a probing interview of the patient and family. Correlational analysis was used to evaluate reliability of the ASC. Test-retest data were obtained on a subset of both controls and subjects to obtain a coefficient of stability.

\section{Results}

\section{Validity}

The focus group concurred that the refined ASC had content validity. Construct validity testing resulted with the DM group reporting the greatest symptomatology, followed by the NonDM group, and with least symptoms, the control group. This pattern was present for all symptom categories. There was significant difference $(p \leq 0.0001)$ among all groups for three symptom scores (orthostatic hypotension, gastrointestinal problems, and impotence) and two impact scores (gastrointestinal problems and impotence). There was significant difference $(\mathrm{p} \leq 0.003)$ between healthy controls and both ESRD groups (see Table 6) for one symptom score (sudomotor abnormalities) and 
two impact scores (orthostatic hypotension and sudomotor abnormalities). In addition, findings demonstrated a significant difference $(\mathrm{p} \leq 0.03)$ in symptoms between patients with diabetes who were hypoglycemia aware and hypoglycemia unaware (Table 7). Thus, the ASC differentiated among groups with varying levels of dysautonomia and identified a group with increased symptoms.

\section{Reliability}

A coefficient of stability over a two to five week period was measured with a testretest technique using Pearson correlation ${ }^{116}$ (see Tables 8 and 9). Within the control subgroup, symptom score and impact score reliability coefficients ranged from 0.69 (Gastrointestinal Problems) to 0.87 (Orthostatic Hypotension) with $\mathrm{p} \leq 0.004$. Within the patient subgroup, the symptom score reliability coefficients ranged from $0.59, \mathrm{p} \leq 0.03$ (Sudomotor Abnormalities) to 1.00, $\mathrm{p} \leq 0.000$ (Hypoglycemia Unaware). Because the $\mathrm{r}$ values ranged between $0.59-0.87$, there was a moderate to high positive correlation and

consistency between the two tests 116 . However, the reliability coefficient for the Sudomotor Impact Score was low for patients $(\mathrm{r}=0.05, \mathrm{p} \leq 0.9)$.

\section{Other findings}

Mean symptom and impact scores for controls, ESRD NonDM and ESRD DM groups were reported by gender in Table 10. Table 11 displays data ranked from highest score (most symptom or problem) to lowest score (least symptom or problem). All ESRD patient groups when examined by gender have the same symptom and impact score ranking: Impotence Category, Hypoglycemia Awareness, Gastrointestinal Problems, Orthostatic Hypotension, and Sudomotor Disorders. 
Table 6. Symptom and Impact Scores Comparing Healthy Controls, Patients with ESRD and Patients with both ESRD and DM on Four Sections of the Autonomic Symptom Checklist

\begin{tabular}{|c|c|c|c|c|c|c|c|c|}
\hline & \multicolumn{2}{|c|}{$\begin{array}{c}\text { Orthostatic } \\
\text { Hypotension }\end{array}$} & \multicolumn{2}{|c|}{$\begin{array}{c}\text { Sudomotor } \\
\text { Abnormalities }\end{array}$} & \multicolumn{2}{|c|}{$\begin{array}{l}\text { Gastrointestinal } \\
\text { Problems }\end{array}$} & \multicolumn{2}{|c|}{ Impotence } \\
\hline & $\begin{array}{l}\text { Symptom } \\
\text { Score }\end{array}$ & $\begin{array}{c}\text { Symptom } \\
\text { Impact Score }\end{array}$ & $\begin{array}{l}\text { Symptom } \\
\text { Score }\end{array}$ & $\begin{array}{c}\text { Symptom } \\
\text { Impact } \\
\text { Score }\end{array}$ & $\begin{array}{l}\text { Symptom } \\
\text { Score }\end{array}$ & $\begin{array}{c}\text { Symptom } \\
\text { Impact Score }\end{array}$ & Symptom Score & $\begin{array}{c}\text { Symptom } \\
\text { Impact Score }\end{array}$ \\
\hline $\mathrm{n}$ & 279 & 272 & 279 & 260 & 279 & 262 & 159 & 151 \\
\hline $\begin{array}{l}\text { Range of } \\
\text { possible scores }\end{array}$ & $0-6$ & $0-6$ & $0-6$ & $0-6$ & $0-6$ & $0-6$ & $0-6$ & $0-6$ \\
\hline GROUP & & & & & & & & \\
\hline $\begin{array}{l}\text { Control }(n=35) \\
(n=19 ; \text { Impotence })\end{array}$ & $0.20 \pm 0.41 *$ & $0.17 \pm 0.5^{* \dagger}$ & $0.44 \pm 0.8^{*}$ & $0.26 \pm 0.7 *$ & $0.40 \pm 0.5^{*}$ & $0.34 \pm 0.73 *$ & $0.71 \pm 1.1^{*}$ & $0.50 \pm 1.2 *$ \\
\hline $\begin{array}{l}\text { NonDM }(n=159) \\
(n=102 ; \text { Impotence })\end{array}$ & $0.89 \pm 1.01 *$ & $1.15 \pm 1.5 \dagger$ & $0.75 \pm 1.1 \dagger$ & $0.67 \pm 1.3 \dagger$ & $1.22 \pm 1.1 *$ & $1.53 \pm 1.7 *$ & $2.53 \pm 1.6^{*}$ & $2.35 \pm 2.3 *$ \\
\hline $\mathrm{P}$ value $<$ & 0.0001 & 0.0001 & 0.0008 & 0.003 & 0.0001 & 0.0001 & 0.0001 & 0.0001 \\
\hline
\end{tabular}

Note. * and $\dagger$ indicate significance for groups having like symbols. All values are given as means \pm SD. ESRD=end-stage renal disease, DM=diabetes mellitus, NonDM=nondiabetes mellitus. 
Table 7. Symptom and Impact Scores from the Autonomic Symptom Checklist Which Compares Hypoglycemia Aware and Unaware ESRD DM Patients

\begin{tabular}{|c|c|c|}
\hline & \multicolumn{2}{|c|}{$\begin{array}{c}\text { Hypoglycemia } \\
\text { Unaware } \\
\text { (24 points possible) }\end{array}$} \\
\hline Range of scores & $\begin{array}{l}\text { Symptom } \\
\text { Score } \\
0-6\end{array}$ & $\begin{array}{c}\text { Symptom Impact } \\
\text { Score } \\
0-6\end{array}$ \\
\hline GROUP & & \\
\hline Not aware $(n=31)$ & $2.40 \pm 1.0 *$ & $3.61 \pm 2.1 *$ \\
\hline Aware $(n=34)$ & $1.61 \pm 1.0 *$ & $2.48 \pm 2.0^{*}$ \\
\hline $\mathrm{P}$ value $<$ & 0.002 & 0.03 \\
\hline
\end{tabular}


Table 8. Correlations for Pre- and Posttest ASC Scores for Three Symptom Categories

\begin{tabular}{lcccccc}
\hline & \multicolumn{2}{c}{$\begin{array}{c}\text { Orthostatic } \\
\text { Hypotension }\end{array}$} & $\begin{array}{c}\text { Sudomotor } \\
\text { Symptoms }\end{array}$ & $\begin{array}{c}\text { Gastrointestinal } \\
\text { Symptoms }\end{array}$ \\
& $\begin{array}{c}\text { Symptom } \\
\text { Score }\end{array}$ & $\begin{array}{c}\text { Impact } \\
\text { Score }\end{array}$ & $\begin{array}{c}\text { Symptom } \\
\text { Score }\end{array}$ & $\begin{array}{c}\text { Impact } \\
\text { Score }\end{array}$ & $\begin{array}{c}\text { Symptom } \\
\text { Score }\end{array}$ & $\begin{array}{c}\text { Impact } \\
\text { Score }\end{array}$ \\
$\begin{array}{l}\text { ESRD Sample } \\
\text { (n=7) }\end{array}$ & 0.79 & 0.78 & 0.59 & 0.05 & 0.72 & 0.91 \\
$\begin{array}{l}\text { Healthy Controls } \\
(\mathrm{n}=15)\end{array}$ & 0.87 & 1.00 & 0.81 & 1.00 & 0.69 & 0.84 \\
\hline
\end{tabular}

Note. Values represent correlation coefficients.

Table 9. Correlations for Pre- and Posttest ASC Scores for Impotence and Hypoglycemia Categories.

\begin{tabular}{ccccc}
\hline & \multicolumn{2}{c}{$\begin{array}{c}\text { Impotence } \\
\text { (Patients \& Controls) }\end{array}$} & $\begin{array}{c}\text { Hypoglycemia Unaware } \\
\text { (Diabetics only) }\end{array}$ \\
\hline & Symptom & Impact & Symptom & Impact Score \\
& Score & Score & Score & \\
r values & 10 & 10 & 2 & 2 \\
& 0.99 & 0.76 & 1.00 & Not calculated \\
\hline
\end{tabular}

Note. Values represent correlation coefficients. 
Because pretransplant kidney and kidney-pancreas patients have poorer objective measures of autonomic function when compared to posttransplant patients, we decided, in addition to the research questions, to examine the validity of the ASC by administering it to posttransplant patients. The ASC was given to independent samples of pretransplant $(\mathrm{n}=233)$ and posttransplant patients ( $\mathrm{n}=77,6-12$ months posttransplant). The samples were similar in diabetic status, age, and gender (see Table 12). Hypoglycemia data are not reported because all but 7 of the 29 patients with diabetes received a pancreas transplant and no longer experienced hypoglycemia. Posttransplant patients reported statistically significant $(\mathrm{p} \leq 0.05)$ fewer symptoms in two categories (Orthostatic Hypotension and Gastrointestinal Problems) with essentially no difference in sudomotor symptoms. Index Score, which reflects both symptoms and impact of the symptoms, was significantly $(\mathrm{p} \leq 0.05)$ better for the posttransplant group.

In addition, a subset of patients with both pre and posttransplant evaluations was identified ( $\mathrm{n}=19)$, which was similar to the larger posttransplant sample in terms of diabetic status, gender, and type of transplant (64\% kidney only). Although significance was not obtained in matched patients (see Table 13), symptom improvement was seen in the majority of individuals in this subset for Orthostatic Hypotension (10 of 19), Gastrointestinal Problem (10 of 19), and Index Score (12 of 19). Thus, the ASC documents a decrease in symptoms of dysautonomia following kidney and kidneypancreas transplant. 
Table 10. ASC Mean Symptom and Impact Scores by Group and Gender

\begin{tabular}{|c|c|c|c|c|c|c|}
\hline & \multicolumn{2}{|c|}{ Healthy Controls } & \multicolumn{2}{|c|}{ ESRD NonDM } & \multicolumn{2}{|c|}{$E S R D D M$} \\
\hline & $\begin{array}{l}\text { Women } \\
(\mathrm{n}=16)\end{array}$ & $\begin{array}{c}\text { Men } \\
(n=18)\end{array}$ & $\begin{array}{l}\text { Women } \\
(\mathrm{n}=57)\end{array}$ & $\begin{array}{c}\text { Men } \\
(n=102)\end{array}$ & $\begin{array}{l}\text { Women } \\
(n=36)\end{array}$ & $\begin{array}{c}\text { Men } \\
(n=49)\end{array}$ \\
\hline Symptom Scores & & & & & & \\
\hline $\begin{array}{l}\text { Orthostatic } \\
\text { Hypotension }\end{array}$ & 0.26 & 0.14 & 0.86 & 0.91 & 1.38 & 0.92 \\
\hline $\begin{array}{l}\text { Sudomotor } \\
\text { Abnormalities }\end{array}$ & 0.54 & 0.35 & 0.76 & 0.74 & 1.27 & 0.75 \\
\hline $\begin{array}{l}\text { Gastrointestinal } \\
\text { Problem }\end{array}$ & 0.41 & 0.56 & 1.24 & 1.21 & 1.95 & 1.22 \\
\hline Impotence & na & 0.71 & na & 2.53 & na & 2.53 \\
\hline $\begin{array}{l}\text { Hypoglycemia } \\
\text { Unawareness }\end{array}$ & na & na & na & na & 2.12 & 2.00 \\
\hline Impact Scores & & & & & & \\
\hline $\begin{array}{l}\text { Orthostatic } \\
\text { Hypotension }\end{array}$ & 0.38 & 0.00 & 0.99 & 1.24 & 1.89 & 1.42 \\
\hline $\begin{array}{l}\text { Sudomotor } \\
\text { Abnormalities }\end{array}$ & 0.44 & 0.11 & 0.73 & 0.64 & 1.20 & 1.09 \\
\hline $\begin{array}{l}\text { Gastrointestinal } \\
\text { Problem }\end{array}$ & 0.38 & 0.32 & 1.57 & 1.52 & 2.48 & 2.10 \\
\hline Impotence & na & 0.5 & na & 2.35 & na & 3.94 \\
\hline $\begin{array}{l}\text { Hypoglycemia } \\
\text { Unawareness }\end{array}$ & na & na & na & na & 2.94 & 2.96 \\
\hline
\end{tabular}

gु Note. ESRD=end-stage renal disease; NonDM=nondiabetic; $\mathrm{DM}=$ diabetes mellitus; na=not applicable. 
Table 11. Highest to Lowest ASC Mean Symptom and Impact Scores for ESRD Patients by Group and Gender

\begin{tabular}{|c|c|c|c|c|c|c|c|c|}
\hline & \multicolumn{3}{|c|}{ Symptom Score } & & \multicolumn{4}{|c|}{ Impact Score } \\
\hline & \multicolumn{2}{|c|}{ Men } & \multicolumn{2}{|c|}{ Women } & \multicolumn{2}{|c|}{ Men } & \multicolumn{2}{|c|}{ Women } \\
\hline & DM & NonDM & DM & NonDM & $\mathrm{DM}$ & NonDM & DM & NonDM \\
\hline Impotence & 2.53 & 2.53 & na & na & 3.94 & 2.35 & na & na \\
\hline $\begin{array}{l}\text { Hypoglycemia } \\
\text { Unaware }\end{array}$ & 2.00 & na & 2.12 & na & 2.96 & na & 2.94 & na \\
\hline $\begin{array}{l}\text { Gastrointestinal } \\
\text { Problems }\end{array}$ & 1.22 & 1.21 & 1.95 & 1.24 & 2.10 & 1.52 & 2.48 & 1.57 \\
\hline $\begin{array}{l}\text { Orthostatic } \\
\text { Hypotension }\end{array}$ & 0.92 & 0.91 & 1.38 & 0.86 & 1.42 & 1.24 & 1.89 & 0.99 \\
\hline Sudomotor Disorders & 0.75 & 0.74 & 1.27 & 0.76 & 1.09 & 0.64 & 1.20 & 0.73 \\
\hline
\end{tabular}

Note. ESRD=end-stage renal disease; NonDM=nondiabetic; DM=diabetes mellitus; na=not applicable.

d 
Table 12. Characteristics of Independent Samples and Matched Samples of Pre and Posttransplant ESRD Patients

\begin{tabular}{|c|c|c|c|c|c|}
\hline & \multicolumn{4}{|c|}{ NonMatched } & \multirow{2}{*}{$\frac{\text { Matched }}{\text { Pre and PostTransplant }}$} \\
\hline & \multirow[t]{2}{*}{ PreTransplant } & \multicolumn{3}{|c|}{ PostTransplant } & \\
\hline & & Total Group & 6 mo. PostTx & 12 mo. PostTx & \\
\hline $\mathrm{n}$ & 233 & 68 & 37 & 31 & 19 \\
\hline Age & $42.5 \pm 11.6$ & $41.3 \pm 11.1$ & $41.2 \pm 10.8$ & $41.5 \pm 11.4$ & $40.2 \pm 12.4$ \\
\hline \multicolumn{6}{|l|}{ PreTx DM Status } \\
\hline $\mathrm{DM}$ & $85(36 \%)$ & $29(43 \%)$ & $15(40 \%)$ & $14(45 \%)$ & $8(42 \%)$ \\
\hline NonDM & $148(64 \%)$ & $39(57 \%)$ & $22(60 \%)$ & $17(55 \%)$ & $11(58 \%)$ \\
\hline \multicolumn{6}{|l|}{ Gender } \\
\hline Men & $140(60 \%)$ & $44(65 \%)$ & $24(65 \%)$ & $20(65 \%)$ & $11(58 \%)$ \\
\hline Women & $93(40 \%)$ & $24(35 \%)$ & $13(35 \%)$ & $11(35 \%)$ & $8(42 \%)$ \\
\hline \multicolumn{6}{|l|}{ Race } \\
\hline African-Am. & $134(58 \%)$ & $19(28 \%)$ & $10(27 \%)$ & $9(29 \%)$ & $4(21 \%)$ \\
\hline Caucasian & $96(41 \%)$ & $49(72 \%)$ & $27(73 \%)$ & $22(71 \%)$ & $15(79 \%)$ \\
\hline Other & $1(1 \%)$ & & & & \\
\hline \multicolumn{6}{|l|}{ Type of Tx } \\
\hline Kidney alone & $186(80 \%)$ & $46(68 \%)$ & $24(64 \%)$ & $22(70 \%)$ & $12(64 \%)$ \\
\hline Pancreas or KP & $47(20 \%)$ & $22(32 \%)$ & $13(36 \%)$ & $9(30 \%)$ & $7(36 \%)$ \\
\hline
\end{tabular}

Note. ESRD=end-stage renal disease; NonDM=nondiabetic; DM=diabetes mellitus; Tx=transplant; KP=kidney-pancreas. 
Table 13. ASC Category Mean Symptom Scores and Index Scores for Independent and Matched Samples of Pre and Posttransplant ESRD Patients

\section{Category Mean Symptom Scores}

\begin{tabular}{|c|c|c|c|c|c|}
\hline Group & Orthostatic & Sudomotor & GI & Impotence & Index \\
\hline \multicolumn{6}{|l|}{ Independent Samples } \\
\hline $\operatorname{PreTx}(n=233)$ & $0.99 *$ & 0.93 & $1.36^{*}$ & 2.98 & $3.03^{*}$ \\
\hline PostTx (n=93) & $0.72 *$ & 0.92 & $1.04 *$ & 2.93 & $1.65^{*}$ \\
\hline \multicolumn{6}{|l|}{ Matched } \\
\hline $\operatorname{PreTx}(n=19)$ & 0.82 & 0.91 & 1.47 & 1.91 & 2.50 \\
\hline PostTx $(n=19)$ & 0.52 & 0.56 & 1.11 & 2.67 & 1.32 \\
\hline
\end{tabular}




\section{Discussion}

\section{Validity}

Content validity was established by an expert panel. Construct validity was established by the ability of the Autonomic Symptom Checklist to significantly differentiate among three groups with known dysautonomia. Both the ESRD patients with diabetes and those without diabetes were different from the control group and from each other on orthostatic hypotension, gastrointestinal problems and impotence symptom scores. The sudomotor symptom score differentiated between healthy controls and patients with ESRD and diabetes. In addition, the groups with diabetes, which have been shown to have the poorest evoked cardiac measures and 24-hr HRV measures of autonomic function 87 , also have the highest symptom scores on all subscales. Thus, increased symptomatology was seen in patients with worsening physiologic autonomic function. In addition, when the ASC was administered to pre and posttransplant kidney and pancreas-kidney patients, the ASC documented increased symptomatology in the pretransplant group, which is known to have poorer objective measures of dysautonomia 41,99 , as compared to the posttransplant group. This finding documents the ability of the ASC to monitor changes in autonomic function and, thus, supports its clinical utility in longitudinal research protocols. In addition, it shows that in a small group of kidney and kidney-pancreas posttransplant patients, symptomatology decreased by 6 to 12 months after transplant.

Age and gender have been identified as the primary factors, outside of disease, which may affect autonomic function. It has been shown that with age autonomic 
function values tend to worsen $27,64,65,114$ and that women tend to have worse values

of autonomic function than men 65,114 . In the present study, the three groups (Control, NonDM, and DM) were essentially equivalent on both age and gender. No studies were found which documented any affect of race on autonomic function. Thus, it is unknown if, or how, the $89 \%$ Caucasian composition of the control group affected the values obtained.

It was quite interesting that all groups ranked the symptoms and impact scores in the same order. Impotence appeared to be the major symptom and problem for men with hypoglycemia unawareness ranking a close second for the patients with diabetes and first for women with diabetes. For symptom categories responded to by the group as a whole, gastrointestinal problems are of major concern, with women without diabetes ranking them first.

\section{Reliability}

Reliability was found to be moderate to high using pretest/posttest correlation scores for symptom categories. The fluctuation seen in the reliability coefficients could be due to the small sample size for both the controls and patients ${ }^{117}$. Burns and Grove $^{116}$ suggest that a well-developed measurement instrument should have a reliability of at least 0.8 , whereas a newly developed instrument should have a reliability of 0.7. Most of the ASC categories have a reliability of at least 0.7 , and meet the criteria for reliability. 


\section{Implications and Conclusions}

The ASC is a potentially useful research instrument for monitoring symptom changes brought about by interventions designed to improve autonomic function, such as transplantation and exercise. It is also important to note that a question to quantify how important or bothersome a category of symptoms is, if present, included in each category. Only one impact question is asked in each category (versus an impact question for each symptom question) in an assumption that it is more important to obtain a global quantification of the category impact and in an effort to simplify the questionnaire. This question is an initial effort to address quality of life of patients as it relates to symptoms of dysautonomia.

The ASC was designed to ascertain ESRD patients' perceptions of symptoms, therefore, it is intended to be self-administered, and is subjective in nature. Presently, it is being used in a longitudinal research protocol as an instrument to obtain subjective data in conjunction with two objective tests of autonomic function. The ASC was not designed to provide objective clinical signs of dysautonomia. The ASC is easy to administer, has a Flesch-Kincaid reading grade level of 3.3, and has only four to six questions addressing each of the five symptom categories. This design was thought to improve patient compliance in completing and returning the instrument.

To increase the variability in scores, further refinements to the ASC are needed. Potential revisions include replacing the "never" and "always" Likert anchors with more descriptive anchors, including in the directions instructions to use the past one to two months as a time-frame when deciding how to answer the questions, and clarifying the wording of some of the questions. Further studies to confirm reliability using a larger 
sample size are needed; however, r-values, even with a small sample size, were moderate to high.

One of the advantages for use of this instrument are the individual category symptom scores and impact scores. Category scores consequently can be correlated with specific physiologic measurements of dysautonomia. For example, the Orthostatic Hypotension symptom category can be correlated with objective laboratory evoked tests, which evaluate systolic blood pressure change when the patient moves from a lying to a standing position. Thus, if a patient is experiencing symptoms of dysautonomia in only a few categories, the categories can be identified, quantified, and compared to objective test results. Correlation of individual category scores or the ASC index score with objective test measures could help identify the threshold where autonomic function test values herald the presence of symptoms. If specific thresholds are identified, interventions could feasibly be designed to prevent progression to symptomatology.

The consistent finding of low symptom scores suggests a relatively asymptomatic state, even for ESRD groups with severe dysautonomia as measured by physiologic testing, supporting the premise that symptoms of dysautonomia occur late in the disease process 61 . For this reason, the ASC is not designed as a screening tool for dysautonomia, but rather as a research instrument to be used in combination with other measures of autonomic function. Within a research environment, the ASC could be used to discover when, in the progression of disease, do symptoms occur, and in a clinical setting to monitor the progression of disease and effects of therapy. To develop a screening tool for early dysautonomia, early symptoms of dysautonomia, if they can be identified, would have to be established. 
In conclusion, the ASC was found a valid and reliable research instrument for assessing symptoms of dysautonomia in the ESRD transplant population. If clinicians could begin treating the dysautonomia prior to symptoms, the onset of symptoms with subsequent deterioration in quality of life could possibly be delayed. In addition, the ASC's clinical utility for monitoring changes in autonomic symptomatology resulting from transplantation was established. Thus, the ASC can be incorporated into longitudinal research protocols. The next step in a program of research would be to correlate physiological measures of dysautonomia to symptomatology in hopes of identifying a threshold, which identifies the beginning of symptomatology. 


\section{Chapter 3. Establishment of Referent Values for Evoked Autonomic Function, 24-Hr Heart Rate Variability, and Autonomic Symptomatology}

In 1988, a consensus statement written by the American Diabetes Association and the American Academy of Neurology was published 118 . The organizations recommended that when performing evoked autonomic function tests, a battery of tests should be used and each laboratory should standardize their own cardiovascular measures using the population they were studying. Each laboratory, in addition, should establish it's own normal referent values from an appropriate normative control population. No recommendations were made for testing autonomic function with 24-hr HRV analysis. Four years later, in 1992, the two groups again met to review the current state of the art of standardized autonomic function testing. The second consensus group now agreed that evoked measures were sufficiently standardized for longitudinal assessment of cardiovascular autonomic function of patients with diabetes. The recommended evoked battery of tests included the change in heart rate with deep breathing and the Valsalva maneuver 21 . In addition, physiologic factors, such as respiratory rate and age, and confounding variables, such as smoking and dehydration, which are thought to affect tests results, were identified. However, evaluating autonomic function using ambulatory monitoring was only briefly mentioned with no recommendation for testing or information on sensitivity and specificity. 
Recommendations for standardized nomenclature and methods of measurement for heart rate variability 53 were made by the Task Force of the European Society of Cardiology and the North American Society of Pacing and Electrophysiology in 1996. These recommendations included which time- and frequency-domain measures to report, guidelines for standardization of ECG recording length and analysis, and technical requirements for commercial equipment. It is yet to be seen how these recommendations will be utilized in clinical research.

Autonomic function testing is an evolving field. This is reflected in the fact that although recommendations for standardization of autonomic function testing research methodologies have been made $4,21,53$, a standard methodology and reporting system is not used in all laboratories. Thus, it is difficult to compare and contrast research findings among various studies due to different research protocols, analysis techniques, and reporting formats. This study was designed to establish normal, borderline, and abnormal values for measures of autonomic function and to determine if these values can distinguish healthy individuals from ESRD patients. In addition, the extent to which age, gender and presence or absence of ESRD and diabetes mellitus influence measures of cardiovascular autonomic function was explored. To this purpose, the following research questions were investigated.

1. What responses to evoked cardiovascular function testing, 24-hr HRV monitoring, and autonomic symptomatology are characteristic of

1a) healthy adult individuals?

1b) adult ESRD pretransplant patients? 
2. What is the relationship between age and measures of evoked cardiovascular function, 24-hr HRV monitoring, and autonomic symptomatology for

2a) healthy adult individuals?

b) adult ESRD pretransplant patients?

3. What is the difference between measures of evoked cardiovascular function, 24-hr HRV monitoring, and autonomic symptomatology for

3a) healthy adult men and women individuals?

3b) adult men and women ESRD pretransplant patients?

4. What are the relationships among measures of autonomic function in 4a) healthy adult individuals?

4b) adult ESRD pretransplant patients?

5. What are normal, borderline, and abnormal values for evoked autonomic tests, 24-hr HRV, and Autonomic Symptom Checklist measures?

\section{Introduction}

The heart rate response to the Valsalva maneuver and change in heart rate with deep breathing (HRDB) are widely used, simple tests which quantify cardiovascular autonomic function $3,4,15$. The Valsalva ratio (VR) is determined by calculating changes in heart rate during and following a Valsalva maneuver. An early study 19 examined the VR of 42 patients with diabetes mellitus and found this measure an objective assessment of the state of the autonomic nervous system in diabetic patients. At that time, Ewing defined VR values $\leq 1.10$ as abnormal, 1.11 to 1.20 as borderline, 
and values $\geq 1.21$ as normal $^{19}$. Mayo Clinic $^{73}$ has subsequently established control values that are age-related (see Table 14).

In the 1970s, HRDB was reported to be a simple, accurate 119,120 and reproducible ${ }^{119}$ method to test cardiac vagal neuropathy in diabetic patients. Mackay, Page, Cambridge, and Watkins 63 and Dyrberg, Benn, Christiansen, Hilsted, and Nerup 22 defined HRDB as abnormal when the change in beats between inspiration and expiration was less than 9, which was the lower limit of normal for the control group 22 . For research purposes, Ewing, Martyn, Young, and Clarke 3 established the commonly accepted values for HRDB to be $\leq 10$ as abnormal, 11 to 14 as borderline, and $\geq 15$ as normal. Different values have been established by Mayo Clinic 73 (see Table 14). In the 1980s, researchers began describing power spectral analysis of $\mathrm{HRV}$ as a quantitative method to evaluate autonomic regulation of the cardiovascular system ${ }^{9}$ and began examining HRV in both time- and frequency-domains ${ }^{121}$. Once HRV was recognized as a strong and independent predictor of mortality after an acute myocardial infarction 37,122 , its clinical importance was appreciated. Normal values for $24-\mathrm{hr}$ time and frequency measures were established by Bigger et al. ${ }^{2}$ by recruiting 274 healthy middle-aged individuals (range $40-60$ years old, mean $57 \pm 8.2$ years, $74 \%$ men) (see Table 15). These same values were accepted as normal by the Task Force of the European Society of Cardiology and the North American Society of Pacing and Electrophysiology 53 . 
Table 14. Varying Normal, Borderline, and Abnormal Values for Evoked Measures

Normal Borderline Abnormal

\section{Change in HR with Deep Breathing}

Values established by Ewing et al. 3

$\geq 15$

$11-14$

$\leq 10$

Control Values established by Mayo Clinic $^{73}$

10-40 yr. olds

$\geq 18$

41-50 yr. olds

$\geq 16$

51-60 yr. olds

$\geq 12$

61 and over

$\geq 8$

Values established by MacKay et al. ${ }^{63}$ and

Dyrberg et al. 22

Values established by Naverro et al. $41,123 \geq 15$

\section{Valsalva Ratio}

Values established by Ewing et al. 19

$\geq 1.21$

$1.11-1.20$

$\leq 1.10$

Control Values established at Mayo Clinic $^{73}$

10-40 yr. olds

$\geq 1.50$

41-50 yr. olds

$\geq 1.45$

51-60 yr. olds

$\geq 1.45$

61 and over

$\geq 1.35$

Values established by Navarro et al. $41,123 \geq 1.43$ 
Table 15. Characteristic Values for Healthy Middle-aged Persons (Selected 24-hr Time- and Frequency-Domain Measures) ${ }^{2}$

\begin{tabular}{lr}
\hline Measure & $\begin{array}{r}\text { Healthy Subjects } \\
(\text { mean } \pm \text { SD) }\end{array}$ \\
\hline SDNN (ms) & $141 \pm 39$ \\
SDANN (ms) & $127 \pm 35$ \\
RMSSD (ms) & $27 \pm 12$ \\
PNN50 & $9 \pm 7$ \\
Total power ${ }^{\mathrm{a}}(<0.4 \mathrm{~Hz})$ & $9.83 \pm 0.54$ \\
LF $^{\mathrm{a}}(0.04-0.15 \mathrm{~Hz})$ & $6.45 \pm 0.68$ \\
$\mathrm{HF}^{\mathrm{a}}(0.15-0.40 \mathrm{~Hz})$ & $5.05 \pm 0.83$ \\
\hline $\begin{array}{l}\text { Note. } \\
\text { time- and frequency-domain variables. }\end{array}$
\end{tabular}




\section{Cardiovascular Autonomic Function Measures}

It is well documented that autonomic function is attenuated in patients with ESRD $^{12,93}$ and in those with diabetes mellitus ${ }^{3}$. Valsalva ratio and HRDB, both of which are considered tests of primarily parasympathetic function 21 , have been commonly used to evaluate autonomic function in both patient populations. Only two studies 29, 93 were found which investigated cardiovascular autonomic function in dialysis patients. Chu, Tsai, Lee, and Yen ${ }^{29}$ examined 46 hemodialysis patients, 8 continuous ambulatory peritoneal dialysis patients, and 107 healthy adults. Results of this study found VR to be significantly less for dialysis patients as compared to the healthy controls, whereas, the HRDB did not differ significantly. In contrast, Hathaway,

Cashion, Milstead, Winsett, Cowan, Wicks, and Gaber93 found that both peritoneal $(n=61)$ and hemodialysis $(n=168)$ subgroups had diminished HRDB, but a normal VR. Clearly, further study is needed to document the characteristics and effect of dialysis on cardiovascular dysautonomia.

Autonomic function has been reported diminished in ESRD patients awaiting kidney or pancreas-kidney transplantation 93 . Ewing et al.'s values for normal HRDB and VR were used to interpret the findings $3,18,19$. When cardiovascular autonomic function was evaluated by diabetic status, nondiabetic ESRD $(n=184)$ had a normal HRDB and VR. In contrast, HRDB was abnormal in ESRD patients with either type 1 $(n=60)$ or type 2 diabetes $(n=34)$, and VR was borderline for both diabetes subgroups. In another study 99 , it was reported that following pancreas-kidney $(n=23)$ or kidney alone $(n=16)$ transplantation, kidney recipients experienced nonsignificant improvement in 
HRDB and VR. In contrast, the pancreas-kidney group demonstrated significant improvement in VR and insignificant improvement in HRDB. Thus, a measure thought to be reflective of parasympathetic function (HRDB) showed less change than VR, a measure increasingly thought to reflect some adrenergic function in addition to parasympathetic function 32 .

In a 1990 study of ESRD patients with type 1 diabetes mellitus who were awaiting pancreas transplantation, abnormal VR and HRDB were found in $75 \%$ of 232 patients 123 . In a subsequent larger study, abnormal values were found in $65 \%$ of 545 patients 41 . In both studies, normal values of HRDB and VR were established to be $\geq$ 15.0 and $\geq 1.43$, respectively $41,112,123$.

Evoked tests, HRDB and VR, have documented dysautonomia in pre and posttransplant ESRD patients. However, referent values tend to vary by laboratory and study population with no consensus on which test is the most sensitive.

\section{4-hr HRV Measures}

Twenty-four hour HRV values were diminished in studies of patients with diabetes $^{48,49}$, patients with uremia 87,93 , and patients with uremia and diabetes $87,93,109$. Bigger, Fleiss, Rolnitzky, Steinman, and Schneider 77 found 24-hr frequency-domain measures of 68 myocardial infarction patients obtained immediately, and at 12 months post-infarction, to be one half to two thirds the values found in a sample of 95 healthy individuals. These results were confirmed in a second study with 962 postinfarction patients ${ }^{2}$. 
Power spectral analysis of HRV demonstrated poorer values in a diabetic sample $(\mathrm{n}=23)$ when compared to healthy controls $(\mathrm{n}=22)$, and were especially pronounced in diabetic subjects below 65 years of age ${ }^{50}$. Within 3 weeks of renal transplantation, Kirvela, Salmela, Toivonen, Koivusalo, and Lindgren 86 obtained 24-hr HRV recordings on 12 diabetic and 11 non-diabetic renal transplant patients, in addition to 12 control patients. HRV was analyzed in time- and frequency-domains and showed poorer 24-hr HRV in ESRD patients with diabetes than without diabetes immediately posttransplant. Short-term ECG recordings have also been used to assess sympathovagal balance. In a study of 21 nondiabetic hemodialysis ESRD patients before and 8 to 12 months after kidney transplantation, Strano, De Casstro, Urani, Ferrucci, Marcheselli, Poli, Berloco, Calcagnini, Cerutti, and Cortesini 88 found significant improvement in low and high frequencies.

Although in healthy adults time-domain measures do not discriminate components from different physiologic processes, time-domain measures are strongly correlated with the frequency-domain measures, which have been shown to discriminate ${ }^{124}$. Thus, in research studies, correlation of time- and frequency-domain measures may be helpful when interpreting findings. Bigger et al. ${ }^{2}$ found that total power is strongly correlated with SDNN and SDANN. In addition, three commonly used measures of parasympathetic function, RMSSD, PNN50, and HF, are so highly correlated 46 that Bigger suggests these measures can be used interchangeably 82 . It is unknown if 24-hr measures continue to be highly correlated once the patient becomes chronically ill. 
Twenty-four hour HRV with power spectral analysis has confirmed poorer HRV exists for patients with chronic disease ranging from ESRD to coronary heart disease. This is an emerging technology and referent values tend to vary by laboratory and study population. Referent values and values characteristic of the pre and post transplant ESRD population need to be determined.

\section{Physiologic Factors}

In studies examining healthy individuals, the attenuation of both HRDB and VR with increasing age, independent of gender, appears to be a consistent finding 27,31 . However, findings were inconsistent on the ability to diagnose dysautonomia in subjects over the age of $65^{27}$ using HRDB or VR, with one study 31 finding that the diagnosis of dysautonomia was possible in subjects over 80 years of age. Gender was shown to have an effect on autonomic function, in some studies using evoked measures with women, having lower values 31 , while not in another study 27 . Findings, from studies using 24 -hr HRV measures to evaluate autonomic function, also indicate a decrease in autonomic function with increasing age in healthy individuals 2,65 . Women have, in addition, been found to have lower values in most measures 65 .

The influences of gender and age on autonomic function as measured by 24-hr HRV 65 appeared blunted in a group of 95 surviving sudden cardiac arrest patients with only the frequency measures decreasing with age. Bigger et al. ${ }^{2}$ recruited 274 healthy middle-aged individuals ( $57 \pm 8.2$ years old, $74 \%$ men) to establish normal values for 24 hr time- and frequency-domain measures. Findings showed a significant decrease in low and high frequency measures with increasing age and small, inconsistent differences 
between men and women. No reports were found on the influence of age and gender on autonomic function post-transplantation.

Although normal referent values have been suggested for evoked measures of autonomic function 3,19 , normal and abnormal values for 24-hr HRV measures are just beginning to be determined 2,53 and may vary depending on methodology and analysis techniques. Thus, researchers 29,112 frequently test healthy control groups for comparisons to a sample of their population under study. This is in accordance with recommendations by both the American Diabetes Association and the American Academy of Neurology 4 . Using this model, the purpose of this investigation was to establish values of evoked measures and 24-hr HRV measures, in addition to symptomatology, which were characteristic of the ESRD pre-transplant population and a comparison healthy control group. From the values characteristic of healthy individuals, normal borderline and abnormal values were determined.

\section{Methodology}

\section{Sample}

A convenience sample of ESRD patients pre-kidney or kidney-pancreas transplant $(n=363)$ were evaluated for dysautonomia. In addition, a group of healthy persons $(n=158)$ was recruited from faculty, family and friends to determine normal values for autonomic function in our Physiologic Function Lab and to compare healthy values to values from ESRD patients. Patients scheduled for pretransplant evaluation between February 1996 through February 1998 were asked to participate. All data obtained were a part of the patient's normally scheduled evaluation. All pretransplant patients over 18 
years of age were included in the study unless they were physically unable to participate in data collection or if they were found to have other neurologic disorders which could affect autonomic function values. Each patient with diabetes mellitus had blood glucose levels checked prior to autonomic function testing. If the blood glucose was $200 \mathrm{mg} / \mathrm{dl}$ or greater, the testing was cancelled and rescheduled. Demographic data included information on current medications, alcohol and tobacco use. Healthy control data were collected between November 1995 through February 1998. Subjects were considered healthy if they reported no neurologic dysfunction or coronary heart disease. This study was approved by the Institutional Review Board of the University of Tennessee, Memphis. Each participant signed an informed consent. Not all participants completed all three components of the evaluation; evoked tests, 24-hr HRV tests, and the Autonomic Symptom Checklist.

\section{Procedure}

Study participants were recruited and informed of the study. They were then scheduled for an appointment in the Physiologic Function Lab where the testing was again explained, a consent form (see Appendix B) was signed, demographic data obtained, evoked cardiovascular tests done, ASC completed, and an ambulatory Holter monitor applied. Participants were asked to return the Holter monitor within 36 to 48 hours. Following data collection, analysis was completed and reviewed weekly.

\section{Evoked Cardiovascular Tests}

Evoked measures, change in heart rate with deep breathing (HRDB) and the Valsalva ratio (VR) were obtained by tests performed in a temperature-controlled 
laboratory dedicated to performing autonomic function tests. Environmental stimuli such as noise and light were minimized. A Power Macintosh 9500 computer system and AcqKnowledge ${ }^{\circledR}$ III BIOPAC software were used for data acquisition and analysis of standard electrocardiogram recordings and blood pressure measurements obtained from a Dinamap ${ }^{\mathrm{TM}}$ XL (CRITIKON). The protocols (see Appendix A) used to perform the evoked tests were modified from the Mayo Clinic's protocols ${ }^{73}$, and a brief overview follows.

The HRDB value was obtained while the patient was relaxed in a recumbent position breathing deeply and regularly for one minute at a rate of 6 breaths per minute ( 5 second inhale/5second exhale) which follows a two minute period of regular breathing. The difference between the maximum and minimum heart rates was calculated (see Appendix A). The second test, the VR, was derived by dividing the maximum heart rate during a seated patient's forced expiration of $40-\mathrm{mm} \mathrm{Hg}$ for 15 seconds by the minimum heart rate within 15 seconds after the maneuver (see Appendix A). Reliability and validity have been established for HRDB and VR $5,19,23,63$.

\section{Ambulatory 24-hr Holter Recordings}

Data collection and processing of 24-hr HRV measures followed recommendations 125 , and are briefly summarized here. Analysis of 24-hr ambulatory Holter monitor tapes was completed by Marquette Electronics Laser SXP® Ambulatory ECG Analysis and Editing Systems with version 5.8 software program and Series 8500 Holter recording system (see Appendix A). Each QRS complex was digitized, identified and labeled. The analyzed data file was then scanned and manually edited to locate and 
correct any errors in QRS labeling that would adversely affect measurement of heart rate. Tapes were required to have $\geq 20$ hours of analyzable data and generally had $\geq 23$ hours of analyzable data. Using these data files, 24-hr HRV with power spectral analysis was calculated.

The time-domain HRV calculations are placed in 2 general categories. The first category is derived directly from the R-R intervals and includes means and standard deviations of the interval. Measures in this category of the time-domain include the SDNN and the SDANN. The SDNN is the standard deviation of all R-R intervals during the 24-hours and has been found to be associated with sudden cardiac death ${ }^{37}$. The SDANN is the standard deviation of the means of R-R intervals found in successive five minute blocks over 24-hours and is considered the best measure of overall autonomic balance and represents circadian rhythmicity of autonomic function 75 , in addition to estimating long-term components of HRV ${ }^{125}$. The second category of time-domain variables is based on the differences between adjacent $R-R$ intervals and included the RMSSD. The RMSSD is the square root of the mean of the sum of squares of differences between adjacent R-R intervals. The RMSSD is virtually independent of circadian rhythms and reflects alterations in autonomic function that are primarily vagally mediated 57,125 (see Table 1).

The power spectrum is computed over the 24-hr recording interval using fast Fourier transform for 3 frequency bands; total $(0.01-1.00 \mathrm{~Hz})$, low $(0.04-0.15 \mathrm{~Hz})$ and high $(0.15-0.40 \mathrm{~Hz})$ frequency power. Low frequency waveforms reflect modulation of sympathetic and parasympathetic activity by baroreflex mechanisms while high 
frequency waveforms, also known as the respiratory frequency, represent modulation of parasympathetic activity by respiration 9,10 .

\section{Autonomic Symptom Checklist}

The aims of the ASC were to recognize the perceived presence, distribution and severity of autonomic symptoms. Initially, the instrument was compiled using information from several expert sources $59,61,115$. Subsequently, a focus group met over a five-week period for four, two hour sessions to refine the questions and simplify the reading level of the instrument. The focus group was comprised of people with experience as transplant coordinators, diabetic educators, and researchers in addition, one person had type 2 diabetes mellitus. Following focus group discussions, the ASC was pilot tested on four ESRD patients and further revisions were made. Revisions included clarifying terminology and adding a time-frame to the directions.

The revised ASC was developed as a self-administered checklist that assessed dysautonomia symptoms in five categories with 32-items each with a 7-point Likert scale $^{116}$ (see Appendix C). A self-administered format was chosen because, typically, the individual's perception of these symptoms is what alerts the provider to the possibility of dysautonomia. Three categories, Orthostatic Hypotension, Sudomotor Abnormalities, and Gastrointestinal Problems, are to be completed by all respondents, while two categories are also completed by subgroups: the Impotence category is to be completed by men; and the Hypoglycemia Unaware is to be completed by individuals with diabetes mellitus. 
Each of the five categories consists of four to six questions, each answered using a seven-point Likert scale with 0 being "never" and 6 being "always". The individual is asked to circle the number that indicates the severity of that symptom. The last question in each category is classified as an "impact" question and used to obtain a score reflecting patients' perceptions of how problematic that category of symptoms is for them. This question was included because the presence of a symptom and the degree to which a symptom creates problems are not always the same. In addition, information is obtained concerning each patient's current medications, alcohol and caffeine intake, and tobacco use. These products could affect function of the autonomic nervous system ${ }^{21}$, but it is unknown as to what extent.

The Orthostatic Hypotension category consists of five questions (30 points possible) focused on symptoms of hypotension upon standing. These symptoms include rapid heartbeat, blurred vision, an upset stomach, dizziness, and clammy skin. The Sudomotor Abnormalities category consists of three questions (18 points possible) focused on an increase or decrease in sweating. The Gastrointestinal Problems category consists of five questions (30 points possible) focusing on bowel and stomach disorders. The Impotence category consists of four questions ( 24 points possible) focused on the ability to have and maintain an erection. The Hypoglycemia Unaware category consists of four questions (24 points possible) focused on subjects' ability to recognize symptoms associated with a low blood sugar. Each category of symptom questions is followed by an impact question "how much of a problem are these symptoms for you?" (6 points possible). 
Patient responses are entered into the database as scored by the individual, except for questions $4 \mathrm{a}, 4 \mathrm{~b}$, and $4 \mathrm{c}$ in the Impotence category and question $5 \mathrm{a}$ in the Hypoglycemia Unawareness category, which are written in reverse fashion and, therefore, scored in reverse fashion. Category and index calculations are performed based on these values with higher scores indicating greater symptoms. See Appendix D for definitions and a detailed description of the calculations.

For each patient, two scores per category are reported: symptom score and impact score. The category symptom score was calculated by obtaining a raw score for each category then dividing the raw score by the number of questions in that category. Each category symptom score has a range from $0-6$. The category impact score is obtained directly from the one impact question and also ranged from $0-6$. Each category symptom score and impact score can be compared across groups.

Two other scores reported are total symptom score and total impact score. The total symptom score (6 points possible) are the sum of category symptom scores divided by the number of categories reported (e.g., 5 for DM women, 6 for DM men). The total impact score (6 points possible) is calculated by dividing the total number of points scored on the category impact questions by the number of impact questions possible for that group. Both the total symptom score and the total impact score can be compared across groups.

The ASC index score (36 points possible) is a single measure designed to reflect the patient's perception of experienced dysautonomia symptoms and the impact of those symptoms on the patient. It is calculated by multiplying the total symptom score and the total impact score and can be compared across groups. 


\section{Data Analysis}

For analysis, only one baseline pretransplant evoked test, 24-hr HRV test, and ASC test was used for each subject in each group. In addition, subjects with type 2 diabetes mellitus were excluded from analysis; thus, the ESRD with DM group was composed of patients with only type 1 diabetes mellitus. This prevented any confounding effects that might occur due to differing physiologic mechanisms causing the dysautonomia in type 1 and type 2 diabetes mellitus patients. Expected values for each study measure were determined for the healthy control group with the aim of using these data to establish normal, abnormal, and borderline values for each of the study measures. Using the means and SDs for each evoked and 24-hr HRV measure, values were determined for each study measure at 2 SDs below the mean, the lower $95 \%$ confidence interval, and the 2.3 percentile. These values were defined as abnormal and then scatterplots were used to visually analyze each measure for cutpoints to determine if they logically identified a group that was abnormal. In addition, these cutpoint values were applied to each study group to determine what percent of each group was classified as normal and abnormal. Methods for determining referent values were adapted from several studies $2,19,35,63,109$. Chi square tests were used to determine equivalence of groups for categorical data. One way ANOVA with preplanned multiple comparisons using Least Square Means was used to test for differences among study groups. Correlational analysis was used to evaluate the relationship between age, gender, and all measures. 


\section{Results}

Results will first be reported for autonomic function and symptomatology characteristic of healthy controls $(n=158)$ and 2 samples of ESRD patients (ESRD with DM, n=91; ESRD NonDM, n=272) followed by the relationships between age, gender and autonomic function. In addition, referent values for evoked measures, 24-hr HRV measures, and the Autonomic Symptom Checklist will be established.

\section{Autonomic Function of Healthy Controls and Two ESRD Samples}

Although the ages of the three study groups were similar (see Table 16) with healthy controls being slightly younger than ESRD patients with diabetes mellitus (ESRD DM) and ESRD patients without diabetes mellitus (ESRD NonDM) groups $(36.2,39.5$, 41.9; respectively;), there was a significant difference in age $(\mathrm{p} \leq 0.001)$ between the healthy and ESRD NonDM groups. In addition, there was a significant difference in gender $(\mathrm{p} \leq 0.001)$ with healthy controls being primarily women $(75 \%)$ as compared to both ESRD DM (47\%) and ESRD NonDM (37\%) groups. However, both healthy controls and ESRD DM groups were 77 \% Caucasian as compared to the ESRD NonDM group which was significantly different $(\mathrm{p} \leq 0.001)$ with $56 \%$ African-American. There was no difference among the three groups when compared by exercise, with $29-36 \%$ of each group reporting that they exercise. The ESRD NonDM group had significantly $(\mathrm{p} \leq 0.0001)$ more months of dialysis $(27.7 \pm 34.4)$ when compared to the ESRD DM group $(8.0 \pm 12.5)$ 
Table 17 shows data generated in response to the research question, "what are the evoked, 24-hr HRV, and Autonomic Symptom Checklist values characteristic of ESRD pretransplant adult patients and healthy adult individuals?" The average RR interval (mean NN) was found to be longer for the healthy group $(746 \pm 77)$ compared to the ESRD NonDM group $(724 \pm 103)$ and significantly longer than the ESRD DM group $(705 \pm 94 ; \mathrm{p} \leq 0.01)$. Thus, the healthy group had a slower heart rate than the other 2 groups. Excluding the mean NN, all other measures of evoked and 24-hr HRV were 
Table 16. Characteristics of Healthy Controls, ESRD Pretransplant Patients, ESRD Patients with Diabetes Mellitus (DM), and ESRD Patients Without Diabetes Mellitus (NonDM)

\begin{tabular}{|c|c|c|c|c|c|}
\hline & & $\begin{array}{c}\text { ESRD } \\
\text { with DM } \\
(n=91)\end{array}$ & $\begin{array}{c}\text { ESRD } \\
\text { NonDM } \\
(\mathrm{n}=272)\end{array}$ & $\begin{array}{c}\text { Total ESRD } \\
\text { Sample } \\
(\mathbf{n}=\mathbf{3 6 3})\end{array}$ & $\begin{array}{l}\text { Healthy } \\
\text { Controls } \\
(n=158)\end{array}$ \\
\hline \multicolumn{6}{|l|}{ Characteristic } \\
\hline Age (yrs.) & $\begin{array}{r}\text { mean } \pm \mathrm{SD} \\
\text { range }\end{array}$ & $\begin{array}{r}39.5 \pm 8.1 \\
25-67\end{array}$ & $\begin{array}{r}41.9 \pm 12.1^{*} \\
18-69\end{array}$ & $\begin{array}{r}41.3 \pm 11.3 \\
18-69\end{array}$ & $\begin{array}{r}36.2 \pm 11.2^{*} \\
18-65\end{array}$ \\
\hline \multicolumn{6}{|l|}{ Gender } \\
\hline Men & $\mathrm{n}(\%)$ & $48(53)^{*}$ & $172(63)^{*}$ & $220(60)$ & $40(25)^{*}$ \\
\hline Women & $\mathrm{n}(\%)$ & $43(47)^{*}$ & $100(37)^{*}$ & $143(40)$ & $118(75)^{*}$ \\
\hline \multicolumn{6}{|l|}{ Race } \\
\hline African-Amer. & $\mathrm{n}(\%)$ & $21(23)^{*}$ & $182(67)$ & $203(56)$ & $29(19)^{*}$ \\
\hline Caucasian & $\mathrm{n}(\%)$ & $70(77)^{*}$ & $89(33)$ & $159(44)$ & $121(77)^{*}$ \\
\hline Other & $\mathrm{n}(\%)$ & 0 & $1(0.4)$ & $1(0.2)$ & $7(4)$ \\
\hline \multicolumn{6}{|l|}{ Exercise } \\
\hline Yes & $\mathrm{n}(\%)$ & $27(32)$ & $99(29)$ & $126(37)$ & $43(36)$ \\
\hline No & $\mathrm{n}(\%)$ & $58(68)$ & $153(61)$ & $211(63)$ & $78(64)$ \\
\hline Dialysis Type & & & & & na \\
\hline HomeHemo & $\mathrm{n}(\%)$ & $1(1)$ & $4(2)$ & $5(2)$ & \\
\hline Incenter Hemo & $\mathrm{n}(\%)$ & $24(32)$ & $134(62)$ & $158(54)$ & \\
\hline Peritoneal & $\mathrm{n}(\%)$ & $17(23)$ & $55(26)$ & $72(25)$ & \\
\hline None & $\mathrm{n}(\%)$ & $33(44)$ & $22(10)$ & $55(19)$ & \\
\hline Months dialysis & mean $\pm \mathrm{SD}$ & $8.0 \pm 12.5^{*}$ & $27.7 \pm 34.4^{*}$ & $22.6 \pm 31.4$ & na \\
\hline Years of DM & mean $\pm \mathrm{SD}$ & $24.8 \pm 6.9$ & na & & na \\
\hline Unemployed & $\mathrm{n}(\%)$ & $52(58)$ & $150(55)$ & $88(56)$ & unknown \\
\hline Height (cm) & mean \pm SD & $168.0 \pm 9.3$ & $169.0 \pm 10.5$ & $168.8 \pm 10.2$ & unknown \\
\hline Weight (kg) & mean \pm SD & $73.3 \pm 14.1$ & $76.5 \pm 16.9$ & $75.7 \pm 16.2$ & unknown \\
\hline
\end{tabular}

Note. $* \mathrm{p} \leq 0.001$ between groups with like symbols. $\mathrm{ESRD}=$ end-stage renal disease, $\mathrm{DM}=$ diabetes mellitus, NonDM=without diabetes mellitus, Hemo=hemodialysis 
significantly ( $\mathrm{p} \leq 0.001)$, and, substantially, poorer in patients with ESRD compared to healthy individuals. In addition, almost all measures were significantly more compromised for ESRD patients with diabetes mellitus compared to ESRD patients without diabetes. A higher score on the ASC index reflects a heightened perception of dysautonomia symptoms and the impact those symptoms have on the individual. Similar to the objective measures, the ESRD patients reported significantly greater symptomatology $(\mathrm{p} \leq 0.001)$ than the healthy controls $(0.12 \pm 0.21)$, with ESRD patients with diabetes reporting greater symptomatology (4.79 \pm 5.2$)$ than the ESRD patients without diabetes $(2.09 \pm 3.3)$.

\section{Relationship Between Age and Autonomic Function of Healthy Controls and Two ESRD Samples}

For each of the 3 study groups, a correlational analysis was computed for the two evoked measures, the 3 frequency-domain measures, the 6 time-domain measures, the ASC index and age. Tables 18 shows the data generated in response to the research question, "Is there a relationship between age and the evoked cardiovascular test, 24-hr HRV test, and Autonomic Symptom Checklist values of adult ESRD patients and healthy individuals?" 
Table 17. Comparisons of Evoked, 24-hr Heart Rate Variability, and ASC Measures Among Healthy Controls $(n=158)$ and ESRD Pretransplant Patients $(\mathbf{n = 3 6 3 )}$ With (ESRD DM=91) and Without Diabetes (ESRD NonDM=272)

\begin{tabular}{|c|c|c|c|c|}
\hline & $\begin{array}{c}\text { ESRD with } \\
\text { DM }\end{array}$ & $\begin{array}{c}\text { ESRD } \\
\text { NonDM }\end{array}$ & $\begin{array}{c}\text { Total ESRD } \\
\text { Sample }\end{array}$ & Healthy Controls \\
\hline \multicolumn{5}{|c|}{ Evoked measures } \\
\hline HRDB & $11.2 \pm 9.9 \dagger$ & $18.0 \pm 9.9 \dagger$ & $16.2 \pm 10.3 *$ & $26.1 \pm 9.8 * \dagger$ \\
\hline VR & $1.22 \pm 0.38 \dagger$ & $1.38 \pm 0.40 \dagger$ & $1.34 \pm 0.40^{*}$ & $1.68 \pm 0.40^{*} \dagger$ \\
\hline \multicolumn{5}{|c|}{ 24-hr HRV measures } \\
\hline Total power ${ }^{\mathrm{a}}$ & $4.57 \pm 1.30 \dagger$ & $5.82 \pm 1.05 \dagger$ & $5.51 \pm 1.24 *$ & $7.25 \pm 0.63 * \dagger$ \\
\hline $\mathbf{L F}^{\mathbf{a}}$ & $3.10 \pm 1.70 \dagger$ & $4.7 \pm 1.27 \dagger$ & $4.31 \pm 1.54^{*}$ & $6.42 \pm 0.66^{* \dagger}$ \\
\hline $\mathbf{H F}^{\mathrm{a}}$ & $2.63 \pm 1.10 \dagger$ & $3.64 \pm 1.27 \dagger$ & $3.39 \pm 1.26^{*}$ & $5.20 \pm 0.91 * \dagger$ \\
\hline SDNN & $64 \pm 27 \dagger$ & $88 \pm 36 \dagger$ & $82 \pm 35^{*}$ & $134 \pm 33^{*} \dagger$ \\
\hline SDANN & $58 \pm 23 \dagger$ & $78 \pm 33 \dagger$ & $73 \pm 32^{*}$ & $120 \pm 32 * \dagger$ \\
\hline SD & $21 \pm 17 \dagger$ & $33 \pm 16 \dagger$ & $30 \pm 17 *$ & $58 \pm 15^{*} \dagger$ \\
\hline RMSSD & $13.2 \pm 12.6 \dagger$ & $19.0 \pm 12.0 \dagger$ & $17.5 \pm 12.4^{*}$ & $32.8 \pm 13.0^{*} \dagger$ \\
\hline PNN50 & $1.62 \pm 6.30 \dagger$ & $3.57 \pm 6.60 \ddagger$ & $3.09 \pm 6.54^{*}$ & $11.03 \pm 8.26^{*} \dagger \dagger$ \\
\hline Mean NN & $705 \pm 94 \#$ & $724 \pm 103$ & $719 \pm 100^{\wedge}$ & $746 \pm 7^{\wedge} \#$ \\
\hline ASC Index & $4.79 \pm 5.20 \dagger$ & $2.09 \pm 3.30 \dagger$ & $2.73 \pm 3.99 *$ & $0.12 \pm 0.21 * \dagger$ \\
\hline
\end{tabular}


As indicated in Table 18, there was an inverse relationship between age and most measures of heart rate variability for all three groups, demonstrating that autonomic function decreases as an individual ages. In addition, the relationship was generally much stronger for the healthy control group with $r$ values primarily ranging from $-0.30(p$ $\leq 0.2)$ to $-0.60(\mathrm{p} \leq 0.003)$ when compared to the ESRD DM group with $\mathrm{r}$ values primarily ranging from $-0.03(\mathrm{p} \leq 0.68)$ to -0.31 ( $\mathrm{p} \leq 0.05)$ or the ESRD NonDM group with $\mathrm{r}$ values primarily ranging from $-0.02(\mathrm{p} \leq 0.03)$ to $-0.43(\mathrm{p} \leq 0.00)$. In the healthy individuals, the frequency-domain measures showed the strongest inverse relationship $(-0.60 ; \mathrm{p} \leq 0.003)$

\section{Relationship Between Gender and Autonomic Function of Healthy Controls and Two ESRD Samples}

Table 19 shows data generated in response to the question, "is there a gender difference between measures of evoked cardiovascular function, 24-hr HRV monitoring, and autonomic symptomatology for healthy adult individuals and ESRD pretransplant adult patients?" To answer this question, women and men were compared separately in each of the three study groups. As Table 19 shows, men demonstrated significantly better values $(\mathrm{p} \leq 0.01)$ than women in the healthy group for frequency-domain values of total power and LF and for time-domain values of SDNN, SDANN, and SD. In addition, women had a faster heart rate $(\mathrm{Mean} \mathrm{NN}=733 \pm 71, \mathrm{p} \leq 0.001)$ as compared to the men (781 \pm 82$)$. Although the evoked measure VR tended to be higher for men when 

Table 18. Correlational Analysis Between Age and Evoked and 24-hr HRV Measures for Each of the Three Study Groups

\begin{tabular}{|c|c|c|c|c|c|c|c|c|c|c|c|}
\hline \multirow[b]{3}{*}{ Group } & \multicolumn{2}{|c|}{ Evoked Measures } & \multicolumn{3}{|c|}{ Frequency Measures } & \multicolumn{6}{|c|}{ Time Measures } \\
\hline & HRDB & VR & TotalPower & $\mathrm{LF}$ & $\mathrm{HF}$ & SDNN & SDANN & SD & RMSSD & PNN50 & MeanNN \\
\hline & & & & & & & & & & & \\
\hline $\begin{array}{l}\text { ESRD DM } \\
n=91\end{array}$ & -0.29 & -0.31 & -0.05 & -0.00 & -0.08 & 0.11 & 0.12 & -0.03 & -0.05 & -0.05 & 0.13 \\
\hline $\begin{array}{l}\text { ESRD NonDM } \\
\mathrm{n}=272\end{array}$ & -0.43 & -0.32 & -0.32 & -0.33 & -0.34 & -0.05 & -0.02 & -0.24 & -0.24 & -0.21 & 0.12 \\
\hline $\begin{array}{l}\text { Healthy Controls } \\
n=158\end{array}$ & -0.45 & -0.30 & -0.60 & -0.59 & -0.60 & -0.38 & -0.34 & -0.47 & -0.48 & -0.40 & -0.10 \\
\hline
\end{tabular}

$\stackrel{\infty}{\infty}$ 
Table 19. Comparisons Between Women and Men of Evoked, 24-hr Heart Rate Variability, and ASC Measures Among Healthy and ESRD Patients as a Group, With (ESRD DM) and Without (ESRD NonDM) Diabetes.

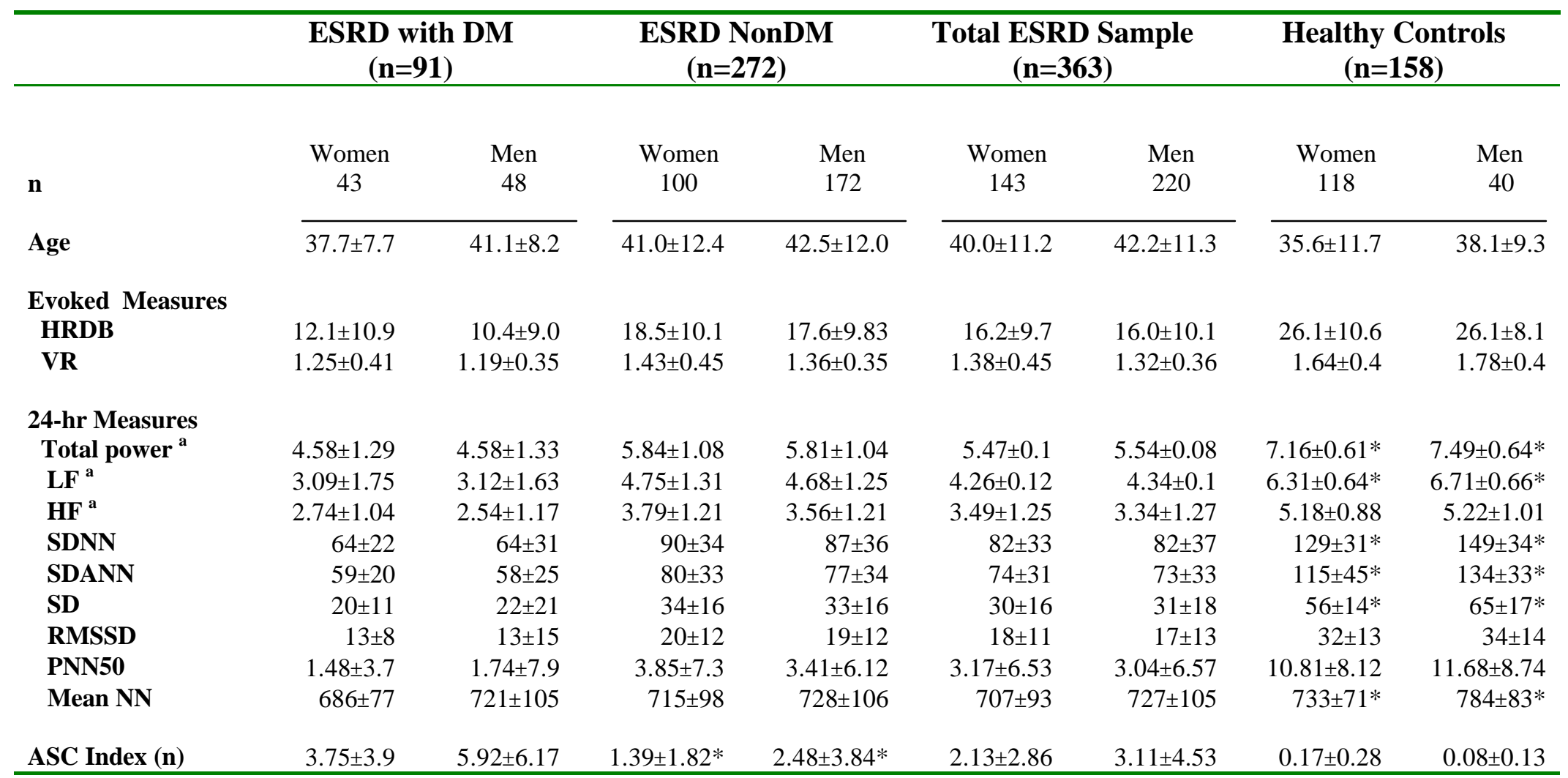

Note. $*$ and $\dagger \mathrm{p} \leq 0.05$ for groups having like symbols; ${ }^{a}=$ natural $\log (\mathrm{ms} 2)$. All values are given as mean $\pm \mathrm{SD}$. ESRD=end-stage renal disease, $\mathrm{DM}=\mathrm{diabetes}$ mellitus, NonDM=without diabetes mellitus. HRDB=heart rate change with deep breathing, VR=valsalva ratio. See Table 1 for $24-\mathrm{hr}$ HRV measure definitions 
compared to women, the difference failed to reach significance $(\mathrm{p} \leq 0.10)$, the HRDB was essentially the same for men and women. In contrast, the pattern of higher values for men compared to women was not seen in the 2 ESRD patient groups. Instead, men and women had similar values for most evoked and 24-hr HRV measures, all of which indicated compromised function. However, a significant difference $(\mathrm{p} \leq 0.05)$ was seen in ASC index between men $(2.48 \pm 3.84)$ and women (1.39 \pm 1.82$)$ in the ESRD NonDM group, with men reporting increased presence and impact of symptomatology.

\section{Relationships Among 24-hr HRV Measures of Autonomic Function}

For the healthy control group, many of the correlation coefficients among the time- and frequency-domain measures were strong, several exceeding the r-value of 0.90 (see Table 20). The pattern of strong r-values $(\mathrm{p} \leq 0.000)$ in the healthy group for total power and LF ( $r=0.99)$, total power and SD ( $r=0.97)$, LF and SD $(r=0.95), \mathrm{HF}$ and RMMSD ( $r=0.94)$, SDNN and SDANN ( $r=0.97)$ and RMSSD and PNN50 ( $r=0.98)$ was repeated in both ESRD groups. However, for most values the correlation coefficient, although still strong, decreased in the ESRD groups, with the weakest correlations seen in the ESRD DM group (see Tables 21 and 22).

\section{Referent Values for Evoked Measures, 24-hr HRV Measures, and Autonomic Symptomatology}

Data obtained from healthy individuals were first examined for normality and identification of outliers. Three individuals were identified whose results from 
Table 20. Correlational Analysis Among Evoked, Time- and Frequency-Domain Measures of 24-hr HRV in Healthy Controls $(\mathrm{n}=158)$

\begin{tabular}{|c|c|c|c|c|c|c|c|c|c|c|c|}
\hline & \multicolumn{2}{|c|}{ Evoked Measures } & \multicolumn{3}{|c|}{ Frequency Measures } & \multicolumn{6}{|c|}{ Time Measures } \\
\hline & HRDB & VR & Total power & er $\mathrm{LF}$ & $\mathrm{HF}$ & SDNN & SDANN & SD & RMSSD & PNN50 & MeanNN \\
\hline HRDB & 1.00 & & & & & & & & & & \\
\hline VR & 0.13 & 1.00 & & & & & & & & & \\
\hline Total power & 0.26 & -0.08 & 1.00 & & & & & & & & \\
\hline $\mathrm{LF}$ & 0.29 & -0.08 & 0.99 & 1.00 & & & & & & & \\
\hline $\mathrm{HF}$ & 0.40 & -0.17 & 0.88 & 0.86 & 1.00 & & & & & & \\
\hline SDNN & 0.15 & -0.11 & 0.82 & 0.80 & 0.66 & 1.00 & & & & & \\
\hline SDANN & 0.18 & -0.12 & 0.74 & 0.72 & 0.58 & 0.97 & 1.00 & & & & \\
\hline SD & 0.15 & -0.09 & 0.97 & 0.95 & 0.84 & 0.88 & 0.79 & 1.00 & & & \\
\hline RMSSD & 0.27 & -0.09 & 0.89 & 0.86 & 0.94 & 0.74 & 0.66 & 0.89 & 1.00 & & \\
\hline PNN50 & 0.20 & -0.10 & 0.85 & 0.82 & 0.88 & 0.72 & 0.64 & 0.87 & 0.98 & 1.00 & \\
\hline MeanNN & -0.07 & -0.20 & 0.71 & 0.67 & 0.60 & 0.77 & 0.67 & 0.81 & 0.68 & 0.69 & 1.00 \\
\hline
\end{tabular}

Note. See Table 1 for definitions of measures

ฉ̊ 
Table 21. Correlational Analysis Among Evoked Measures, Time- and Frequency-Domain Measures of 24-hr HRV in ESRD DM Patients $(\mathbf{n}=91)$

\begin{tabular}{|c|c|c|c|c|c|c|c|c|c|c|c|}
\hline & \multicolumn{2}{|c|}{ Evoked Measures } & \multicolumn{3}{|c|}{ Frequency Measures } & \multicolumn{6}{|c|}{ Time Measures } \\
\hline & HRDB & VR & Total power & $\mathrm{LF}$ & $\mathrm{HF}$ & SDNN & SDANN & SD & RMSSD & PNN50 & MeanNN \\
\hline HRDB & 1.00 & & & & & & & & & & \\
\hline VR & 0.70 & 1.00 & & & & & & & & & \\
\hline Total power & 0.58 & 0.47 & 1.00 & & & & & & & & \\
\hline $\mathrm{LF}$ & 0.61 & 0.48 & 0.97 & 1.00 & & & & & & & \\
\hline $\mathrm{HF}$ & 0.54 & 0.46 & 0.82 & 0.78 & 1.00 & & & & & & \\
\hline SDNN & 0.38 & 0.32 & 0.63 & 0.63 & 0.60 & 1.00 & & & & & \\
\hline SDANN & 0.35 & 0.29 & 0.53 & 0.54 & 0.52 & 0.99 & 1.00 & & & & \\
\hline $\mathrm{SD}$ & 0.51 & 0.45 & 0.93 & 0.92 & 0.84 & 0.68 & 0.57 & 1.00 & & & \\
\hline RMMSD & 0.32 & 0.30 & 0.61 & 0.56 & 0.83 & 0.43 & 0.34 & 0.70 & 1.00 & & \\
\hline PNN50 & 0.21 & 0.21 & 0.45 & 0.39 & 0.65 & 0.27 & 0.19 & 0.54 & 0.95 & 1.00 & \\
\hline MeanNN & -0.02 & -0.05 & 0.33 & 0.30 & 0.38 & 0.37 & 0.34 & 0.39 & 0.21 & 0.10 & 1.00 \\
\hline
\end{tabular}

Note. See Table 1 for definitions of measures.

우 
Table 22. Correlational Analysis Among Evoked Measures, Time- and Frequency-Domain Measures of 24-hr HRV in ESRD NonDM Patients $(n=272)$

\begin{tabular}{|c|c|c|c|c|c|c|c|c|c|c|c|}
\hline & \multicolumn{2}{|c|}{ Evoked Measures } & \multicolumn{3}{|c|}{ Frequency Measures } & \multicolumn{6}{|c|}{ Time Measures } \\
\hline & HRDB & VR & Total power & $\mathrm{LF}$ & $\mathrm{HF}$ & SDNN & SDANN & SD & RMSSD & PNN50 & MeanNN \\
\hline HRDB & 1.00 & & & & & & & & & & \\
\hline VR & 0.55 & 1.00 & & & & & & & & & \\
\hline Total power & 0.45 & 0.50 & 1.00 & & & & & & & & \\
\hline $\mathrm{LF}$ & 0.47 & 0.50 & 0.98 & 1.00 & & & & & & & \\
\hline $\mathrm{HF}$ & 0.42 & 0.47 & 0.88 & 0.82 & 1.00 & & & & & & \\
\hline SDNN & 0.28 & 0.41 & 0.73 & 0.70 & 0.65 & 1.00 & & & & & \\
\hline SDANN & 0.25 & 0.38 & 0.61 & 0.59 & 0.56 & 0.98 & 1.00 & & & & \\
\hline SD & 0.37 & 0.45 & 0.94 & 0.90 & 0.84 & 0.78 & 0.65 & 1.00 & & & \\
\hline RMMSD & 0.29 & 0.37 & 0.72 & 0.64 & 0.86 & 0.61 & 0.52 & 0.79 & 1.00 & & \\
\hline PNN50 & 0.26 & 0.34 & 0.60 & 0.52 & 0.73 & 0.56 & 0.48 & 0.72 & 0.95 & 1.00 & \\
\hline MeanNN & -0.12 & 0.00 & 0.51 & 0.44 & 0.51 & 0.51 & 0.44 & 0.59 & 0.54 & 0.46 & 1.00 \\
\hline
\end{tabular}

Note. See Table 1 for definitions of measures

$\stackrel{\infty}{\infty}$ 
possibility of unknown neurological problems. In addition, the healthy control data were examined for any individual test values that were greater than 3 SDs from the mean. Four healthy individuals were found who had one value each (either evoked or 24-hr HRV) that was more than 3 SDs above the mean. No healthy group values were found to be 3 SDs below the mean. Three healthy individuals were found to have ASC values more than 3 SDs above the mean. These values were deleted from the data and new means and SDs were determined for those study measures.

Abnormal values were determined for each study measure using 3 different methods; at 2 SDs below the mean 2,63 , the lower $95^{\%}$ confidence interval ${ }^{109}$, and the 2.3 quantile $^{35}$ (see Table 23). In addition, these cutpoint values were applied to each study group to determine what percent of each group was classified as normal and abnormal (see Table 23). Results showed that, for most measures, the 2.3 quantile best discriminated between normal and abnormal values in each of the groups. Thus, using the cutpoints established at the 2.3 quantile in the healthy group to classify the ESRD pretransplant groups, it was found in the ESRD DM group the frequency measures of total power, LF, and HF and the time-domain measure of SD best separated this group from the healthy group. For the ESRD NonDM group, the frequency measures of total power and LF and the time-domain measure of SD best separated this group from the healthy group.

After abnormal values were established using the 2.3 quantile cutpoint, borderline cutpoint values were established at the $5^{\text {th }}$ quantile (see Table 24). The values between abnormal ( 2.3 quantile) and normal $\left(\geq 5^{\text {th }}\right.$ quantile), were defined as borderline values. 
Table 23. Number and Percentage of Abnormal Test Responses in Healthy Controls, ESRD DM Patients, and ESRD NonDM Patients

\begin{tabular}{|c|c|c|c|c|}
\hline & & $\begin{array}{c}\text { ESRD with DM } \\
\text { n/ } /(\%)\end{array}$ & $\begin{array}{c}\text { ESRD NonDM } \\
\text { n }(\%)\end{array}$ & $\begin{array}{c}\text { Healthy Controls } \\
\text { n (\%) }\end{array}$ \\
\hline Measure & $\begin{array}{r}\text { Abnormal } \\
\text { Value }\end{array}$ & & & \\
\hline \multicolumn{5}{|l|}{ Evoked } \\
\hline -2SD & $\leq 6.0$ & $36 / 90(40)$ & 28/262 (11) & 0/98 (0) \\
\hline $95 \%$ CI & $\leq 7.0$ & $47 / 90(52)$ & 37/262 (14) & 0/98 (0) \\
\hline 2.3 quantile & $<10.0$ & $55 / 90(61)$ & 66/262 (25) & 2/98 (2) \\
\hline \multicolumn{5}{|l|}{ VR } \\
\hline$-2 S D$ & $\leq 0.88$ & $1 / 82(1)$ & $0 / 249(0)$ & $0 / 96(0)$ \\
\hline 95\% CI & $\overline{\leq} 0.90$ & $1 / 82(1)$ & 0/249 (0) & $0 / 96(0)$ \\
\hline 2.3 quantile & $\leq 1.06$ & $38 / 82(46)$ & $39 / 249(16)$ & $2 / 96(2)$ \\
\hline \multicolumn{5}{|l|}{ 24-hr HRV } \\
\hline \multicolumn{5}{|l|}{ Total power ${ }^{a}$} \\
\hline$-2 S D$ & $\leq 5.99$ & $70 / 79$ (89) & 139/243 (57) & $3 / 124(2)$ \\
\hline 95\% CI & $\leq 6.01$ & 70/79 (89) & 139/243 (57) & 4/124 (3) \\
\hline 2.3 quantile & $\leq 5.94$ & $69 / 79(87)$ & $136 / 243(56)$ & 2/124 (3) \\
\hline \multicolumn{5}{|l|}{$\mathbf{L F}^{\mathbf{a}}$} \\
\hline$-2 S D$ & $\leq 5.10$ & 69/79 (87) & 145/243 (60) & 4/124 (3) \\
\hline $95 \%$ CI & $\leq 5.13$ & 71/79 (90) & 149/243 (61) & 5/124 (4) \\
\hline 2.3 quantile & $\leq 4.96$ & $69 / 79(87)$ & 134/243 (55) & $2 / 124(2)$ \\
\hline \multicolumn{5}{|l|}{$\mathbf{H F}^{\mathrm{a}}$} \\
\hline$-2 S D$ & $\leq 3.38$ & $66 / 79(84)$ & 104/243 (43) & $5 / 124(4)$ \\
\hline $95 \%$ CI & $\leq 3.42$ & $67 / 79(85)$ & 109/243 (45) & 6/124 (5) \\
\hline 2.3 quantile & $\overline{\leq} 3.28$ & $64 / 79(81)$ & $100 / 243(41)$ & $1 / 124(1)$ \\
\hline \multicolumn{5}{|l|}{ SDNN } \\
\hline$-2 S D$ & $\leq 68$ & $51 / 79(65)$ & 77/243 (32) & 1/123 (1) \\
\hline 95\% CI & $\overline{\leq} 69$ & $51 / 79(65)$ & $79 / 243$ & $2 / 123(2)$ \\
\hline 2.3 quantile & $<78$ & $58 / 79(73)$ & $110 / 243(45)$ & $2 / 123(2)$ \\
\hline \multicolumn{5}{|l|}{ SDANN } \\
\hline -2SD & $\leq 56$ & 43/79 (54) & 66/243 (27) & 1/122 (1) \\
\hline $95 \%$ CI & $\leq 57$ & $43 / 79(54)$ & 69/243 (28) & 1/122 (1) \\
\hline 2.3 quantile & $\leq 65$ & $53 / 79(67)$ & 95/243 (39) & $3 / 122(2)$ \\
\hline \multicolumn{5}{|l|}{ SD } \\
\hline$-2 S D$ & $\leq 28$ & 64/79 (81) & 108/243 (44) & $1 / 123(1)$ \\
\hline $95 \% \mathrm{CI}$ & $\leq 29$ & $66 / 79(84)$ & $114 / 243(47)$ & $1 / 123(1)$ \\
\hline 2.3 quantile & $\leq 30$ & $66 / 79(84)$ & $127 / 243(52)$ & $2 / 123(2)$ \\
\hline
\end{tabular}


Table 23 (continued)

\begin{tabular}{|c|c|c|c|c|}
\hline & & $\begin{array}{c}\text { ESRD with DM } \\
\text { n/ } /(\%)\end{array}$ & $\begin{array}{c}\text { ESRD NonDM } \\
\text { n }(\%)\end{array}$ & $\begin{array}{c}\text { Healthy Controls } \\
\text { n (\%) }\end{array}$ \\
\hline Measure & $\begin{array}{r}\text { Abnormal } \\
\text { Value }\end{array}$ & & & \\
\hline \multicolumn{5}{|l|}{ RMSSD } \\
\hline -2SD & $\leq 7$ & $12 / 79$ (15) & $6 / 243$ (2) & 0/124 (0) \\
\hline 95\% CI & $\leq 7$ & $12 / 79$ (15) & 6/243 (2) & 0/124 (0) \\
\hline 2.3 quantile & $\leq 12$ & $58 / 79(73)$ & $81 / 243(33)$ & $3 / 124(2)$ \\
\hline \multicolumn{5}{|l|}{ PNN50 } \\
\hline -2SD & $\leq-5.5$ & $0(0)$ & $0(0)$ & $0(0)$ \\
\hline $95 \%$ CI & $\leq-5.2$ & $0(0)$ & $0(0)$ & $0(0)$ \\
\hline 2.3 quantile & $\leq 0.3$ & $53 / 78(68)$ & $75 / 242(31)$ & $3 / 124$ (2) \\
\hline \multicolumn{5}{|l|}{ Mean NN } \\
\hline -2SD & $\leq 592$ & 6/79 (8) & $18 / 242(7)$ & 1/123 (1) \\
\hline $95 \% \mathrm{CI}$ & $\overline{<} 595$ & 7/79 (9) & $20 / 242(8)$ & $1 / 123(1)$ \\
\hline 2.3 quantile & $\leq 620$ & $12 / 79(15)$ & $37 / 242(15)$ & 2/123 (2) \\
\hline \multicolumn{5}{|l|}{ ASC Index } \\
\hline$-2 S D$ & $\geq 0.50$ & $37 / 49(76)$ & $86 / 156(55)$ & $0 / 32(0)$ \\
\hline $95 \%$ CI & $\geq 0.53$ & $37 / 49$ (76) & $86 / 156(55)$ & $0 / 32(0)$ \\
\hline 2.3 quantile & $\geq 0.91$ & $37 / 49$ (76) & 76/156 (49) & $1 / 32(3)$ \\
\hline
\end{tabular}

Note. ${ }^{\mathrm{a}}=$ natural $\log (\mathrm{ms} 2)$. The $95 \%$ confidence interval was determined by the formula mean-1.96 (SD). $\mathrm{CI}=$ confidence interval, $\mathrm{ESRD}=$ end-stage renal disease, $\mathrm{DM}=$ diabetes mellitus, NonDM=without diabetes mellitus. HRDB=heart rate change with deep breathing, VR=valsalva ratio, ASC=Autonomic Symptom Checklist. See Table 1 for 24-hr HRV measure definitions. 
Table 24. Normal, Abnormal, and Borderline Values for 2 Evoked Measures, 3 Frequency-Domain Measures, 6 Time-Domain Measures, and the ASC Index Based on the 2.3 and $5^{\text {th }}$ Percentile Values of a Group of Healthy Individuals $(n=158)$

\section{Normal Borderline Abnormal}

\section{Evoked Measures}

HRDB

VR

24-hr Measures

Total power ${ }^{\text {a }}$

$\mathbf{L F}^{\mathbf{a}}$

HF $^{\text {a }}$

SDNN

SDANN

SD

RMSSD

PNN50

Mean NN

ASC Index

$\begin{array}{rrr}\geq 12 & 11 & \leq 10 \\ \geq 1.12 & 1.07-1.11 & \leq 1.06\end{array}$

$\geq 6.11 \quad 5.95-6.10$

$\leq 5.94$

$\geq 5.22 \quad 4.97-5.21 \leq 4.96$

$\geq 3.45 \quad 3.29-3.44 \quad \leq 3.28$

$\geq 85 \quad 79-84 \quad \leq 78$

$\geq 69 \quad 66-68 \leq 65$

$\geq 33 \quad 31-32 \leq 30$

$\geq 14 \quad 13 \leq 12$

$\geq 0.6 \quad 0.4-0.5 \leq 0.3$

$\geq 632 \quad 621-321 \leq 620$

$\leq 0.49 \quad 0.50-0.90 \quad \geq 0.91$

Note. ${ }^{a}=$ natural $\log \left(\mathrm{ms}^{2}\right)$. ESRD=end-stage renal disease, $\mathrm{DM}=$ diabetes mellitus, NonDM=without diabetes mellitus. $\mathrm{HRDB}=$ heart rate change with deep breathing, VR=valsalva ratio, ASC Index=Autonomic Symptom Checklist Index. See Table 1 for 24-hr HRV measure definitions. 


\section{Discussion}

Evoked cardiovascular tests, 24-hr HRV and the ASC index measure objective and subjective autonomic function. The autonomic nervous system is divided into the parasympathetic and sympathetic pathways, which function in an antagonistic manner to maintain the balance of visceral functions and to assure the body responds in an appropriate manner to physical and emotional stressors. Consequences of dysautonomia can affect normal body function, particularly the cardiovascular and gastrointestinal systems, resulting in incapacitating symptoms such as gastroparesis and impotence 6 . Severe autonomic neuropathies which are known to accompany ESRD and diabetes can lead not only to such symptoms, but can result in sudden death $3,12,16,22,59$.

The recognition of morbidity and mortality in patient populations diagnosed with dysautonomia, such as patients with coronary heart disease, ESRD, diabetes mellitus, and sleep disorders, has stimulated technological improvement in diagnostic equipment. New technologies potentially provide earlier diagnosis of dysautonomia. In addition, various disciplines encompassing a multitude of laboratories are examining dysautonomia from different perspectives and increased research is contributing new knowledge. Although standards of measurement for cardiovascular evoked tests have been established, the specific battery of tests used tends to be dependent on the autonomic function laboratory, the population under study, the technology available, and whether the tests are for clinical or research purposes. The establishment of standards for 24-hr HRV measurement and determination of normal referent values is still in an early stage. Thus, it is presently 
recommended that individual laboratories develop their own evoked and 24-hr HRV referent values based on their population of study 4,5 .

When the values characteristic of healthy individuals and ESRD pretransplant patients reported in this study were determined, our findings supported previous data, which suggested that all groups of ESRD patients have severely compromised autonomic function and patients with ESRD and diabetes have the greatest degree of dysautonomia $12,61,93$. The conclusion that the combination of ESRD and diabetes further reduces autonomic function more than ESRD alone is supported. In addition, the ASC Index, which reports an individual's perception of symptoms of dysautonomia and the symptoms impact on the individual's life, indicated increased symptomatology in patients with poorer autonomic function.

When examining the relationship between age and measures of dysautonomia, an inverse relationship was found between most objective measures of dysautonomia and age in healthy individuals, which is congruent with most studies $2,27,31,65$. This relationship was either absent or weaker in the pretransplant ESRD samples. Because HF and LF had a strong inverse correlation with age, it appeared that both sympathetic and parasympathetic function are involved in this relationship. In addition, the frequencydomain demonstrated a stronger correlation with age than the evoked measures supporting the growing consensus that frequency-domain measures are more sensitive to autonomic function than either evoked or time-domain measures. The weaker relationship observed between age and study measures in patients with ESRD was possibly due to the disease itself. These findings are congruent with Bigger et al. ${ }^{2}$ who found a significant decrease in frequency measures with increasing age in healthy adults, 
yet, little association was found between age and HRV in a sample of chronic coronary heart disease patients. However, our study's results, which found that both total power and HF had a strong correlation with age $(\mathrm{r}=-0.60 ; \mathrm{p} \leq 0.003)$ in healthy adults, were in contrast to Bigger et al.'s finding that total power does not significantly decrease with age.

The effect of gender on all values of evoked, 24-hr HRV, and the ASC index for healthy individuals appears to be inconclusive, which is congruent with other studies $2,27,31,65$. In the healthy control group, men had higher values than women, particularly in measures reflective of sympathetic function (LF) and circadian rhythm (SDNN, SDANN). Thus, the effect of gender on measures of dysautonomia may be related to sympathetic function. This pattern was not seen in the ESRD pretransplant samples in contrast to Bigger et al. ${ }^{2}$, who found a gender difference between many $24-\mathrm{hr}$ measures in patients with coronary heart disease. However, the reported values for coronary heart disease patients were not as compromised as those for ESRD pretransplant patients. Thus, it is possible that as values become compromised due to the disease process, the difference between genders observed in healthy individuals becomes attenuated.

Congruent with findings from other studies $2,46,82$, several measures of 24-hr HRV were highly correlated in healthy adults. It has been suggested that when correlations between two variables were this high, the 2 variables can be used interchangeably ${ }^{2}$. Similar patterns of strong correlations among 24-hr heart rate variability measures were seen in the two ESRD pretransplant patient samples. Thus, 
although disease weakens correlations among values, it does not significantly affect them.

Our results established characteristic values for evoked tests and time- and frequency-domain measures of 24-hr HRV for our laboratory, based on a sample of 158 healthy individuals. In general, our values are lower than those reported in the literature $2,27,31,65$, possibly due to our healthy controls being predominately women. Our referent values, consequently, may be conservative. Other factors that could cause variation in referent values among laboratories include analysis techniques, methods of calculation, and differences in geographic populations. For example, the largest variation between laboratory values of healthy adults was noted in total power, which Bigger et al. $2,27,31,65_{\text {measures as }}<0.40 \mathrm{~Hz}$ (total power=9.83), while our laboratory uses 0.01 $1.00 \mathrm{~Hz}$ (total power=7.25). The HF and LF values, which examine the same frequency range as Bigger et al., were much closer (see Tables 15 and 17). Based on the values of the healthy individuals, abnormal values were determined using 2 SDs below the mean, the $95 \%$ confidence interval, and the 2.3 quantile. The abnormal values established using the 2.3 quantile appeared to be the most sensitive. Borderline values were established from the 2.3 quantile to the $5^{\text {th }}$ quantile.

Our findings indicate that the frequency-domain measures are the most sensitive and, thus, the best able to identify patients with dysautonomia. This is congruent with others $2,49,50,109$. For example, when comparing the percentage of abnormal test responses in ESRD DM group to the healthy control group, it was found that total power identified $87 \%$ of the ESRD DM sample as abnormal vs. $3 \%$ of the healthy individuals, LF found $87 \%$ vs. $2 \%$, and HF found $81 \%$ vs. $1 \%$. This is in contrast to Bigger et al. ${ }^{2}$ 
who found that while LF was useful in separating healthy controls from samples of coronary heart disease patients, HF was not useful. The difference in findings could be due to ESRD patients having extremely poor parasympathetic function, which is reflected in the HF. This is important because other studies have established an association between compromised autonomic function $37,38,92$, particularly poor parasympathetic function 93,126 , and sudden death.

In conclusion, this study followed previously made recommendations to establish laboratory and population dependent referent values for measures of autonomic function. Characteristic values for healthy individuals and two samples of ESRD pretransplant patients, in addition to normal, borderline, and abnormal values were determined for each of the study measures. The characteristic values of the two pretransplant ESRD samples were found to be much poorer, especially those of the ESRD with diabetes group, than those for the healthy individual sample. These abnormal values were found to be effective cutpoints for identifying a group with compromised autonomic function in the two patient samples. In addition, the frequency-domain measures were the more sensitive than either the evoked or time-domain measures. The correlation of age with autonomic function measures and the effect of gender were examined and found to be less significant in the patient samples and to vary in importance depending on the study measure. This was thought to be due to the disease process attenuating the effect of age and gender on autonomic function. The disease process had a minimal effect on correlations among time- and frequency-domain variables. 


\section{Chapter 4. Correlation and Sensitivity Among Three Instruments Designed to Measure Autonomic Function}

Altered cardiovascular autonomic function is a frequent complication of end-stage renal disease (ESRD) causing a myriad of clinical symptoms ranging from gastroparesis and impotence to cardiac death 12,61 . It is of particular concern for patients with ESRD and diabetes mellitus (DM) with $50 \%$ of this population dying within two years of initiating dialysis therapy ${ }^{1}$. Although, no universal gold-standard or battery of tests exists to evaluate autonomic function 45 , several objective methods have been described and compared as to sensitivity in the ESRD population. Two such methods are evoked cardiovascular measures and 24-hr heart rate variability (HRV) measures. While dysautonomia has been found to be relatively common in newly diagnosed type 1 diabetes mellitus patients 35 when assessed with objective measures of autonomic function, symptoms of dysautonomia are not common and are thought to occur late in the disease process 59, 61. Our laboratory uses two objective tests, evoked and 24-hr HRV, in addition to symptomatology to evaluate dysautonomia in ESRD patients awaiting kidney or kidney-pancreas transplantation. A scoring system that includes all three tests would be useful in describing the severity of the individual's dysautonomia, in addition to allowing a quantitative way to monitor the progression of the disease and interventions designed to improve autonomic function 127 . However, studies have not defined the relationships among these three measures of dysautonomia. In addition, it is unknown to what extent early abnormalities in objective measures of autonomic function can predict 
the onset and progression of clinical symptoms ${ }^{4}$. The purpose of this study was to compare and contrast three different instruments that assess autonomic function. In addition, we sought to establish correlations between physiologic measures of dysautonomia and symptoms as a step toward defining a threshold where physiologic measures herald the occurrence of symptomatology. To this purpose, the following research aims and questions were investigated.

1. Determine the relationships of measures within the evoked tests, 24-hr heart rate variability tests, and the Autonomic Symptom Checklist.

1a) What are the correlations among measures of evoked cardiovascular tests?

1b) What are the correlations among measures of 24-hr heart rate variability?

1c) What are the correlations among measures within sections of the Autonomic Symptom Checklist?

2. Compare the clinical utility of the evoked tests, 24-hr heart rate variability monitoring, and the Autonomic Symptom Checklist.

2a) What are the correlations among the evoked scores, the time- and frequencydomain scores of 24-hr HRV, the 24-hr heart rate variability scores, and the Autonomic Symptom Checklist scores?

2b) What are the sensitivities for detecting dysautonomia of the evoked scores, 24-hr heart rate variability scores, and the Autonomic Symptom Checklist scores? 


\section{Introduction}

Dysautonomia is difficult to measure and quantify due to the dual innervation of the ANS (sympathetic and parasympathetic pathways), the numerous reflex arcs involved, and the anatomic dispersion of autonomic nerve fibers ${ }^{14}$. Even with these measurement obstacles, quantification of dysautonomia using objective physiologic measures of autonomic function has been reported in the literature since the 1950 's $3,15-17$. Prior to that time, there were occasional references to clinical signs and symptoms thought to indicate the presence of autonomic neuropathies ${ }^{18}$. In the 1970 s, investigators began using a battery of bedside autonomic function tests to aid in interpretation of these vague symptoms in both diabetic ${ }^{19}$ and uremic 20 patients. A battery of tests has been recommended $3,21,22$ because researchers have been unable to isolate a single best measure of autonomic function. This battery of tests has moved from the bedside to the laboratory and has led to improved diagnosis and treatment of autonomic neuropathies 3 .

\section{Evoked Cardiovascular Measures}

Two evoked measures commonly included in a battery of tests designed to evaluate autonomic function are the change in heart rate with deep breathing (HRDB) and the Valsalva ratio $(\mathrm{VR})^{3}, 20,22$. Both measures assess a complex reflex arc reflecting a "beat to beat" balance of sympathetic and parasympathetic activity which can

easily be altered by physical and/or emotional stress ${ }^{3}$. Numerous studies $3,20,22-32,41$ have used both HRDB and VR to evaluate cardiac autonomic function in normal, uremic, 
diabetic, and transplant populations. The high reliability, ease of administration, and non-invasive nature of these tests make them the most popular investigative tools 3 . However, there are two major limitations to evoked measures. First, the patient must be willing and able to cooperate with the protocol. Second, although a procedure is standardized, stimuli could evoke different mechanisms in different individuals, thus, the resulting measure may not always reflect the same dysfunction 33 .

Although the HRDB has been shown to be the single most sensitive evoked measure in many studies $22,25,63$, the VR has been shown to be the most sensitive in studies of posttransplant kidney and kidney-pancreas patients 99 and newly diagnosed diabetic patients 35 . In addition, one study 32 observed that some patients had a markedly reduced HRDB with a paradoxically normal VR. When explored further, the investigators determined that attenuation of both measures indicated possible additional impairment of cardiac adrenergic failure, in addition to the already suspected parasympathetic failure. Thus, there is a lack of consensus concerning which of these two measures is the most sensitive. However, when assessing autonomic function with only evoked measures it is thought that, once the measure becomes abnormal, subjects either remain static or deteriorate ${ }^{3}$. 


\section{4-hr Heart Rate Variability Measures}

Twenty-four hour monitoring of heart rate variability and use of power spectral analysis has gained interest as an alternative method to evoked tests for evaluating autonomic function. This method was first appreciated clinically as a strong and independent predictor of mortality after an acute myocardial infarction 37,122 . Heart rate variability has the potential to assess earlier stages of autonomic cardiovascular dysfunction, in addition to examining circadian rhythmicity $42,45-47$. Test measures are frequently reported in both the time- and frequency-domains (see Table 1). In an early study using 24-hr HRV measures, Ewing, Neilson, and Travis 48 assessed cardiac parasympathetic function using the PNN50 derived from 24-hr electrocardiograms obtained from healthy subjects and diabetic patients. The results showed the 24-hr HRV method was better than evoked tests in detecting early cardiac parasympathetic damage. In another study, Comi, Sora, Bianchi, Bontempi, Gianoglio, Cerutti, Micossi, and Canal ${ }^{49}$ compared abnormalities in autonomic function found with HRV recordings to abnormalities identified with a battery of evoked tests and found that one-fourth of the diabetic patients with normal evoked measures had abnormal power spectral analysis results. Thus, researchers using HRV measures demonstrated this technique as a more sensitive indicator of dysautonomia than evoked measures. In addition, power spectral analysis allowed better quantification of autonomic function into parasympathetic and sympathetic function. Using HRV, research of diabetic $49-51$ and uremic ${ }^{52}$ patients is dispelling the previously held belief that diabetic autonomic neuropathy primarily affects 
parasympathetic function. These studies report lower values in measures of sympathetic activity, as well as parasympathetic activity.

Several 24-hr HRV time- and frequency-domain measures are strongly correlated with each other 46,82 . It is thought that these strong correlations exist because of both mathematical and physiological relationships among time- and frequency-domain measures 53 . Kleiger et al. 46 obtained 24-hr HRV measures from 14 healthy individuals (20-55 years of age) and found that certain time- and frequency-domain variables were highly correlated and concluded that the measures may serve as surrogates for each other. High frequency power, RMSSD, and PNN50, all considered measures of vagal function, were strongly correlated $(r>0.9)$ with each other and with SD. Low frequency power was also strongly correlated with the measures of vagal function $(r>0.8)$ indicating that some parasympathetic activity is reflected in low frequency power.

Bigger et al. 82 examined the correlations between time- and frequency-domain measures in 715, 24-hr HRV recordings obtained two weeks after myocardial infarction. They found natural groupings for several of the 24-hr measures. From Bigger et al.'s data, Keehn 83 deduced that both SDNN and SDANN are essentially equivalent to total power (see Table 1 for measure definitions). It was suggested 82 that these measures could act as surrogates for each other. Similar patterns of correlation among measures of parasympathetic function (RMSSD, PNN50, and HF) were reported in an earlier study 84 and in a later, larger study ${ }^{2}$. In addition, the SDNN and the square root of total power were almost perfectly correlated, and the time-domain measure, SDNN, was found strongly and significantly associated with mortality in this group 2,37 . 


\section{Symptomatology}

Historically, the diagnosis of autonomic neuropathy was based primarily on clinical features including postural hypotension, intermittent diarrhea, hypoglycemic unawareness, sweating abnormalities, gastric fullness, and impotence ${ }^{18,59}$, which generally occur late in the disease process. Symptoms of autonomic dysfunction were initially described by Rundles 60 in 1945 . At that time, limited treatment options existed for patients with uremia with or without diabetes, and complications resulting from these diseases generally caused a shortened life expectancy. Patients did not always live long enough to manifest symptoms that occurred late in the disease process and symptoms of dysautonomia were thought uncommon. Improved medical treatment options have lengthened a patient's life expectancy, resulting in increased reporting of symptomatology. MacKay et al. 63 reported that, although diabetics with autonomic symptoms as a rule also had abnormal evoked tests, about one-third of the diabetics without symptoms also had abnormal evoked tests. Symptoms were, therefore, an insensitive measure of dysautonomia when compared to evoked measures 20,63 and 24hr HRV measures 50 . In addition, symptoms of dysautonomia, particularly orthostatic hypotension, gastric problems and hypoglycemia unawareness, in combination with abnormal evoked measures of autonomic function, carry a very poor prognosis 61 . It is unknown, if once a patient's autonomic function is allowed to deteriorate to the point of symptomatology, whether an intervention such as transplantation can improve the dysautonomia enough to ameliorate the symptoms. The purpose of this study was to examine the relationships among two objective and one subjective test of autonomic function in ESRD patients both pre and post kidney or kidney-pancreas transplant. 


\section{Methodology}

\section{Sample}

A convenience sample of patients with ESRD pre-kidney or kidney-pancreas transplant or posttransplant $(\mathrm{n}=185)$ were evaluated for dysautonomia between February 1996 through February 1998. All data obtained were a part of the patient's normally scheduled evaluation. All transplant patients over 18 years of age were included in the study unless they were physically unable to participate in data collection or if they were found to have other neurologic disorders which could affect autonomic function. Each patient with diabetes mellitus had blood glucose levels checked prior to autonomic function testing. If the blood glucose was $200 \mathrm{mg} / \mathrm{dl}$ or over the testing was cancelled and rescheduled. Demographic data included information on current medications, alcohol and tobacco use. In addition, a group of healthy persons $(n=22)$ was recruited from faculty, family and friends to determine normal values for autonomic function in our Physiologic Function Laboratory and for comparison with the ESRD patients. Healthy control data were collected between November 1995 through February 1998. Subjects were considered healthy if they reported no neurologic dysfunction or coronary heart disease. This study was approved by the Institutional Review Board of the University of Tennessee, Memphis. Each participant signed an informed consent (see Appendix B). All participants completed all evoked and 24-hr HRV tests, and the Autonomic Symptom Checklist (ASC). 


\section{Procedure}

Study participants were recruited, the study explained, and those volunteering scheduled for an appointment in the Physiologic Function Lab. On the day of testing a consent form was signed, demographic data obtained, evoked cardiovascular tests done, ASC completed, and an ambulatory Holter monitor applied. Participants were asked to return the Holter monitor within 36 to 48 hours. Following data collection, analysis was completed and reviewed weekly.

\section{Evoked Cardiovascular Test}

Evoked measures, change in heart rate with deep breathing (HRDB) and the Valsalva ratio (VR) were obtained by tests performed in a temperature-controlled laboratory dedicated to performing autonomic function tests . Environmental stimuli such as noise and light were minimized. A Power Macintosh 9500 computer system and AcqKnowledge ${ }^{\circledR}$ III BIOPAC software were used for data acquisition and analysis of standard electrocardiogram recordings and blood pressure measurements obtained from a Dinamap $^{\mathrm{TM}}$ XL (CRITIKON). The protocols (see Appendix A) used to perform the evoked tests were modified from Mayo Clinic's protocols 73 , and a brief overview follows.

The HRDB value was obtained while the patient was relaxed in a recumbent position breathing deeply and regularly for one minute at a rate of 6 breaths per minute ( 5 second inhale/5 second exhale) which follows a two minute period of regular breathing. The difference between the maximum and minimum heart rates was calculated. The second test, the VR, was derived by dividing the maximum heart rate during a seated patient's forced expiration of $40-\mathrm{mm} \mathrm{Hg}$ for 15 seconds by the minimum heart rate within 
15 seconds after the maneuver (see Appendix A). Reliability and validity have been established for HRDB and $\mathrm{VR}^{5}, 19,23,63$.

\section{Ambulatory 24-hr Holter Recordings}

Data collection and processing of 24-hr HRV measures followed manufacturer recommendations 83 in addition to being modified from recommendations by the Task Force of the European Society of Cardiology and the North American Society of Pacing and Electrophysiology 125 . It is briefly summarized here. Analysis of 24-hr ambulatory Holter monitor tapes was completed by Marquette Electronics Laser SXP® Ambulatory ECG Analysis and Editing Systems with version 5.8 software program and Series 8500 Holter recording system. Each QRS complex was digitized, identified and labeled. The analyzed data file was then scanned and manually edited to locate and correct any errors in QRS labeling that would adversely affect measurement of heart rate variability. Tapes were required to have $\geq 20$ hours of analyzable data and generally had $\geq 23$ hours of analyzable data. Using these data files, 24-hr HRV with power spectral analysis was calculated (see Appendix A).

Recently, it was recommended that four measures be used for time-domain HRV assessment; SDNN, HRV triangular index, SDANN, and RMSSD 53 . Because both the SDNN and the HRV triangular index are estimates of overall HRV 53 , and the SDNN is more routinely reported in the literature 37 , this series of studies will report SDNN in place of HRV triangular index. In addition, this series of studies is part of a longitudinal research protocol funded by the National Institute of Health and is in compliance with the study measures as defined there 76 . 
The time-domain HRV calculations are placed in two general categories (see Table 1). The first category is derived directly from the R-R intervals and includes means and standard deviations of the interval. Measures in this category of the timedomain include the SDNN and the SDANN. The SDNN is the standard deviation of all $\mathrm{R}-\mathrm{R}$ intervals during the 24-hours and has been found to be associated with sudden cardiac death ${ }^{37}$. The SDANN is the standard deviation of the means of R-R intervals found in successive five minute blocks over 24-hours and is considered the best measure of overall autonomic balance and represents circadian rhythmicity of autonomic function ${ }^{75}$, in addition to estimating long-term components of HRV 125 . The second category of time-domain variables is based on the differences between adjacent $\mathrm{R}-\mathrm{R}$ intervals and included the RMSSD. The RMSSD is the square root of the mean of the sum of squares of differences between adjacent R-R intervals and is virtually independent of circadian rhythms. It reflects alterations in autonomic function that are primarily vagally mediated 57,125 (see Table 1).

The frequency-domain measures are calculated by fast Fourier transform which indicates the relative amount of total $(0.01-1.00 \mathrm{~Hz})$, low $(0.04-0.15 \mathrm{~Hz})$ and high $(0.15-$ $0.40 \mathrm{~Hz}$ ) frequency power ${ }^{83}$. Low frequency waveforms estimates sympathetic activity along with some parasympathetic activity, while high frequency waveforms, also known as the respiratory frequency, represents parasympathetic activity 9,10 (see Table 1).

Quality assurance data provided by the manufacturer illustrates mathematically correct results following submission of known electronically generated cardiac signals 83 . This technology has been successfully employed in clinical studies evaluating alterations in autonomic regulatory mechanisms in patients with heart disease ${ }^{38}$, cardiac 
transplantation 85 , diabetic autonomic neuropathy $42,48,86$ uremia $^{52,87}$ and kidney transplantation 88 . Twenty-four hour measures are highly stable in both normal subjects $^{46}$, post myocardial infarction patients 55 , and patients with ventricular arrhythmias 89 . Thus, it is thought that 24-hr HRV measures may be ideal for assessing intervention therapies ${ }^{53}$, such as transplantation ${ }^{110}$, exercise 68 , and deep breathing relaxation techniques 113 .

\section{Autonomic Symptom Checklist}

The Autonomic Symptom Checklist (ASC) is a self-administered checklist developed using a focus group approach and designed to recognize the perceived presence, distribution and severity of autonomic symptoms (see Appendix C). Development and psychometric testing have been previously described (Chapter 2), and will be summarized here. The ASC assesses dysautonomia symptoms in 5 categories with 32 questions, each using a 7-point Likert scale. Four additional questions address daily life-style habits that may influence autonomic function. The five categories of symptoms are postural hypotension, sudomotor abnormalities, gastrointestinal problems, impotence, and hypoglycemia unawareness. A question is also asked in each category to obtain a score reflecting patients' perceptions of how problematic symptoms are.

Validity of the ASC was established by administering it to two groups of ESRD patients with known autonomic dysfunction, diabetic ESRD patients $(n=85)$ and nondiabetic ESRD patients $(n=159)$ and to healthy controls $(n=35)$. The DM group reported the greatest symptomatology, followed by the NonDM group, and with least symptoms, the control group. This pattern was present for all symptom categories. 
There was significant difference $(\mathrm{p} \leq 0.0001)$ among all groups for three symptom scores (orthostatic hypotension, gastrointestinal problems, and impotence) and two impact scores (gastrointestinal problems and impotence). There was significant difference ( $\mathrm{p} \leq$ 0.003) between healthy controls and both ESRD groups for one symptom score (sudomotor abnormalities) and two impact scores (orthostatic hypotension and sudomotor abnormalities. In addition, findings demonstrated a significant difference $(\mathrm{p} \leq 0.03)$ in symptoms between patients with diabetes who were hypoglycemia aware and hypoglycemia unaware. Test-retest reliability for subscales yielded moderate to high rvalues (0.59 to 1.00), except for the sudomotor subscale. The ASC appropriately differentiated among control and two groups with known autonomic dysfunction. In addition, the diabetes mellitus groups, which have been shown to have the poorest objective measures of autonomic function, also had the highest symptom scores. Thus, increased symptomatology is seen in patients with worsening physiologic autonomic function.

\section{Data Analysis}

Only pretransplant and posttransplant patients who had values for all study measures (evoked, 24-hr HRV, and ASC ) were included in data analysis. Pretransplant patients with type 2 diabetes mellitus were excluded from analysis, thus, the ESRD with DM group was composed of patients with only type 1 diabetes mellitus. This prevented any confounding effects due to physiologic differences that might occur by including both type 1 and type 2 diabetes mellitus patients in one group. Chi square tests were used to determine equivalence of groups for categorical data. One-way ANOVA with preplanned multiple comparisons using Least Square Means was used to test for 
differences among study groups. Correlational analysis was used to evaluate the relationship between study measures and time- and frequency-domain measures.

Each individual's HRDB and VR were assigned a value of 1 (normal), 2 (borderline), or 3 (abnormal) based on our lab's referent values (see Table 24) determined by healthy individuals $(n=158)$. These values were then summed and averaged to calculate the Evoked Score. Each of the time- and frequency-domain measures were assigned a value of 1 (normal), 2 (borderline), or 3 (abnormal) based on our lab's referent values (see Table 24) determined by healthy individuals $(n=158)$ and reported in Chapter 3. These values were then summed and averaged to calculate the 24-hr HRV score. To allow comparisons between sensitivity of the time- and frequency-domain measures, an individual time-domain score and an individual frequency-domain score were calculated by summing and averaging the values assigned to their respective measures. Thus, time-domain score elements were SDANN, SDNN and RMSSD; frequency-domain score elements were total power, HF, and LF; and 24-hr HRV score elements were SDANN, SDNN, RMSSD, total power, HF, and LF. Each score ranged from 1 to 3 .

Several scoring systems have been suggested to grade the severity of autonomic damage $3,41,127$. However, not all scoring systems account for borderline values and not all evaluate the same study measures we have assessed. Thus, our scoring system is modified from others and based on our experience with autonomic measurement in ESRD patients. Our scoring system was developed by assessing for cutpoints in the data. Cutpoints were established for each score such that if the majority of values determining that score were normal, then the score was normal. If the values were equally normal and 
borderline, or there were more borderline values than abnormal values the score was determined to be borderline. If the values were equally borderline and abnormal, or if there were more abnormal values, then the score was determined to be abnormal. The only exception was the evoked score where if both values were normal, then the score was normal and if both values were abnormal then the score was abnormal. All other evoked values were determined to be borderline. Thus, using this modified scoring system, each of the five scores ranged from 1-3 with normal defined as 1.0-1.4, borderline defined as 1.5-2.4, and abnormal defined as 2.5 to 3.0.

\section{Results}

The evoked measures, 24-hr HRV measures, and all sections of the ASC for a group of ESRD pre and posttransplant individuals and healthy controls $(n=290)$ were visually inspected for incomplete data sets. Fifty-three individuals were eliminated from the study due to missing values, which left a sample size of 237 subjects. Only one set of tests measures was used for each individual, thus, if an ESRD transplant patient had both pretransplant and posttransplant data, the pretransplant data $(n=29)$ were eliminated from the study sample. Including the posttransplant data, in addition to having healthy controls in the study sample, increased the variability among values. The final study sample $(n=208)$ was composed of pretransplant ESRD patients $(n=130)$, posttransplant patients $(n=55)$, and healthy individuals $(n=22)$. The total sample had a mean age of $40.2 \pm 10.4$, with $84(41 \%)$ women and 88 (43\%) African-Americans. 


\section{Correlations of Within Test Measures}

A correlational analysis among the two evoked measures, HRDB and VR, showed them to be strongly correlated $(\mathrm{r}=0.63 ; \mathrm{p} \leq 0.00)$ in this sample of healthy individuals, pre and posttransplant patients. Correlations among the six time- and frequency-domain measures were also strong $(\mathrm{p} \leq 0.00)$ with all $\mathrm{r}$-values $\geq 0.67$ (see Table 25$)$. Correlations were strongest $(\mathrm{p} \leq 0.00)$ among the frequency-domain measures, especially between total power and HF (r=0.90) and total power and LF (r=0.99). In addition, two timedomain measures, SDANN and the SDNN, were highly correlated ( $r=0.99 ; \mathrm{p} \leq 0.00)$. The SDNN and the SDANN were correlated $(\mathrm{p} \leq 0.00)$ to total power at $\mathrm{r}$-values of 0.79 and 0.71 , respectively. The SDANN had the lowest correlation with frequency-domain measures (LF, r=0.69; HF, r=0.67), however, they were still strongly correlated $(\mathrm{p} \leq$ 0.00). Data in Table 26 displayed the results of a correlational analysis examining the relationship among the ASC symptom and impact scores for the three categories completed by the total sample. All symptom scores were positively correlated with all other symptom and impact scores $(\mathrm{r}=0.27$ to $0.81 ; \mathrm{p} \leq 0.00)$. Correlations between individual category symptom scores and their respective impact scores were high $(\mathrm{r} \geq$ 0.74; $\mathrm{p} \leq 0.00$ ), with the highest correlation occurring between Orthostatic Hypotension symptom and impact scores $(\mathrm{r}=0.81 ; \mathrm{p} \leq 0.00)$. The lowest correlation $(\mathrm{r}=0.27 ; \mathrm{p} \leq 0.00)$ was seen between Sudomotor impact and Gastrointestinal symptoms. A correlational analysis was completed on a male subgroup $(n=123)$ examining the impact of the Impotence category on correlations (see Table 27). All symptom and impact scores remained positively correlated with $r$-values ranging from $0.22 ; \mathrm{p} \leq 0.01$ (Impotence 
Table 25. Correlational Analysis Among Six Time- and Frequency-Domain Measures of 24-hr HRV in ESRD Pre and Posttransplant $(n=185)$ and Healthy Individuals $(\mathbf{n}=\mathbf{2 2})$

\begin{tabular}{|c|c|c|c|c|c|c|}
\hline & \multicolumn{3}{|c|}{ Frequency Measures } & \multicolumn{3}{|c|}{ Time Measures } \\
\hline & TotPower & LF & $\mathrm{HF}$ & SDNN & SDANN & RMSSD \\
\hline TotPower & 1.00 & & & & & \\
\hline $\mathrm{LF}$ & 0.99 & 1.00 & & & & \\
\hline $\mathrm{HF}$ & 0.90 & 0.88 & 1.00 & & & \\
\hline SDNN & 0.79 & 0.77 & 0.74 & 1.00 & & \\
\hline SDANN & 0.71 & 0.69 & 0.67 & 0.99 & 1.00 & \\
\hline RMMSD & 0.76 & 0.71 & 0.88 & 0.69 & 0.62 & 0.89 \\
\hline
\end{tabular}

Note. TotPower=Total Power, LF=low frequency, HF=high frequency, See Table 1 for definitions of measures. 
Table 26. Correlational Analysis Among Measures in Three Sections of the ASC in Pre and Posttransplant $(n=185)$ and Healthy Individuals $(n=22)$

\begin{tabular}{|c|c|c|c|c|c|c|}
\hline & \multicolumn{3}{|c|}{ Symptom Scores } & \multicolumn{3}{|c|}{ Impact Scores } \\
\hline & Ortho & Sudo & GI & Ortho & Sudo & GI \\
\hline \multicolumn{7}{|c|}{ Symptom Scores } \\
\hline Ortho & 1.00 & & & & & \\
\hline Sudo & 0.41 & 1.00 & & & & \\
\hline GI & 0.55 & 0.34 & 1.00 & & & \\
\hline \multicolumn{7}{|c|}{ Impact Scores } \\
\hline Ortho & 0.81 & 0.32 & 0.53 & 1.00 & & \\
\hline Sudo & 0.38 & 0.74 & 0.27 & 0.36 & 1.00 & \\
\hline GI & 0.49 & 0.30 & 0.79 & 0.54 & 0.32 & 1.00 \\
\hline
\end{tabular}

Note. Ortho=orthostatic, Sudo=sudomotor, GI=gastrointestinal 
symptoms and Sudomotor Impact) to $0.81 ; \mathrm{p} \leq 0.00$ (Orthostatic Hypotension symptoms and impact). Impotence symptom and impact scores were moderately correlated ( $\mathrm{p} \leq$ 0.01 to 0.00 ) with most other symptom and impact values ( $r=0.25$ to 0.56 ). A correlational analysis examining the Hypoglycemia Unaware category (see Table 28) was completed on a subgroup of ESRD patients with diabetes mellitus $(n=30)$. The results showed that all correlations were positive, ranging from $\mathrm{r}=0.29 ; \mathrm{p} \leq 0.12$ (Sudomotor impact and Hypoglycemia Unaware symptom) to $r=0.87 ; \mathrm{p} \leq 0.00$ (Orthostatic symptoms and impact). These results concur with the high r-values between Orthostatic symptom and impact values that were found for the total sample of pre and posttransplant ESRD patients and healthy individuals and for men subgroup sample.

\section{Clinical Utility of Measures of Autonomic Function}

To compare the evoked tests, time- and frequency-domain tests, and the ASC test each measure within each test was assigned a value of 1 (normal), 2 (borderline) or 3 (abnormal) and a score was obtained as previously described. To examine the sensitivity of the five scores (evoked, time, frequency, 24-hr HRV, and ASC) the sample was divided into three groups; healthy individuals $(\mathrm{n}=22)$, pretransplant patients $(\mathrm{n}=130)$, and posttransplant patients $(n=55)$. Data from the three groups were examined to determine the frequency of normal (1-1.4), borderline (1.5-2.4), and abnormal (2.5-3) scores for each of the five reported study scores (evoked, time, frequency, 24-hr HRV, and ASC) as previously described. Characteristics of the three groups are displayed in Table 29. There were no significant differences in age or gender among the three groups. The groups were significantly 
Table 25. Correlational Analysis Among Measures in Four Sections of the ASC in Pre and Posttransplant ESRD and Healthy $\operatorname{Men}(n=123)$

Symptom Scores

\begin{tabular}{|c|c|c|c|c|c|c|c|c|}
\hline & Orthostatic & Sudomotor & GI & Impotence & Orthostatic & Sudomotor & GI & Impotence \\
\hline \multicolumn{9}{|l|}{ Symptom Scores } \\
\hline Orthostatic & 1.00 & & & & & & & \\
\hline Sudomotor & 0.46 & 1.00 & & & & & & \\
\hline Gastrointestinal & 0.56 & 0.43 & 1.00 & & & & & \\
\hline Impotence & 0.37 & 0.25 & 0.40 & 1.00 & & & & \\
\hline \multicolumn{9}{|l|}{ Impact Scores } \\
\hline Orthostatic & 0.81 & 0.39 & 0.60 & 0.33 & 1.00 & & & \\
\hline Sudomotor & 0.52 & 0.73 & 0.37 & 0.22 & 0.51 & 1.00 & & \\
\hline Gastrointestinal & 0.51 & 0.41 & 0.77 & 0.32 & 0.61 & 0.47 & 1.00 & \\
\hline Impotence & 0.47 & 0.35 & 0.43 & 0.56 & 0.49 & 0.47 & 0.39 & 1.00 \\
\hline
\end{tabular}


Table 26. Correlational Analysis Among Measures in Four Sections of the ASC in Pre and Posttransplant ESRD Patients with Diabetes Mellitus (n= 51)

Symptom Scores $\quad$ Impact Scores

\begin{tabular}{|c|c|c|c|c|c|c|c|c|}
\hline & Orthostatic & Sudomotor & GI & $\mathrm{HGU}$ & Orthostatic & Sudomotor & GI & HGU \\
\hline \multicolumn{9}{|l|}{ Symptom Scores } \\
\hline Orthostatic & 1.00 & & & & & & & \\
\hline Sudomotor & 0.75 & 1.00 & & & & & & \\
\hline Gastrointestinal & 0.60 & 0.53 & 1.00 & & & & & \\
\hline $\mathrm{HGU}$ & 0.59 & 0.45 & 0.49 & 1.00 & & & & \\
\hline \multicolumn{9}{|l|}{ Impact Scores } \\
\hline Orthostatic & 0.87 & 0.67 & 0.50 & 0.48 & 1.00 & & & \\
\hline Sudomotor & 0.63 & 0.79 & 0.45 & 0.29 & 0.53 & 1.00 & & \\
\hline Gastrointestinal & 0.46 & 0.55 & 0.78 & 0.35 & 0.39 & 0.37 & 1.00 & \\
\hline HGU & 0.51 & 0.51 & 0.44 & 0.74 & 0.49 & 0.36 & 0.44 & 1.00 \\
\hline
\end{tabular}

Note. Orthostatic=orthostatic hypotension, GI=gastrointestinal, HGU=hypoglycemiả unawareness.

$\bar{\infty}_{\infty}$ 
different when compared by percentage of African-Americans (Healthy individuals $=0 \%$, PreTx $=55 \%$, PostTx $=31 \% ; \mathrm{p} \leq 0.001)$. Because diabetes in combination with uremia results in further autonomic function deterioration, the groups were compared by diabetic status prior to transplantation and found to be significantly different (PreTx $=23 \%$, $\operatorname{PostT} x=38 \% ; \mathrm{p} \leq 0.03)$.

Table 30 displays the correlational analysis among the test scores. All scores were positively and strongly correlated. It was expected that the time- and frequencydomain scores would be highly correlated $(\mathrm{p} \leq 0.00)$ to the $24-\mathrm{hr}$ HRV score $(r=0.94$, $\mathrm{r}=0.95$; respectively) because they were the two components of the 24-hr HRV score. However, the time- and frequency-domain scores were also strongly correlated $(\mathrm{r}=0.78$; $p \leq 0.00)$ to each other. The evoked score was also strongly correlated $(p \leq 0.00)$ with the time-domain score, frequency-domain score, and the 24-hr HRV score ( $\mathrm{r}=0.55,0.63$, 0.63; respectively). The ASC score had only weak to moderate correlations $(\mathrm{p} \leq 0.01)$ with all other scores ( $r=0.18$ to 0.29$)$.

The results of examining the sensitivity of the five measures are displayed in Table 31. As the results show, the frequency-domain score found $38 \%$ of the pretransplant patients and $42 \%$ of the posttransplant patients to have abnormal scores. Compared to the time-domain, which indicated $30 \%$ of the pretransplant patients and $29 \%$ posttransplant patients abnormal, the frequency-domain was a more sensitive indicator of dysautonomia. The ASC score, in addition, was sensitive in identifying 
Table 27. Characteristics of Healthy Individuals, ESRD Pretransplant Patients, and ESRD Posttransplant Patients

\begin{tabular}{|c|c|c|c|c|}
\hline & & $\begin{array}{c}\text { Healthy } \\
\text { Individuals } \\
(\mathbf{n}=22)\end{array}$ & $\begin{array}{c}\text { ESRD Pretransplant } \\
\text { Patients } \\
(\mathbf{n}=130)\end{array}$ & $\begin{array}{c}\text { Posttranspla } \\
\text { Patients } \\
(\mathbf{n}=\mathbf{5 5})\end{array}$ \\
\hline \multicolumn{5}{|l|}{ Characteristic } \\
\hline \multirow[t]{2}{*}{ Age (yrs.) } & mean \pm SD & $40.7 \pm 8.1$ & $40.6^{ \pm} 10.9$ & $39.0 \pm 10.0$ \\
\hline & range & $25-55$ & $19-67$ & $20-66$ \\
\hline \multicolumn{5}{|l|}{ Gender } \\
\hline Men & $\mathrm{n}(\%)$ & $15(68)$ & 77 (59) & $31(56)$ \\
\hline Women & $\mathrm{n}(\%)$ & $7(32)$ & $53(41)$ & $24(44)$ \\
\hline \multicolumn{5}{|l|}{$\begin{array}{l}\text { Pretransplant } \\
\text { Diabetic Status }\end{array}$} \\
\hline DM & $\mathrm{n}(\%)$ & na & $30(23 \%) \ddagger$ & $21(38 \%) \ddagger$ \\
\hline NonDM & $\mathrm{n}(\%)$ & na & $100(77 \%) \ddagger$ & $34(62 \%) \neq$ \\
\hline \multicolumn{5}{|l|}{ Race } \\
\hline African-Amer. & $\mathrm{n}(\%)$ & $0^{*}$ & $71(55)^{*}$ & $17(31)^{*}$ \\
\hline Caucasian & $\mathrm{n}(\%)$ & $22(100)^{*}$ & $59(45)^{*}$ & $37(67)^{*}$ \\
\hline Other & $\mathrm{n}(\%)$ & 0 & 0 & 1 (2) \\
\hline
\end{tabular}

Note. ${ }^{*} \mathrm{p} \leq 0.001 \neq \mathrm{p} \leq 0.03$ between groups with like symbols. 
symptoms of dysautonomia (as measured by abnormal scores) in ESRD pretransplant $(59 \%)$ and posttransplant (58\%) patients, which are known to have poor autonomic function. The evoked score was found to be the least sensitive measure of dysautonomia as compared to the time- or frequency-domain scores, with findings reporting only $24 \%$ abnormal scores in the pretransplant sample and $25 \%$ abnormal scores in the posttransplant sample. There was little difference in scores between the ESRD pretransplant and posttransplant samples.

\section{Discussion}

Dysautonomia is a well-known complication of ESRD and diabetes ${ }^{3}$ and contributes significantly to its mortality and morbidity ${ }^{1}$. Research suggesting various treatment modalities to prevent diabetic autonomic neuropathy has been reported 108 . However, it appears that the onset of dysautonomia at a sub-clinical level is already present at the time of diagnosis 35 for some patients with diabetes. It is critical that clinicians have access to sensitive, reliable, noninvasive, and quantitative means of identifying patients at high risk of developing dysautonomia. Then, if treatment modalities such as exercise and relaxation are found that can alter the progression of dysautonomia 113 , they can be applied as early as possible.

Abnormalities detected by 24-hr HRV monitoring are believed to be more sensitive indicators of autonomic dysfunction than evoked tests, particularly in patients with diabetes who have significantly less 24 -hour R-R interval variability 48 . Our findings support this, however, we also found that while the time-domain scores were sensitive indicators of dysautonomia, the frequency-domain scores were more 
Table 28. Correlational Analysis Among Evoked Score, Time-Domain Score, Frequency Score, 24-hr HRV Score, and ASC Score in ESRD Pre and

Posttransplant $(n=185)$ and Healthy Individuals $(n=22)$

\begin{tabular}{|c|c|c|c|c|c|}
\hline & Evoked Score & Time Score & Frequency Score & 24-hr HRV Score & ASC Score \\
\hline Evoked Score & 1.00 & & & & \\
\hline Time-Domain Score & 0.55 & 1.00 & & & \\
\hline Frequency-Domain Score & 0.63 & 0.78 & 1.00 & & \\
\hline 24-hr HRV Score & 0.63 & 0.94 & 0.95 & 1.00 & \\
\hline ASC Score & 0.18 & 0.30 & 0.25 & 0.29 & 1.00 \\
\hline
\end{tabular}

Note. See Table 1 for definitions of measures. 
Table 29. Sensitivity of Evoked, Time, Frequency, 24-hr HRV and ASC Scores in Healthy Individuals, ESRD Pretransplant Patients, and Posttransplant Patients

\begin{tabular}{|c|c|c|c|}
\hline Test Measures & $\begin{array}{c}\text { Healthy } \\
\text { Individuals } \\
(\mathrm{n}=22)\end{array}$ & $\begin{array}{c}\text { ESRD } \\
\text { Pretransplant } \\
\text { Patients } \\
(n=130)\end{array}$ & $\begin{array}{c}\text { Posttransplant } \\
\text { Patients } \\
(\mathbf{n}=55)\end{array}$ \\
\hline & $\mathrm{n}(\%)$ & n $(\%)$ & $\mathrm{n}(\%)$ \\
\hline \multicolumn{4}{|l|}{ Normal Scores (1-1.4) } \\
\hline Evoked Score & $21(95 \%)$ & $70(54 \%)$ & $25(46 \%)$ \\
\hline Time-Domain Score & $20(91 \%)$ & $50(38 \%)$ & $26(47 \%)$ \\
\hline Frequency-Domain Score & $19(86 \%)$ & $40(31 \%)$ & $23(42 \%)$ \\
\hline 24-hr HRV Score & $19(86 \%)$ & $41(32 \%)$ & $23(42 \%)$ \\
\hline ASC Score & $21(95 \%)$ & $46(35 \%)$ & $20(36 \%)$ \\
\hline \multicolumn{4}{|l|}{ Borderline Scores (1.5-2.4) } \\
\hline Evoked Score & $1(5 \%)$ & $29(22 \%)$ & $16(29 \%)$ \\
\hline Time-Domain Score & $1(9 \%)$ & $41(32 \%)$ & $13(24 \%)$ \\
\hline Frequency-Domain Score & $3(14 \%)$ & $40(31 \%)$ & $9(16 \%)$ \\
\hline 24-hr HRV Score & $3(14 \%)$ & $34(26 \%)$ & $9(16 \%)$ \\
\hline ASC Score & 0 & $8(6 \%)$ & $3(6 \%)$ \\
\hline \multicolumn{4}{|l|}{ Abnormal Scores $(2.5-3.0)$} \\
\hline Evoked Score & 0 & $31(24 \%)$ & $14(25 \%)$ \\
\hline Time-Domain Score & 0 & $39(30 \%)$ & $16(29 \%)$ \\
\hline Frequency-Domain Score & 0 & $50(38 \%)$ & $23(42 \%)$ \\
\hline 24-hr HRV Score & 0 & $55(42 \%)$ & $23(42 \%)$ \\
\hline ASC Score & $1(5 \%)$ & $76(59 \%)$ & $32(58 \%)$ \\
\hline
\end{tabular}


sensitive. In addition, the frequency measures were more capable of discriminating between parasympathetic and sympathetic modulation 53 and documented earlier changes in autonomic function as compared to the time-domain measures. Thus, the frequencydomain measures may be a more clinically useful tool when examining parasympathetic and sympathetic modulation and circadian rhythmicity in longitudinal research protocols.

Essentially no change was seen between pre and posttransplant patients on all study scores. Because previous studies have shown improvement in autonomic function posttransplant ${ }^{99}$, study scores were expected to reflect this change. When examined closely, the posttransplant group was similar to the pretransplant group in factors that have been shown to affect autonomic function (age and gender). However, the posttransplant group consisted of patients that were 6 to 24 months ( 6 mo., $n=25 ; 12$ mo., $\mathrm{n}=24 ; 24$ mo., $\mathrm{n}=6$ ) posttransplant. Possibly, the restoration of autonomic function occurs more slowly and, thus, a longer time-period than 6-24 months posttransplant is needed to document change. Another potential factor was the number of patients in the posttransplant sample (38\%) who had diabetes mellitus pretransplant as compared to the number of patients in the pretransplant sample (23\%) who have diabetes. Although the posttransplant patients no longer have diabetes, their pretransplant pathology may have been such that the posttransplant sample were a select group with poorer function $53,87,93$. It may take more time than $6-24$ months or larger samples with patients who are 12-24 months posttransplant to measure improvement in autonomic function. Future studies examining long-term posttransplant samples need to take into 
consideration such factors as length of pretransplant diabetes and length of dialysis when interpreting study results.

Studies 2, 46 have shown high correlations between some time- and frequencydomain measures. It has been suggested that these measures can be used as surrogates for each other when compared to a third measure 82 . Our study findings did not support this conclusion. While strong intracorrelations were demonstrated among frequencydomain measures and among time-domain measures, the r-values between measures in the two domains were not as strong. In conjunction with the finding that frequencydomain measures were more sensitive than time-domain measures, the correlations suggested that time- and frequency-domain measures cannot be used as surrogates for each other. However, the strong r-values suggest that the time- and frequency-domain measures can be used to support each other.

Our study's results also demonstrated a strong correlation between time- and frequency-domain group scores $(\mathrm{r}=0.88)$, however, both groups of measures are of clinical utility and it is not suggested that one be used in lieu of the other. The timedomain measures, particularly the SDNN, have been shown to be associated with increased mortality in the non-transplant population 37 and, in a preliminary study, in the transplant population 93 . Although frequency-domain measures were more sensitive than time-domain measures, time-domain measures remain an important tool, and, concurring with prior studies, particularly with patients with established disease.

All symptom and impact scores of the ASC were found to be positively correlated, however, the ASC score was only weakly correlated with the evoked, timeand frequency-domain, and 24-hr HRV scores. Possibly the score correlations were weak 
because the ASC, while able to differentiate between normal and abnormal symptomatology, was less sensitive to midrange symptoms. This supposition is supported by the high percentage of abnormal ASC scores in pre and posttransplant patients (see Table 31). The percentage of abnormal ASC scores implied that the ASC was a sensitive indicator of dysautonomia and its clinical utility in monitoring symptomatology in longitudinal studies was further supported.

Another reason for weak correlations was that the ASC was designed to evaluate autonomic symptoms over numerous body systems, such as cardiovascular, gastrointestinal, urogenital. In contrast, the evoked and 24-hr HRV measures assessed only the cardiovascular system. Because the instruments assessed different systems, correlations were weak. Future studies correlating the ASC with evoked tests that assess other body systems are recommended.

In conclusion, our findings showed 24-hr HRV monitoring to be the most sensitive indicator of autonomic dysfunction. We also found that while the time-domain scores were sensitive indicators of dysautonomia, the frequency-domain scores were more sensitive 87,93 and, thus, may be more clinically useful. This is especially the case when examining parasympathetic and sympathetic modulation and circadian rhythmicity in longitudinal research protocols. However, the time-domain measures have been shown to be clinically useful in identifying an at-risk group for sudden death 37,93 , and, consequently are also important clinical measures. All category symptom and impact scores of the ASC were found to be positively correlated. In addition, the ASC was found to be a sensitive indicator of dysautonomia, thus, its clinical utility in monitoring symptomatology in longitudinal studies was further supported. 


\section{Chapter 5. Summary, Recommendations, and Conclusions}

This series of studies was undertaken to psychometrically assess three tests of autonomic function, two objective tests and one subjective test, and to explore relationships among measures of autonomic function in patients with ESRD and healthy adults. The studies began with the development of an instrument, the Autonomic Symptom Checklist, designed to elicit subjective symptomatology of dysautonomia and concluded with the examination of the sensitivity and clinical utility of three autonomic function tests.

Patients with ESRD, with or without diabetes mellitus, have been well-

documented to have poor objective values of autonomic function ${ }^{3}$. In addition, objective measures of autonomic function have been demonstrated as able to identify an at-risk group for sudden death in the myocardial infarction population 37,122 and in patients with $\mathrm{ESRD}^{93}$. A self-administered instrument designed to evaluate autonomic symptomatology and to allow correlation of symptoms with objective tests would be a useful research tool. However, studies examining symptomatology are scant, with autonomic symptomatology instruments assessing clinical signs in addition to subjective symptoms $25,115,127,128$. Definitive symptoms are thought to occur late in the disease process 60,61 .

Although standard protocols and referent values for evoked tests, objective measures of autonomic function, have been suggested ${ }^{21}$, no one test or battery of tests 
has been established as the gold standard. Instead, recommendations have been made that autonomic function laboratories should establish batteries of tests and referent values based on their unique population of study 5,21 . Recently, recommendations have also been made for the standardization and interpretation of 24-hr HRV monitoring, another objective measure of autonomic function 53 . However, it is yet to be seen how the recommendations will be applied in clinical research protocols.

Because our autonomic function laboratory was recently established, referent values based on healthy individuals in our geographic area and comparisons of sensitivity and clinical utility among three tests of autonomic function were needed. A series of three descriptive studies using samples composed of patients with ESRD and healthy adults was used for this purpose. Study 1, reported in Chapter 2, documented the development and psychometric testing of the Autonomic Symptom Checklist, a selfadministered instrument for assessment of autonomic symptomatology. Study 2, reported in Chapter 3, established referent values for evoked tests, 24-hr HRV monitoring, and the ASC based on values obtained from healthy individuals. These values were then compared to autonomic function test values determined from patients with ESRD. Study 3, reported in Chapter 4, examined the correlation, sensitivity, and clinical utility among three tests of autonomic function.

\section{The Autonomic Symptom Checklist}

The ASC was developed to provide a subjective measure of autonomic symptomatology. This measure could then be correlated with two objective measures of dysautonomia, the evoked test and 24-hr HRV monitoring. The ASC is a self- 
administered checklist developed using a focus group approach and designed to recognize the perceived presence, distribution and severity of autonomic symptoms. The ASC assesses dysautonomia symptoms in 5 categories with 32 total questions, each using a 7-point Likert scale116. Four additional questions address daily life-style habits that may influence autonomic function 21 . The five categories of symptoms are postural hypotension, sudomotor abnormalities, gastrointestinal problems, impotence, and hypoglycemia unawareness 59,61 . In each category a question to obtain a score reflecting patients' perception of how problematic symptoms are is also asked.

Content validity was established by a group of experts. Construct validity was established by administering it to two groups of ESRD patients with known autonomic dysfunction, diabetic ESRD patients $(\mathrm{n}=85)$ and nondiabetic ESRD patients $(\mathrm{n}=159)$ and to healthy controls $(\mathrm{n}=35)$. The DM group reported the greatest symptomatology, followed by the NonDM group, and with least symptoms, the control group. This pattern was present for all symptom categories. There was significant difference $(\mathrm{p} \leq 0.0001)$ among all groups for three symptom scores (orthostatic hypotension, gastrointestinal problems, and impotence) and two impact scores (gastrointestinal problems and impotence). There was significant difference ( $\mathrm{p} \leq 0.003)$ between healthy controls and both ESRD groups for one symptom score (sudomotor abnormalities) and two impact scores (orthostatic hypotension and sudomotor abnormalities). In addition, findings demonstrated a significant difference $(\mathrm{p} \leq 0.03)$ in symptoms between patients with diabetes who were hypoglycemia aware and hypoglycemia unaware. Test-retest reliability for subscales yielded moderate to high r-values ( 0.59 to 1.00$)$, except for the sudomotor subscale. The ASC appropriately differentiated among control and two 
groups with known autonomic dysfunction. In addition, patient groups with diabetes mellitus, who have been shown to have the poorest objective measures of autonomic function 87 , also had the highest symptom scores. Thus, increased symptomatology is seen in patients with worsening physiologic autonomic function.

In addition, the symptomatology of posttransplant patients $(\mathrm{n}=77)$ as compared to pretransplant patients $(\mathrm{n}=233)$ was examined. It was thought that with restoration of

metabolic balance and euglycemia posttransplant 99 , symptomatology would improve. Findings showed the posttransplant sample had statistically significant $(\mathrm{p} \leq 0.05)$ fewer symptoms than the pretransplant sample. This finding further supported the construct validity of the ASC. Thus, the results of these studies indicated the ASC is a potentially useful research instrument for monitoring symptom changes brought about by interventions designed to improve autonomic function.

\section{Referent Values for Evoked Tests, 24-hr HRV Monitoring, and the ASC}

This study followed previously made recommendations 5,21 to establish laboratory and population dependent referent values for measures of autonomic function. The evoked measures (HRDB and VR), the 24-hr HRV time- and frequency-domain measures (total power, HF, LF, SDNN, SDANN, SD, PNN50, and RMSSD), and the ASC index of a convenience sample of pre-kidney or kidney pancreas transplant patients $(\mathrm{n}=363)$ and healthy adults $(\mathrm{n}=158)$ were examined. Characteristic values for healthy individuals and pretransplant patients, in addition to normal, borderline, and abnormal values were determined for each of the study measures. The characteristic values of the two pretransplant ESRD samples (ESRD with DM, n=91; ESRD NonDM, n=272) were 
found to be much poorer, especially those of the ESRD with diabetes group, than those for the healthy individual sample. The abnormal values were found to be effective cutpoints for identifying a group with compromised autonomic function in the two patient samples. In addition, individual frequency-domain measures were more sensitive than either the evoked or time-domain measures. The correlation of age with autonomic function and the effect of gender were examined and found less significant in the patient samples and to vary in importance depending on the measure being studied. This was thought to be due to the disease process attenuating the effects of age and gender on autonomic function. The disease process had a minimal effect on correlations among time- and frequency-domain variables.

\section{Correlation and Sensitivity of Measures of Autonomic Function}

Data from a convenience sample of pre-kidney or kidney-pancreas transplant and posttransplant patients $(n=185)$ and healthy adults $(n=22)$ were evaluated to address correlation and sensitivity of autonomic function tests. Our findings were congruent with others 48,49 , which showed 24-hr HRV monitoring to be the most sensitive indicator of autonomic dysfunction. In addition, while the time-domain scores were sensitive indicators of dysautonomia, the frequency-domain scores were found to be more sensitive, which is also congruent with other studies 87,93 . Because the frequency measures are more capable of discriminating between parasympathetic and sympathetic modulation ${ }^{9}$ as compared to the time-domain measures, the frequency-domain measures may be more useful clinically. Especially when examining parasympathetic and sympathetic modulation and circadian rhythmicity in longitudinal research protocols. 
However, the time-domain measures have been shown to be clinically useful in identifying an at-risk group for sudden death 37,93 , supporting their utility when examining mortality. All category symptom and impact scores of the ASC were found to be positively correlated. In addition, the ASC was found to be a sensitive indicator of dysautonomia.

The onset of dysautonomia at a sub-clinical level at the time of diagnosis 35 has been documented for some patients with diabetes. It is clinically important, therefore, that clinicians have access to sensitive, reliable, noninvasive, and quantitative means of identifying patients at high risk of developing dysautonomia. This early treatment using modalities such as exercise and relaxation 113 to improve autonomic function can be started.

\section{Recommendations}

Three areas of further exploration concerning measurement of autonomic function were identified. These were divided into methodological, theoretical, and clinical recommendations.

\section{Methodological Recommendations}

1. Referent values by gender and by age group need to be established.

2. Recruitment of African-Americans for the healthy adult sample is needed to determine if there are differences in autonomic function by racial status.

3. Focus groups of patients with ESRD are needed to:

- identify questions in the ASC that need clarification. 
- identify and discuss "early" symptoms of dysautonomia as opposed to "late" symptoms, which are already define.

4. Because sudomotor symptom and impact scores were low, in addition to weak correlation coefficients, this category needs to be reexamined for its applicability in the ESRD population.

5. The high reliability coefficients found in the patients with ESRD subgroup need to be confirmed with a larger sample size.

6. Categories of the ASC need to be correlated with other evoked tests that were not examined in this series of studies. For example, the orthostatic hypotension category needs to be correlated with the evoked tilt test measure.

7. The potential clinical utility of other frequencies (ultra low, and very low) needs to be considered by examining their measurement in samples of ESRD transplant patients.

8. To determine whether pretransplant patients with ESRD are a select group of ESRD patients, studies examining autonomic function in ESRD samples not awaiting transplant are needed.

9. Studies are needed that examine long-term transplant patients to evaluate changes in autonomic function from pretransplant.

\section{Theoretical Recommendations}

1. Clinical usefulness of the ASC was shown in this series of studies. However, relationships between the ASC and objective measures needs examining to identify if a score on objective test measures could be strongly correlated with 
symptoms. This could lead to identification of a threshold where symptoms occur.

2. We need to further examine why our referent values are different than those reported by other autonomic function laboratories, particularly in reference to the frequency-domain measures. It has been suggested that this is due to variations in what Hertz ranges are reported by total power, LF, and HF, however, other factors that could be involved are analysis procedure and geographic location.

3. Frequency-domain measures from short-term recordings of HRV (5-15 minutes) are closely correlated to 24-hr HRV frequency measures ${ }^{54}$, and excellent predictors of mortality. If so, short-term recordings could be feasible for longitudinal studies in the ESRD population and needs further exploration.

4. Further studies are needed to confirm that frequency-domain measures are the most sensitive assessment tool for measuring autonomic function.

5. Studies are needed to explore the relationships between interventions, such as exercise and stress reduction, and autonomic function.

\section{Clinical Recommendations}

1. The ASC measured autonomic symptomatology of ESRD patients and its use should be continued. In addition, its ability to monitor variations in symptoms that accompany changes in autonomic function (whether deterioration or improvement) was evident in a small sample of pre and posttransplant patients and needs further study in larger longitudinal studies. 
2. The identified normal, borderline, and abnormal values need to be used in the clinical setting to further establish their clinical utility.

3. To establish clinical utility, the associations among measures of autonomic function and sudden death, particularly the SDNN and frequency-domain measures, need further examination in the ESRD population.

4. The effects of medications, particularly antihypertensive and antidepressants, on autonomic function as measured by $24-\mathrm{hr}$ HRV need further exploration.

5. Longitudinal follow-up studies are needed to examine the effect of interventions, such as transplantation and exercise, on autonomic function.

\section{Conclusions}

This series of studies established the reliability and validity of the ASC, an instrument designed to assess and monitor autonomic symptomatology. Referent values for evoked tests, 24-hr HRV monitoring, and the ASC were established with borderline and abnormal values identified. A scoring system was devised for the three sets of autonomic function tests (evoked, 24-hr HRV, and ASC). It was shown that 24-hr HRV measures, particularly the frequency-domain measures, were more sensitive to dysautonomia as compared to evoked measures. 
List of References 
1. Friedman EA. Management choices in diabetic end-stage renal disease. Nephrology, Dialysis, Transplantation. 1995;10:61-69.

2. Bigger JT, Jr., Fleiss JL, Steinman RC, Rolnitzky LM, Schneider WJ, Stein PK. RR variability in healthy, middle-aged persons compared with patients with chronic coronary heart disease or recent acute myocardial infarction. Circulation. 1995;91:1936-43.

3. Ewing DJ, Martyn CN, Young RJ, Clarke BF. The value of cardiovascular autonomic function tests: 10 years experience in diabetes. Diabetes Care. 1985;8:491-8.

4. Consensus statement: Report and recommendations of the San Antonio conference on diabetic neuropathy. American Diabetes Association and American Academy of Neurology. Diabetes Care. 1988;11:592-7.

5. Low P, Pfeifer MA. Standardization of clinical tests for practice and clinical trials. In: Low PA, ed. Clinical Autonomic Disorders. Boston: Little, Brown and Company; 1993:287-296.

6. Guyton AC, Hall JE. Textbook of Medical Physiology. . 9th ed. Philadelphia: Saunders; 1996.

7. Barron KD, Chokroverty S. Anatomy of the autonomic nervous system: Brain and brainstem. In: Low PA, ed. Clinical Autonomic Disorders. Boston: Little, Brown and Company; 1993:3-15.

8. Harati Y. Anatomy of the spinal and peripheral autonomic nervous system. In: Low PA, ed. Clinical Autonomic Disorders. Boston: Little, Brown and Company; 1993:17-38.

9. Akselrod S, Gordon D, Ubel FA, Shannon DC, Berger AC, Cohen RJ. Power spectrum analysis of heart rate fluctuation: A quantitative probe of beat-to-beat cardiovascular control. Science. 1981;213:220-2.

10. Pomeranz B, Macaulay RJ, Caudill MA, Kutz I, Adam D, Gordon D, Kilborn KM, Barger AC, Shannon DC, Cohen RJ, et al. Assessment of autonomic function in humans by heart rate spectral analysis. Am J Physiol. 1985;248:H1513.

11. Billman GE, Dujardin JP. Dynamic changes in cardiac vagal tone as measured by time-series analysis. Am J Physiol. 1990;258:H896-902.

12. Campese VM, Romoff MS, Levitan D, Lane K, Massry SG. Mechanisms of autonomic nervous system dysfunction in uremia. Kidney International. 1981;20:246-253.

13. Campese VM, Massry SG. Autonomic Nervous System. In: Massry SG, Glassock RJ, eds. Textbook of Nephrology. Baltimore: Williams \& Wilkins; 1989:11621165 .

14. Greene DA, Feldman EL, Stevens MJ, Sima AAF, Albers JW, Pfeifer MA. Diabetic neuropathy. In: Porte D, Jr., Sherwin RS, eds. Ellenberg and Rifkin's diabetes mellitus: Theory and practice. 5th ed. Stamford, Connecticut: Appleton \& Lange; 1997:1423.

15. Sharpey-Schafer E. Effect of valsalva's manoeuvre on the normal and failing circulation. British Medical Journal. 1955;1:693-695. 
16. Katz LA, Spiro HM. Gastrointestinal manifestations of diabetes. N Engl J Med. 1966;275:1350-61.

17. Ewing DJ. Cardiovascular reflexes and autonomic neuropathy. Clin Sci Mol Med. 1978;55:321-7.

18. Clarke BF, Ewing DJ, Campbell IW. Diabetic autonomic neuropathy. Diabetologia. 1979;17:195-212.

19. Ewing DJ, Campbell IW, Burt AA, Clarke BF. Vascular reflexes in diabetic autonomic neuropathy. Lancet. 1973;2:1354-6.

20. Ewing DJ, Winney R. Autonomic function in patients with chronic renal failure on intermittent haemodialysis. Nephron. 1975;15:424-9.

21. Proceedings of a consensus development conference on standardized measures in diabetic neuropathy. Summary and recommendations. Diabetes Care. 1992; 15:1104-7.

22. Dyrberg T, Benn J, Christiansen JS, Hilsted J, Nerup J. Prevalence of diabetic autonomic neuropathy measured by simple bedside tests. Diabetologia. 1981;20:190-4.

23. Hartwig MS, Cardoso SS, Hathaway DK, Gaber AO. Reliability and validity of cardiovascular and vasomotor autonomic function tests [see comments]. Diabetes Care. 1994; 17:1433-40.

24. Hathaway DK, Abell T, Cardoso S, Hartwig MS, El-Gebely S, Gaber AO. Improvement in autonomic and gastric function following pancreas-kidney versus kidney-alone transplantation and the correlation with quality of life. Transplantation. 1994;57:816-22.

25. Rothschild AH, Weinberg CR, Halter JB, Porte D, Pfeifer MA. Sensitivity of R-R variation and Valsalva ratio in assessment of cardiovascular diabetic autonomic neuropathy. Diabetes Care. 1987;10:735-41.

26. Braune S, Schulte-Monting J, Schwerbrock S, Lucking CH. Retest variation of cardiovascular parameters in autonomic testing. J Auton Nerv Syst. 1996;60:1037.

27. Braune S, Auer A, Schulte-Monting J, Schwerbrock S, Lucking CH. Cardiovascular parameters: Sensitivity to detect autonomic dysfunction and influence of age and sex in normal subjects. Clin Auton Res. 1996;6:3-15.

28. Chu TS, Tsai TJ, Lai JS, Chen WY. Evaluation of cardiovascular autonomic function tests in normal subjects. Taiwan I Hsueh Hui Tsa Chih. 1989;88:404-6.

29. Chu TS, Tsai TJ, Lee SH, Yen TS. Evaluation of cardiovascular autonomic function tests in dialysis patients. J Formos Med Assoc. 1993;92:237-40.

30. Tsai TJ, Chen YM, Chu TS, Wu CF, Tai TY. Cardiovascular autonomic dysfunction in non-insulin-dependent diabetes mellitus and its relation to renal dysfunction. J Formos Med Assoc. 1993;92:509-13.

31. Low PA, Denq JC, Opfer-Gehrking TL, Dyck PJ, O' Brien PC, Slezak JM. Effect of age and gender on sudomotor and cardiovagal function and blood pressure response to tilt in normal subjects. Muscle Nerve. 1997;20:1561-8.

32. Opfer-Gehrking TL, Low PA. Impaired respiratory sinus arrhythmia with paradoxically normal Valsalva ratio indicates combined cardiovagal and peripheral adrenergic failure. Clin Auton Res. 1993;3:169-73. 
33. Ewing DJ. Noninvasive evaluation of heart rate: The time domain. In: Low PA, ed. Clinical Autonomic Disorders: Evaluation and Management. Boston: Little, Brown and Company; 1993:297-314.

34. Hathaway DK, Tolley EA, Blakely ML, Winsett RP, Gaber AO. Development of an index to predict posttransplant diabetes mellitus. Clinical Transplantation. 1993;7:330-338.

35. Ziegler D, Dannehl K, Mühlen H, Spüler M, Volksw D, Gries FA. Prevalence of cardiovascular autonomic dysfunction assessed by spectral analysis and standard tests of heart-rate variation in newly diagnosed IDDM patients. Diabetes Care. 1992;15:908-11.

36. Billman GE, Schwartz PJ, Stone HL. The effects of daily exercise on susceptibility to sudden cardiac death. Circulation. 1984;69:1182-9.

37. Kleiger RE, Miller JP, Bigger JT, Jr., Moss AJ, the Multicenter Postinfarction Research Group. Decreased heart rate variability and its association with increased mortality after acute myocardial infarction. Am J Cardiol. 1987;59:25662.

38. Bigger JT, Jr., Fleiss JL, Rolnitzky LM, Steinman RC. Frequency domain measures of heart period variability to assess risk late after myocardial infarction. J Am Coll Cardiol. 1993;21:729-36.

39. Cowan MJ, Pike K, Burr RL, Cain KC, Narayanan SB. Description of time- and frequency- domain-based measures of heart rate variability in individuals taking antiarrhythmics, beta blockers, calcium channel blockers, and/or antihypertensive drugs after sudden cardiac arrest. J Electrocardiol. 1993;26 Suppl:1-13.

40. Hathaway DK, El-Gebely S, Cardoso SS, Elmer DS, Gaber AO. Autonomic cardiac dysfunction in diabetic transplant recipients succumbing to sudden cardiac death. Transplantation. 1995;59:634-7.

41. Navarro X, Kennedy WR, Aeppli D, Sutherland DE. Neuropathy and mortality in diabetes: Influence of pancreas transplantation. Muscle Nerve. 1996;19:1009-16.

42. Bernardi L, Ricordi L, Lazzari P, Solda P, Calciati A, Ferrari MR, Vandea I, Finardi G, Fratino P. Impaired circadian modulation of sympathovagal activity in diabetes. A possible explanation for altered temporal onset of cardiovascular disease. Circulation. 1992;86:1443-52.

43. Spallone V, Bernardi L, Ricordi L, Soldà P, Maiello MR, Calciati A, Gambardella S, Fratino P, Menzinger G. Relationship between the circadian rhythms of blood pressure and sympathovagal balance in diabetic autonomic neuropathy. Diabetes. 1993;42:1745-52.

44. Hjalmarson A, Gilpin EA, Nicod P, Dittrich H, Henning H, Engler R, Blacky AR, Smith SC, Jr., Ricou F, Ross J, Jr. Differing circadian patterns of symptom onset in subgroups of patients with acute myocardial infarction. Circulation. 1989;80:267-75.

45. Freeman R, Saul JP, Roberts MS, Berger RD, Broadbridge C, Cohen RJ. Spectral analysis of heart rate in diabetic autonomic neuropathy. A comparison with standard tests of autonomic function. Arch Neurol. 1991;48:185-90.

46. Kleiger RE, Bigger JT, Bosner MS, Chung MK, Cook JR, Rolnitzky LM, Steinman R, Fleiss JL. Stability over time of variables measuring heart rate variability in normal subjects. Am J Cardiol. 1991;68:626-30. 
47. van Ravenswaaij-Arts CMA, Kollee LAA, Hopman JCW, Stoelinga GBA, van Geijn HP. Heart rate variability. Ann Intern Med. 1993;118:436-47.

48. Ewing DJ, Neilson JM, Travis P. New method for assessing cardiac parasympathetic activity using 24 hour electrocardiograms. Br Heart J. 1984;52:396-402.

49. Comi G, Sora MG, Bianchi A, Bontempi B, Gianoglio P, Cerutti S, Micossi P, Canal N. Spectral analysis of short-term heart rate variability in diabetic patients. J Auton Nerv Syst. 1990;30 Suppl:S45-9.

50. Lishner M, Akselrod S, Avi VM, Oz O, Divon M, Ravid M. Spectral analysis of heart rate fluctuations. A non-invasive, sensitive method for the early diagnosis of autonomic neuropathy in diabetes mellitus. J Auton Nerv Syst. 1987;19:119-25.

51. Thomaseth K, Cobelli C, Bellavere F, Balzani I, De Masi G, Bax G, Carenza P. Heart rate spectral analysis for assessing autonomic regulation in diabetic patients. J Auton Nerv Syst. 1990;30 Suppl:S169-71.

52. Cloarec-Blanchard L, Girard A, Houhou S, Grünfeld J-P, Elghozi J-L. Spectral analysis of short-term blood pressure and heart rate variability in uremic patients. Kidney Int Suppl. 1992;37:S14-8.

53. Heart rate variability: standards of measurement, physiological interpretation and clinical use. Task Force of the European Society of Cardiology and the North American Society of Pacing and Electrophysiology [see comments]. Circulation. 1996;93:1043-65.

54. Bigger JT, Fleiss JL, Rolnitzky LM, Steinman RC. The ability of several shortterm measures of RR variability to predict mortality after myocardial infarction. Circulation. 1993;88:927-34.

55. Kautzner J. Reproducibility of heart rate variability measurement. In: Malik M, Camm AJ, eds. Heart rate variability. Armonk, New York: Futura; 1995:165171.

56. Booudreaux-Bartels GF, Murray R. Time-Frequency Signal Representations for Biomedical Signals. In: Bronzino JD, ed. The Biomedical Engineering Handbook. Boca Raton, Florida: CRC Press, Inc.; 1995:2862.

57. Kleiger RE, Stein PK, Bosner MS, Rottman JN. Time-domain measurements of heart rate variability. In: Malik M, Camm AJ, eds. Heart Rate Variability. Armonk, NY: Futura Publishing Company; 1995:33-45.

58. Camm AJ, Fei L. Risk Stratification Following Myocardial Infarction: Heart Rate Variability and Other Risk Factors. In: Malik M, Camm AJ, eds. Heart Rate Variability. Armonk, NY: Futura Publishing; 1995:369-392.

59. Hosking DJ, Bennett T, Hampton JR. Diabetic autonomic neuropathy. Diabetes. 1978;27:1043-55.

60. Rundles RW. Diabetic neuropathy. Medicine (Baltimore). 1945;24:111-160.

61. Ewing DJ, Campbell IW, Clarke BF. The natural history of diabetic autonomic neuropathy. $Q$ J Med. 1980;49:95-108.

62. Winsett RP. IGG autoantibodies and diabetic gastroenteropathies. . Graduate Health Sciences. Memphis: Doctoral Dissertation, University of Tennessee, Memphis; 1997:1-145. 
63. Mackay JD, Page MM, Cambridge J, Watkins PJ. Diabetic autonomic neuropathy. The diagnostic value of heart rate monitoring. Diabetologia. 1980;18:471-8.

64. De Meersman RE. Heart rate variability and aerobic fitness. Am Heart J. 1993;125:726-31.

65. Cowan MJ, Pike K, Burr RL. Effects of Gender and Age on Heart Rate Variability in Healthy Individuals and in Persons After Sudden Cardiac Arrest. Journal of Electrocardiology. 1994;27, Supplement:1-9.

66. Goldsmith RL, Bigger JT, Jr., Steinman RC, Fleiss JL. Comparison of 24-hour parasympathetic activity in endurance-trained and untrained young men. $J \mathrm{Am}$ Coll Cardiol. 1992;20:552-8.

67. Fogel RI, Balady GJ, O'Malley C, Williams P, Ryan TJ. Exercise conditioning in cardiac patients does not effect HRV despite attainment of a training effect. Circulation. 1992;86:I-589.

68. Levy WC, Cerqueira MD, Harp GD, Abrass IB, Schwartz RS, Stratton JR. Exercise training increases heart rate variability in healthy young and elderly males. Circulation. 1992;86:I-588.

69. Singer DH, Ori Z. Changes in Heart Rate Variability Associated with Sudden Cardiac Death. In: Malik M, Camm AJ, eds. Heart Rate Variability. Armonk, NY: Futura; 1995:429-448.

70. Brembilla-Perrot B, Jacquemin L, Beurrier D. Relationships between heart rate variability and antiarrhythmic effects of hydroquinidine. Cardiovasc Drugs Ther. 1997;11:493-8.

71. Brembilla-Perrot B, Alsagheer S, Jacquemin L, Beurrier D, Retournay G, Grentzinger A. [Influence of anti-arrhythmia agents on heart rate variability]. Ann Cardiol Angeiol (Paris). 1997;46:129-34.

72. Hathaway D. personal communication. ; 1998.

73. Low P. Laboratory evaluation of autonomic failure. In: Low PA, ed. Clinical Autonomic Disorders. Boston: Little, Brown and Company; 1993:169-195.

74. Wheeler T, Watkins PJ. Cardiac denervation in diabetes. Br Med J. 1973;4:584-6.

75. Cowan MJ. Measurement of heart rate variability. West J Nurs Res. 1995;17:3248; discussion 101-11.

76. Hathaway D, Gaber AO. Autonomic function and posttransplant quality of life. . Memphis, TN: NIH Grant No. RO1-NR03871, University of Tennessee, Memphis; 1995.

77. Bigger JT, Jr., Fleiss JL, Rolnitzky LM, Steinman RC, Schneider WJ. Time course of recovery of heart period variability after myocardial infarction. J Am Coll Cardiol. 1991;18:1643-9.

78. Baselli G, Cerutti S, Civardi S, Lombardi F, Malliani A, Merri M, Pagani M, Rizzo G. Heart rate variability signal processing: a quantitative approach as an aid to diagnosis in cardiovascular pathologies. Int J Biomed Comput. 1987;20:51-70.

79. Baselli G, Porta A, Ferrari G. Models for the analysis of cardiovascular variability signals. In: Malik M, Camm AJ, eds. Heart rate variability. Armonk, New York: Futura; 1995:135-145.

80. Burr RL, Cowan MJ. Autoregressive spectral models of heart rate variability. Practical issues. J Electrocardiol. 1992;25 Suppl:224-33. 
81. Cowan MJ, Burr RL, Narayanan SB, Buzaitis A, Strasser M, Busch S.

Comparison of autoregression and fast Fourier transform techniques for power spectral analysis of heart period variability of persons with sudden cardiac arrest before and after therapy to increase heart period variability. J Electrocardiol. 1992;25 Suppl:234-9.

82. Bigger JT, Jr., Fleiss JL, Steinman RC, Rolnitzky LM, Kleiger RE, Rottman JN. Correlations among time and frequency domain measures of heart period variability two weeks after acute myocardial infarction. Am J Cardiol. 1992;69:891-8.

83. Keehn M. Heart Rate Variability Physician's Guide. . 2nd ed. Milwaukee: Marquette Electronics, Inc; 1992.

84. Bigger JT, Jr., Albrecht P, Steinman RC, Rolnitzky LM, Fleiss JL, Cohen RJ. Comparison of time- and frequency domain-based measures of cardiac parasympathetic activity in Holter recordings after myocardial infarction. Am J Cardiol. 1989;64:536-8.

85. Bigger JT, Jr., Steinman RC, Rolnitzky LM, Fleiss JL, Albrecht P, Cohen RJ. Power law behavior of RR-interval variability in healthy middle-aged persons, patients with recent acute myocardial infarction, and patients with heart transplants. Circulation. 1996;93:2142-51.

86. Kirvela M, Salmela K, Toivonen L, Koivusalo AM, Lindgren L. Heart rate variability in diabetic and non-diabetic renal transplant patients. Acta Anaesthesiol Scand. 1996;40:804-8.

87. Hathaway DK, Cashion AK, Wicks MN, Milstead EJ, Gaber AO. Cardiovascular dysautonomia of patients with end-stage renal disease and type I or type II diabetes. Nursing Research. 1998;in press.

88. Strano S, De Castro S, Urani C, Ferrucci A, Marcheselli A, Poli L, Berloco P, Calcagnini G, Jr., Cerutti S, Cortesini R. Power spectrum analysis of heart rate variability following kidney transplantation. Transplant Proc. 1993;25:2600-1.

89. Bigger JT, Jr., Fleiss JL, Rolnitzky LM, Steinman RC. Stability over time of heart period variability in patients with previous myocardial infarction and ventricular arrhythmias. Am J Cardiol. 1992;69:718-739.

90. Hennekens CH, Buring JE. Epidemiology. In: Mayrent SL, ed: Little, Brown and Company; 1987:383.

91. Malik M, Hnatkova K, Camm AJ. Practicality of postinfarction risk assessment based on time-domain measurement of heart rate variability. In: Malik M, Camm AJ, eds. Heart Rate Variability. Armonk, NY: Futura Publishing; 1995:393-405.

92. Singer DH, Martin GJ, Magid N, Weiss JS, Schaad JW, Kehoe R, Zheutlin T, Fintel DJ, Hsieh A-M, Lesch M. Low heart rate variability and sudden cardiac death. J Electrocardiol. 1988;21 Suppl:S46-55.

93. Hathaway DK, Cashion AK, Milstead EJ, Winsett RP, Cowan PA, Wicks MN, Gaber AO. Autonomic dysregulation in patients awaiting kidney transplantation. American Journal of Kidney Disease. 1998;in press.

94. Kennedy HL, Bavishi NS, Buckingham TA. Ambulatory (Holter) electrocardiography signal-averaging: a current perspective. Am Heart J. 1992;124:1339-46. 
95. Mainardi LT, Bianchi AM, Cerutti S. Digital Biomedical Signal Acquisition and Processing. In: Bronzino JD, ed. The Biomedical Engineering Handbook. Boca Raton, Florida: CRC Press, Inc.; 1995:2862.

96. Malik M, Camm. AJ. Components of heart rate variability--what they really mean and what we really measure [editorial] [see comments]. Am J Cardiol. 1993;72:821-2.

97. Sanderson JE. Limitations of heart rate variability measurements [letter; comment]. Am J Cardiol. 1994;74:417.

98. Algra A, Tijssen JG, Roelandt JR, Pool J, Lubsen J. Heart rate variability from 24-hour electrocardiography and the 2-year risk for sudden death. Circulation. 1993;88:180-5.

99. Hathaway D, Abell T, Cardoso S, Hartwig M, Elmer D, Horton J, Lawrence D, Gaber L, Gaber AO. Improvement in autonomic function following pancreaskidney versus kidney-alone transplantation. Transplant Proc. 1993;25:1306-8.

100. Gaber AO, Cardoso S, Pearson S, Abell T, Gaber L, Hathaway D, Alakkad M, Cromer R, Britt LG. Improvement in autonomic function following combined pancreas-kidney transplantation. Transplant Proc. 1991;23:1660-2.

101. Fraser CL, Arieff AI. Nervous system manifestations of renal failure. In: Schrier RW, Gottschalk CW, eds. Diseases of the kidney. 6th ed. Boston: Little, Brown and Company; 1997:2625-2646.

102. Fraser CL. Neuologic manifestations of renal failure. In: Greenberg A, Cheung AK, Falk RJ, Coffman TM, Jennette JC, eds. Primer on kidney diseases. San Diego: Academic Press; 1994:304-309.

103. Weinrauch LA, JA DE, Gleason RE, Keough J, Mann D, Kennedy FP. Autonomic function in type I diabetes mellitus complicated by nephropathy. A cross-sectional analysis in the presymptomatic phase. Am J Hypertens. 1995;8:782-9.

104. Simmons D. Pathogenesis of diabetic neuropathy. In: Kahn CR, Weir GC, eds. Joslin's Diabetes Mellitus. 13th ed. Philadelphia: Lee and Febiger; 1994:665-690.

105. Dyck PJ, Sherman WR, Hallcher LM, Service FJ, PC OB, Grina LA, Palumbo PJ, Swanson CJ. Human diabetic endoneurial sorbitol, fructose, and myo-inositol related to sural nerve morphometry. Ann Neurol. 1980;8:590-6.

106. Low PA, Dyck PJ. Pathologic studies and the nerve biopsy in autonomic neuropathies. In: Low PA, ed. Clinical Autonomic Disorders. Boston: Little, Brown and Company; 1993:331-344.

107. Shigeta H, Yamaguchi M, Nakano K, Obayashi H, Takemura R, Fukui M, Fujii M, Yoshimori K, Hasegawa G, Nakamura N, Kitagawa Y, Kondo M. Serum autoantibodies against sulfatide and phospholipid in NIDDM patients with diabetic neuropathy [In Process Citation]. Diabetes Care. 1997;20:1896-9.

108. The Diabetes Control and Complications Trial Research Group. The effect of intensive treatment of diabetes on the development and progression of long-term complications in insulin-dependent diabetes mellitus. The Diabetes Control and Complications Trial Research Group. N Engl J Med. 1993;329:977-86.

109. Mølgaard H, Christensen PD, Sørensen KE, Christensen CK, Mogensen CE. Association of 24-h cardiac parasympathetic activity and degree of nephropathy in IDDM patients. Diabetes. 1992;41:812-7. 
110. Gaber AO, el-Gebely S, Sugathan P, Elmer DS, Hathaway DK, McCully RB, Shokouh-Amiri MH, Burlew BS. Changes in cardiac function of type I diabetics following pancreas- kidney and kidney-alone transplantation. Transplant Proc. 1995;27:1322-3.

111. Mølgaard H, Sørensen KE, Bjerregaard P. Circadian variation and influence of risk factors on heart rate variability in healthy subjects. Am J Cardiol. 1991;68:777-84.

112. Kennedy WR, Navarro X, Sakuta M, Mandell H, Knox CK, Sutherland DE. Physiological and clinical correlates of cardiorespiratory reflexes in diabetes mellitus. Diabetes Care. 1989;12:399-408.

113. Cowan MJ, Kogan H, Burr R, Hendershot S, Buchanan L. Power spectral analysis of heart rate variability after biofeedback training. J Electrocardiol. 1990;23 Suppl:85-94.

114. Low PA, Opfer-Gehrking TL, Zimmerman IR, PC OB. Evaluation of heart rate changes: electrocardiographic versus photoplethysmographic methods. Clin Auton Res. 1997;7:65-8.

115. Low P. Appendix--symptomotology. In: Low PA, ed. Clinical Autonomic Disorders: Evaluation and Management. Boston: Little, Brown and Company; 1993.

116. Burns N, Grove SK. The practice of nursing research: conduct, critique, and utilization. . 2nd ed. Philadelphia: W.B. Saunders; 1993:1-818.

117. Huck SW, Cormier WH. Reading statistics and research. . 2nd ed. New York: HarperCollins; 1996:1-652.

118. ADA-AAN. Consensus Statement: Diabetic Neuropathy. Diabetes Care. 1990;13:47-52.

119. Hilsted J, Jensen SB. A simple test for autonomic neuropathy in juvenile diabetics. Acta Med Scand. 1979;205:385-7.

120. Sundkvist G, Almer L, Lilja B. Respiratory influence on heart rate in diabetes mellitus. Br Med J. 1979;1:924-5.

121. Myers GA, Martin GJ, Magid NM, Barnett PS, Schaad JW, Weiss JS, Lesch M, Singer DH. Power spectral analysis of heart rate variability in sudden cardiac death: comparison to other methods. IEEE Trans Biomed Eng. 1986;33:1149-56.

122. Bigger JT, Jr., Fleiss JL, Steinman RC, Rolnitzky LM, Kleiger RE, Rottman JN. Frequency domain measures of heart period variability and mortality after myocardial infarction. Circulation. 1992;85:164-71.

123. Navarro X, Kennedy WR, Loewenson RB, Sutherland DE. Influence of pancreas transplantation on cardiorespiratory reflexes, nerve conduction, and mortality in diabetes mellitus. Diabetes. 1990;39:802-6.

124. Akselrod S, Gordon D, Madwed JB, Snidman NC, Shannon DC, Cohen RJ. Hemodynamic regulation: investigation by spectral analysis. Am J Physiol. 1985;249:H867-75.

125. Assessment: Clinical autonomic testing report of the Therapeutics and Technology Assessment Subcommittee of the American Academy of Neurology. Neurology. 1996;46:873-80.

126. Lown B, Verrier RL. Neural activity and ventricular fibrillation. $N$ Engl J Med. 1976;294:1165-70. 
127. Low PA. Composite autonomic scoring scale for laboratory quantification of generalized autonomic failure. Mayo Clin Proc. 1993;68:748-52.

128. Dyck PJ, Bushek W, Spring EM, Karnes JL, Litchy WJ, PC OB, Service FJ. Vibratory and cooling detection thresholds compared with other tests in diagnosing and staging diabetic neuropathy. Diabetes Care. 1987;10:432-40. 
Appendices 


\section{Appendix A}

\section{Testing and Analysis Procedures for Evoked and 24-hr HRV Monitoring}

PROTOCOL \#: 02

PAGE 1 OF $\underline{2}$

\section{PRE-TEST PREPARATION}

1. Prepare computer for documentation:

A. Open Mac HardDrive

B. Open Folder: BioPac

C. Open Test \#1

D. At the top of screen, click on MP 100. Scroll down to: "Show Input Values". A screen will appear in the upper right corner with scores to be used to input patient values during testing. Use this screen to note room temperature, blood pressures, and finger temperature before and after the ice test.

2. At the start of each test, a dialogue box will appear asking if operator desires to overwrite existing data. Click OK.

3. Tape temperature probe to the upper right corner of the computer table. Do not allow the temperature transducer surface (shiny side) touch any object. This probe will monitor the room temperature until its use with patient monitoring.

4. Insure that the room temperature is between $25^{\circ}-27^{\circ}$ Centigrade using the temperature probe value displayed on the Input Value screen.

5. Complete the patient interview/data form. Place "NA" in areas which are not applicable. Document patient's health history in the right corner of the medication box: i.e. length of IDDM, kidney disease, HTN, dialysis, and any other appropriate information.

6. If patient is a diabetic, insure the Blood Glucose has been drawn prior to starting the tests. Document the results in the Pre-Test BG box on the patient interview/data form.

NOTE: If the Blood Glucose level prior to testing is $>200$ or $<100$, recheck the level after completing the tests and document the results in the Post Test BG box.Continuously monitor diabetic patients during testing. Observe for alertness, profuse diaphoresis, or other signs/symptoms of hypoglycemia. Treat as appropriate or seek immediate assistance. 
7. Gather supplies:

A. Razor and Shaving Cream (if needed)

B. Soap and Water to clean skin (if needed)

C. Alcohol $2 \times 2 \mathrm{~s}$

D. Gauze Pads

E. EKG Electrode Pads (If patient is to receive Holter monitoring following testing, seven (7) electrodes are needed. If Holter monitoring is not to be done, only five (5) electrodes are needed).

F. Transparent/Non-Allergic Tape

G. Double Sided Scotch Tape

H. Scotch Tape

I. Stop Watch

J. $\quad$ Finger Cot

K. Paper Towels

L. Hudson RCl Disposable Mouthpiece

M. $\quad$ Plastic Bowl and ZipLock Bag filled with ice

N. Stool (on which to place bowl of ice water)

O. Holter Monitoring Equipment (See protocol)

8. Ensure the restraining belt is in proper position on the Tilt Table. Cover the table pillows with a clean blue pad.

9. Insure that the following equipment is in place and functional:

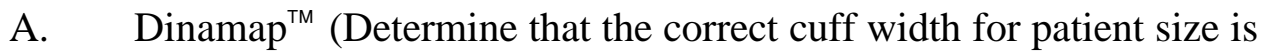
attached to cable. Cuff can be changed by unscrewing the two rubber tubes on the cuff from the equipment cables.)

B. Tilt Table with tilting control box accessible

C. Gastric Leads (white and red) located on hook on the left side of computer table

D. Donor Chair with two (2) armrests

E. EKG leads (white, red, and black) located on hook on the right side of computer table

F. Skin Temperature Probe located on hook on the right side of computer table

G. PPG Signal Probe located on hook on the right side of computer table

H. Valsalva Pressure Monitor Apparatus 


\section{TEST \# FOUR (4): Change in Heart Rate with Deep Breathing (HRDB) and Valsalva Maneuver}

$\underline{\text { Heart Rate Change with Deep Breathing (HRDB) }}$

1. Attach EKG leads to patient:

\begin{tabular}{|c|c|}
\hline \multicolumn{2}{|r|}{ ELECTRODE PLACEMENT } \\
\hline$\angle E A D C O L O R$ & LOCATION \\
\hline White: V1 (mod) & Mid-Sternum-Over Manubrium 1.5 inches from clavicle \\
\hline Black: V4R & Right Mid-Clavicular Line-Below Rib Cage \\
\hline Red: & Left Mid-Clavicular Line-Below Rib Cage \\
\hline
\end{tabular}

2. Patient Instructions:

Explain that they will be doing a breathing exercise which will consist of one minute of continuous breathing in which they will breathe in for five (5) seconds then breathe out for five (5) seconds.

3. Practice for 1-2 breaths. Count for the patient while you are verifying the correct timing with the stop watch: In-2-3-4-5 Out-2-3-4-5.

4. When you are assured that patient understands and can correctly do the test, complete the following tasks simultaneously:

A. Note the starting point on the stop watch which will establish the start of one (1) minute.

B. Begin test by instructing patient to start deep breathing using stop watch to validate time. Collect data for one (1) minute.

C. Create a "carrot" and document in the longitudinal box: "Start deep breathing".

D. Count for patient: In -2-3-4-5 Out-2-3-4-5.

5. At completion of one (1) minute (six in and out breathing cycles), instruct patient to breathe normally.

6. Create a carrot and document in longitudinal box: "Stop deep breathing".

7. Do NOT stop watch. Continue to next test.

\section{VALSALVA MANEUVER (VR)}

1. Preparation:

A. Place the white mouthpiece into the Valsalva apparatus.

B. Position the equipment on the patient's left side where both the patient and technician can visualize the numbers on the meter.

2. Patient Instructions: 
A. Place the Hudson RCI disposable mouth piece in mouth and hold it with one hand.

B. Blow into the mouth piece until the pressure gauge reaches $40 \mathrm{mmHg}$ and maintain that level of blowing for 5-6 seconds. In the actual test instruct patient that they will be maintaining the pressure level for 15 seconds.

C. Upon completion of test, they will be instructed to stop blowing. Continue to rest quietly and breathe normally for an additional 15 seconds.

3. Practice 1-2 times to insure patient's level of understanding as well as ability to reach and maintain the appropriate pressure level. Improper mouth seal, dentures or braces may interfere with quality of test. Practice with rest periods until patient appears able to successfully complete test.

4. Allow patient to rest a minimum of one (1) minute before starting test.

5. Alert patient when you are 10 seconds from starting test and instruct them to place mouthpiece in mouth and wait until they are instructed to "Blow".

6. Complete the following tasks simultaneously:

A. At the start time instruct patient to get a deep breathe and "Blow". Continue saying "Blow" until test is over.

B. Create a "carrot" and document in the longitudinal box: "Start Valsalva".

C. At the point of blowing exhalation, observe watch for fifteen (15) second time frame.

7. At completion of 15 seconds, tell patient to stop blowing. Create a "carrot" and document: "Stop Valsalva".

8. Remind patient to rest quietly/no moving/breathe normally. Maintain quiet for an additional 15 seconds. 


\section{4-hr HRV HOLTER MONITORING: PATIENT PREPARATION/ELECTRODE PLACEMENT}

1.To minimize electrode problems, ensure good quality by checking for expiration date. patient is to only have Holter Monitoring, use 5 electrodes. If Autonomic Functionis also is to be done, use 7 electrodes.

2. Apply shaving lotion and using safety razor, shave individual sites. Shaving improves conductivity, holds electrode to the skin, and facilitates removal of electrode.

3. If skin is extremely oily, first wash designated area with soap and water. Clean site with alcohol swab. Allow to air dry or dry with clean $4 \times 4$ s.

4. Apply "dot" of liquid sandpaper, rubbing into skin with $4 \mathrm{X} 4 \mathrm{~s}$, or use sandpaper squares to further prepare each individual electrode site.

5. If patient is to only receive Holter testing, connect lead wires to each electrode prior to applying to patient. If patient is to have Autonomic Function testing completed first, do not attach leads prior to electrode placement.

6. Apply electrodes (See picture, $\mathrm{p}$ 2). Tape each lead wire to the chest using a stress loop to prevent the wires from pulling directly on the electrode contact points. The loops should be approximately 1 to 2 inches in diameter, about 2 inches from the electrodes (See picture below).

\begin{tabular}{|c|ll|}
\hline \multicolumn{3}{|c|}{ ELECTRODE PLACEMENT } \\
\hline LEAD COLOR & \multicolumn{2}{c|}{ LOCATION } \\
\hline White & Mid-Sternum-Over Manubrium & 1.5 inches from clavicle \\
\hline Green & Right Mid-Clavicular Line & Below Costal Margin \\
\hline Red & Left Mid-Clavicular Line & Below Costal Margin \\
\hline Brown & Right Mid-Axillary Line & Below Costal Margin \\
\hline Black & Left Mid-Axillary Line & Below Costal Margin \\
\hline
\end{tabular}




\section{4-hr HRV HOLTER MONITORING: RECORDER PREPARATION}

1. Use a new cassette tape for each patient. A plastic bar inserted into the tape reels signifies no previous use. Remove the bar from the tape. Using a pencil in the tape reel, wind the tape forward until the blank, white pre-tape is beyond the recording site. This procedure will ensure that data is recorded from the onset of tape recording initiation.

2. Document patient identification information on the cassette label.

Patient's Name

Patient's Social Security Number

Test Date

Time Test was Initiated

Recorder Identification Number: This number is written on the outer metal case.

Patient Category: Pre-Kidney Juvenile Women's Study

3. Obtain a new 9-volt alkaline battery.

4. Remove outer metal case of the recorder. Hold the top of the recorder (the end which protrudes beyond the metal case) in one hand. Slide the metal case off with the other hand (See picture on page 3).

5. Install the battery by inserting it into the battery compartment with the positive $(+)$ and negative (-) terminals aligned according to the diagram inside the compartment. The battery should fit securely in the compartment with no space between the bottom of the battery and the bottom of the compartment.

6. Slide the cassette release to the left (see picture). The recording head assembly moves to the OUT position.

7. Place the tape in the recorder with the full reel on the supply (left) hub. Insert the cassette into the recorder by placing the top edge of the cassette in first under the retaining guides and then carefully easing the cassette down over the supply and take-up hubs and the cassette locator pins.

8. Prepare the patient and connect the lead wires.

9. Activate the recorder by pushing the recording head assembly $\mathbb{I N}$ (flush with the side of the recorder). Visualize the take-up hub or the tape reels turning slowly. Leave the case open while completing the additional documentation to ensure recorder is working properly.

10. Complete the patient documentation on the diary:

Patient Name

Patient Social Security Number

Age Sex

Patient's Medications

Date \& Time test is initiated

Serial No: (This number is the Holter Monitor number

Initials of individual completing test in the space for technician

11. Replace the recorder in the metal case and place inside the canvas purse. Place the Velcro tab across the cable/recorder site to prevent it from becoming dislodged. Do not push the inset button as it will erase tape. 
12. Patient Instructions:

A. Maintain a 24 hour diary of activities. Especially note:

Time went to sleep-including naps

Time awoke

Remembrance of any periods of dreaming while asleep

Exercise

Home Blood Glucose Monitoring Levels $<50$ or $>200$

Time On/Off Dialysis

Stressful Experiences

B. Do not open the canvas purse or attempt to remove the case, even when the test is completed.

C. Wear the canvas purse by the shoulder strap or attached to a belt.

D. When the 24 hours of testing have been completed, carefully remove the tape over the lead wires and unsnap them from the electrodes. Place the leadwires, cable, and canvas purse in the white envelope for mailing or return the equipment to the admissions office on first floor of Bowld Hospital. Do not attempt to open the case. Remove the electrodes and discard.

13. If patient wishes to mail-in equipment, provide them with a white envelope and a Fed Ex mailing label (See example). Identify the initials of the principle investigator for test on the form. Maintain the "Sender's Copy" in the Physiology Function Laboratory. Document the monitor number and test date on the Sender's Copy for future tracking purposes if necessary.

14. Document patient information into Daily Log Book and on Bulletin Board:

Name

Date of Test

Patient's Category: Pre-Kidney Juvenile Women's Study

Holter Number

Time Holter applied

How Holter will be returned: Patient in town or per Federal Express

\begin{tabular}{|c|l|}
\hline \multicolumn{2}{|c|}{ RECORDING EQUIPMENT: INTERNAL VIEW } \\
\hline ITEM & \multicolumn{1}{c|}{ DESCRIPTION } \\
\hline A & Supply Hub \\
\hline B & Cassette Locator Pins \\
\hline C & Take-Up Hub \\
\hline D & Battery Compartment \\
\hline E & $\begin{array}{l}\text { Signal Test Connector for a Holter Signal Test Cable (PN 9401-901) for 5- } \\
\text { leadwires }\end{array}$ \\
\hline F & Capstan \\
\hline G & Recording Head Assembly \\
\hline H & Cassette Retaining Guides \\
\hline I & Cassette Release \\
\hline
\end{tabular}




\title{
PROCEDURE FOR CALCULATING \%VC RESULTS
}

\author{
Valsalva Ratio (VR) \\ Change in RRI with Breathirg (DBPM) \\ Percent Vasoconstriction (\%VC)
}

Open the file with $\%$ VC in the title

\section{Valsalva Ratio (VR)}

The purpose is to detemine the valsalva ratio by calculating the RRI " $m$ in" for 15 seconds during the valsalva manuwer and dividing the reault into the RRJ "min" for $1 S$ seccods after the valaalve minuver.

Cloes all chamnels that may be open except for the ECG channel $(t I)$

To do this: Fold the option key down whilo clieking on the small channel indicators in the upper left hand comer, juit above the operr window.

Review the wrveforms and take note of the carrot markers bind comments. Thene should be a carrot marker just prior to the one labeled "Stop Valsalya" that has been left unlabeled. Labed it "Start Valarelva". this is where yon begin the analyais and recoud all the results.

Hightight at the "Stant Valsalya" marker for 15 seconds (Ulae the SC $\Delta \mathrm{T}=$ to get your time comect)

Under Traneform monn, go to Find pmte. In the dialog box change the following:

Function: intervil

Window:

Minimum $=.3$ seconds

Clictor

Marimum= 1.7 aeconds

A new chamnel appears on the screan. Wo are going to roname and rescale this channel.

To do this:

Renaun:

Click ou the left Yaxis In the dialog box change name to "During Valsalva". Click OK Besenle:

Click on the Right $Y$ exis and set the parameters for the scale to .1. Change Midpoint to .6 or 7 .

Now hidhifint the men chnnmel (During Valsalva) around the smallest portion, which is located somewhere ner the ond. Sitce yon are calculating the Minimum RRI it is not necesseny to highlight the entipe wavefarm. It is ok to do the whole wavefonin, juot remember not to inclede the first two stepe and start in the midtile of the wep not at the begining or end of a step.

Recond RRI "min" on the comment bar at the "Stant Valadva" imarker.

EX: ri min/max $1.2 \%$ (max is celculgited nextl)

Go to "Stop Velgalva" mentker.

Hithblight 15 seconds from the "atop valsalva' marker (Use the SC $\Delta T=$ to get your time correct!).

Under Trancintm menu, go to Find Bute. In the dialog box chinge the following;
Function; interval

Window: 


\section{Minimum= 3 seconds \\ Ciek OK \\ Maximum $=1.7$ soconds}

A now channel appenrs on the screen. We ure going to renamo and reacale this channel.

To do this:

Reinare

Click on the left Yaxis. In the dialog box change name to "After Vellalva". Click OK

Rerefle:

Click on the Right $Y$ axis and set the parameters for the scale to .1. Change Midpoint to 6 or

7.

Now hishlight the nep elonnol (After Valsalva) around the langest portion, which ia Iocalod somewhere near the beriming. Since you are calculating the Maximum RRI it is not necessary to highlight the antire wravelorm. It is ok to do the whole waveform, just remember not to include the firgt two steps and start in the middle of the otop not af the beginning or end of a ctop.

Recond RRI "max" on the comment bar at the "Stout Vilpal ve" manter.

EX: mi minimax $1.2 / 1.96$

Calculate VR by dividing the RRI max after by the RRI min during

EX: $\max 1.95=1.6$

$\min 1.2$

Recond the VR on the "Start Velsalva" coment bar mo well an on the wort shect

The conment bar should then read: if min/max $1.2 / 1.96$, ve=1.6

\section{Change in RRI with Breathing (DBPM)}

Scroll through carrox makers antil you find the "Start deep breathing" marker. It may be unlabeled, but yon will recogaize it by the cycic waveforms present Once you find it, lahed the marker "Start deep brenting".

Hightight one minute beginning at the "start deep breathing".

Under Tranctorm menur go to Find Rate. In the dialog box chapge the following:

Function: interval.

Window:

Minimum= 3 soconds

Crick OK

Maximum $=1.7$ beconds

A now chanded apperss on the scroen. Wo are going to renane and roscale this channel.

To do this:

Remanes

Click on the left Yaxis. In the dialog box change name to "DBPM". Cliek OK

Besento:

Click on the Right $Y$ axis and set the parneten for the scalo to .1. Chimge Midpoint to 6 or 7 .

Now bighlight the now efonnel (DBPM) from the begiming, oxcept leave of the first few ateps of the greph. Start in the middle of the step not at the beginning or end of a step. 
At the top of the window, the second function chamel next to SC $\triangle T$ " = thould be set at "min" and the thind chennel should be set at "max". In those windows are the RRI mimimam and maximum.

Becerd min and max on the comment bar. mi mintmax $=32.717 .6$

DIVlde arch into 60 (to obtain beats'min). Record mi min/max $=.551 .72109 / 83$

DAPM = RRI "min" - RRI "max" after tho divi:ion

Recerd DBPM on comment bar 49 well as the wotsheet. mimin/max=55.72; 109\%3; DBPM= 26

\section{Percent Vasoconstriction (\%VC)}

The amount of vigoconstriction is calculated by using the TPA before ice (alneady calculated in the PAR) and the TPA during ice immartion.

Clowe all chanaels that may be open except for the FPG and Temp To do this: Hold the option key down whilo elichines on the small channol infication in the upper left hand conser, just above the cpen window.

Review the wave form by clicting through the carrot marfere until you find the cone labeled "Hand in

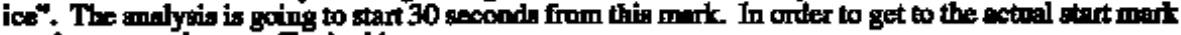
you have to make one. To do this:

Hlgilight 30 seconds from the "Himed in ice" matter (Uhe the SC $\Delta \mathrm{T}=$ to got your time conect!).

Place a new carrot marker here and label "Start beno". This is wherr you begin.

If

SFiteh the first function chamnel to "area". (SC=area)

Pecerd on note ber. EX: area $=15230$ seconds

Mildiply the result by 2.

Bocend the resalt agnin on the note bar. EX: area $=152 / 30$ seconde; 30.4/mimute

Record the one minute result on the workshest for TPA after ice.

Lowk up the TPA before ice from the workbeet to finiet the calenlation.

\%VC = TPA before ice minus TPA after ice + TPA before ice $\times 100$

Bave alce You ane prompted to Roplace. Clict yea Do not nal ame the file. 


\section{HEART RATE VARIABILITY ANALYSIS PROCEDURE}

\section{Loading New Tapes}

A. One tape

1. Select \#1--NEW TAPE from MAIN MENU

2. Select E (Enter) from the NEW TAPE MENU—press RETURN KEY

3. Identify an empty space (1-10), select that number when prompted-press RETURN KEY

4. Respond to prompts from the computer to:

a. Type patient's name-Last name, first name, middle initial (if available)

b. Type of tape-type letter "M"

c. Number of hours to sample-type 24 (hitting return will result in more dual channel noise being identified)

d. Type in Sampling Parameters-Usually chose "T" for template reduction for adults, and "TP" for pediatric patients (do not space or put comma between selections). Additional parameters may be chosen if needed to increase EKG size, read only one channel, etc. Refer to Marquette Manual. Press RETURN KEY.

5. NEW TAPE MENU reappears. Select P (Process) and enter tape number when prompted. Press RETURN KEY.

6. Screen will prompt you to load tape for patient "___ " into Hard Drive Cassette reader. Insert tape into hard drive and press RETURN KEY to have system analyze tape (analysis takes about $20 \mathrm{~min} / \mathrm{tape}$ ). You may change sampling parameter before hitting return key if desired.

7. Place diary and tape in upper tray beside computer monitor.

B. Batch Loading Tapes

1. Select E(Enter) from NEW TAPE MENU

2. Perform steps 1-4 as above for each tape, making sure to select empty space (1-10) for each tape.

3. Select P(Process) from NEW TAPE MENU—Press RETURN KEY when machine asks which tapes to process (this will allow all tapes you "entered" to be processed)

4. Load each tape into the Hard Drive Cassette reader when prompted (takes 3-5 min/tape).

5. Press RETURN KEY after each tape loaded for machine to read tape.

6. Once batch loading is complete, the machine will analyze each tape and screen will be blank.

7. Place diary and tapes into upper tray beside computer monitor.

8. Once MAIN MENU is displayed again, operator may access tapes.

C. Accessing Tapes (if already loaded and analyzed)

1. Select \#1-NEW TAPE MENU from the MAIN MENU

2. Select R(Review) from the NEW TAPE MENU and press RETURN KEY.

3. Enter tape number to be reviewed and press RETURN KEY. > will appear beside selected tape

4. Press ESC to escape to the MAIN MENU and perform functions on the selected tape

II. Patient Information

A. Select $\# 2$ from the MAIN MENU

B. Enter data for all fields using RETURN key or DOWN ARROW to move from one field to another. Data to be entered is on the patient diary. Use military time for time tape started.

C. Press ESC to escape to MAIN MENU.

III. Diary

A. Select \#3 from the MAIN MENU

B. Use "I" to insert time and date of an event. If events are already there, delete them 
C. Once time and date are inserted, select "F" for free-form to write down the "event." Some symptoms can be selected by choosing a letter from the menu at the bottom of the screen

D. Use > KEY to access the next page for diary entries; only 10 entries can be displayed on each page.

E. Verify events and times entered

F. Press ESC to return to MAIN MENU

\section{QRS Morphology Review}
A. Select \#4 from MAIN MENU
B. Review each classification group in the following order: Normal (N), Ventricular (V), Artifact (X), and Unclassified (?)
C. Press TAB KEY, then symbol for classification group you wish to review
D. Individuals who are very active (or if you did not chose template reduction (T) for sampling parameter) tend to have more classes within group.

E. Review the representative beat for each class, use ARROW KEY to move down one class at a time or the > KEY to move to next page of classes

F. Review some beats of each class until comfortable with classification, using "R" KEY and then Up and DOWN ARROW KEYS to see beats in the class. A six second strip which includes the classified beat may be viewed by pressing "S" key. Follow menu on bottom of the screen which prompts you regarding possible choices.

G. Reclassify beats or classes as necessary. Follow screen prompts. Use SPACE BAR to place marker in the strip to relabel a beat. > or < KEYS move cursor to next computer identified beat. RIGHT and LEFT ARROW KEYS allow you to move cursor manually to position cursor exactly on QRS complex.

H. Press ESC KEY to return to MAIN MENU

V. Full Disclosure

A. Select "F" from MAIN MENU

B. Note Dual Channel Noise (DCN) for report

C. If DCN is $>2$ hours attempt to reduce DCN by changing sampling parameters. This requires the tape to reanalyzed by the machine ( 20 minutes). It is recommended that you complete the analysis with the current parameters because a reduction in DCN may not be possible even with the sampling parameters changed.

D. If DCN > 4 hours, even with parameter changes, notify principle investigator to see if holter needs to be repeated.

E. DCN is marked by brackets with channels containing noise $(<3>2)$. DCN is programmed out of the HRV analysis even if you manually correct the beats. We are not relabeling beats in DCN.

F. NOTE: Since DCN is excluded from analysis, episodes of arrhythmias occurring during this time will not be reported. If this is of concern to the investigator, review of DCN can be done by Pressing "D" for DATA WINDOW, "H" for HIGHLIGHT, and then "D" for DUAL CHANNEL NOISE, then press RETURN KEY. Pressing "F" for Forward, will bring the next episode of DCN to the screen for review. The individual strip of DCN can be selected by pressing the "S" obtain the cursor, move the cursor to the area of DCN using the ARROW KEYS and press the "S" for STRIP key again. Follow prompts on the screen.

G. Press ESC KEY to return to MAIN MENU

VI. Episode Review (Histograms)

A. Select \#5 from the MAIN MENU

B. Review R-R intervals, all beats (enter 1, 1) and press RETURN KEY

C. Review shortest RR intervals, correct any mislabeled beats by selecting "S" Strip Once strip appears, press SPACE BAR for cursor to appear, use > or $<$ to move to next sensed 
beat or ARROW KEYS to manually position cursor over QRS to relabel a beat

D. You can view the next RR interval by pressing the UP ARROW KEY, the screen will show the histogram at the top of the screen with the RRI length while the strip is in the middle of the screen.

E. Label the "true" shortest RRI (N-N beats, not S-N beat) by pressing the "F" FILE KEY and the "F" again to allow you to write in "Shortest RRI". This will be recorded on diary and on cover page of report. Press RETURN KEY to get back to histogram

F. Once a "true" shortest RRI is found, you will move to the Longest RRI by pressing > KEY while viewing the histogram. Press "S" KEY to view strip with this RRI.

G. Continue as you did for the shortest RRI, making sure to relabel all beats.

H. Once a "true" longest RRI if found, press " $F$ " to file it, and "F" again to write in "Longest RRI."

I. Press " $L$ " to have longest RRI placed on cover sheet

J. Press "M" to eliminate RRI filled with artifact from histogram. Press RETURN KEY to view histogram

K. While viewing histogram, press "F" File, to file histogram.

L. Press RETURN KEY to return to HISTOGRAM MENU SELECTION,

M. Press ESC KEY to return to MAIN MENU

VII. Report Review

A. Select \#9-Report Review from MAIN MENU

B. Verify patient record you want to review is in slot 0 .

C. Press H (HRV) to print one copy of the Heart Rate Variability. Screen will go blank, Numbers 1 to 3 will light up in sequence. Once 3 lights up, a HRV frequency graph will be depicted. Press ESC KEY and you will return to MAIN MENU.

D. Select \#9-Report Review from MAIN MENU

E. Select R (Review) to review a report.

F. Select report number of patient (should be 0 if current report) and press RETURN KEY

G. Enter "1" when prompted to select FORMAT 1. Will automatically show report without return key being used.

H. You will see diary first. Skim to see that entries are correct and that shortest and longest RRI are noted on diary.

I. Press PF4 to move to next page.

J. Cover page will include sampling length (should be $24 \mathrm{hr}$ unless holter removed early) and low/high/average HR. Press PF4 to move to next page.

K. Scroll down next page to see how the duration of each hour (DCN reduces duration in each hour). Program automatically substitutes the next normal RRI into the DCN area) Note hours in which duration is less than 30 minutes and include under analyst comments on last page of report. Press PF4 to move to next page.

L. Continue reviewing report until reaching last page. Document quality of tape (excellent, very good, good, fair, poor) and analysis time in minutes. Enter your initials and monitor number if not already there. Under analyst comments include DCN, hours of duration less than 30 minutes, total duration $<24$ hours should be indicated if occurs. Press PF4 to move to next page.

M. Prompt will appear asking if you want to save report--Press $Y$

N. Prompt will appear asking if you want to print report-Press $Y$

O. Prompt will appear asking how many copies of report to print-Press 1

P. Prompt will appear asking how many strip copies-Press 0

Q. Press RETURN KEY. Report will print. Press RETURN KEY TO get back to MAIN MENU.

R. Select \#1 NEW TAPE from MAIN MENU and press RETURN KEY

S. Select "D" for delete from NEW TAPE MENU and enter number of tape to be deleted

T. You will be asked if you are sure $(\mathrm{Y} / \mathrm{N})$ select appropriate letter. 
U. Strips will be saved to hard drive (report was previously saved) and slot is now available for new tape to be entered into that space for analysis

VIII. Files

A. Calculate low/high ratios for HRV

B. Make one copy of entire report for lab

C. Place original in principle investigator's folder

D. Place copy for lab in outer office of AFT lab

IX. Troubleshooting

A. Playback error: Tape will not load

1. remove tape from cassette reader in hard drive, reinsert, and press return

2. select playback error from MAIN MENU

B. Diary times incorrect

1. verify start time and date are correct in \#2-Patient information

2. check trends or full disclosure (enter time on full disclosure menu that you want to look at and it will show that screen). If there is no data on the tape for that time, a forward time cannot be entered in the diary section. 


\title{
Appendix B
}

\section{Informed Consents for Patients with Renal}

\author{
Coneen to Partidpate In the Study \\ "Antanomle Function and Poittracipont Quilto of Lite"
}

\section{A. PURPOSE FOR THE RESEAPCH AND PROCEDURES}

The "Autonomic Function and Postransplant Quality of Liffe" study is a rerearch project being dose to help understand bow changes in the body's atuonomic system affect quifity of life, The autonoxnic systexn is responatible for many body functions that go on antomatically. Some of the things the autonomic system does inchyles regulaling our heart rate, cansing our blood vessobs to constrict and relax at the right limes, mintaining orr blood pressure regandless of our body position, and cointrolling same of our glomash ectivity. When a person has ridney dinease er diaberes, the autonomic system often is not . able to carry out its job as well us before. When this happens the persorn may have to change some of theil daily activities and adjust his or her lifestyle.

The study being done is looking at bow changes in the gulonornic system affect quality of life hefore and after kidney and panceras-lidney transplantation. By underatanding bow the artonomic syatem affects qualizy of life we will be able to develop now ways of caring for putients thet can improve their life following transplentation.

If you agree to help with our surdy you will undergo some special wests before your transplant and at six, twelve, ond twenty-torr months after your transplant. These tests are listed below.

1. Quality of Hfe questionnaire-You will be given sewarl questionngires that ask queations about your lifeatyle, any adjuimente you've had to make in your life, and how satisfied you are with your life.

2. Blood vesed tests-A small fint matal probe will be toped to your middle finger and outuched to a barge machine. This probe measimer the blood flow in your finger by uging infraned light waves. Thea are no deedles ured with this text $Y_{\text {ou }}$ will be sitting in 1 comfartable cheir while the probe is on your finger and we will be ast you to do geverol things. First, we will just recond the blood flow in your fingor while you are sitting quisaly. Second, we will ask you to raise your arm while we recond the blood flow. Thind. we will atk youi to placo your hand in a basin of loe water for one minute and we. will tate recridings before and after this.

3. Blood pressure testr-For these ratg you will have a blood pressure coff pat on your arm and fitached to a machime that wotoroulically reconds your blood pressure. Yon will also heve condiogram palchss typed to your chest to recond your heirt beats. We will also ask you to do several things for these bate. First, we will just record your heart rate and blood prosore whlle you are sinting quietly. Second, we will at you to blow into a small machine is hard as you cen for cos minn'e whlle we talte recordinge. Thind, we will talke recurdings beform and fifter you rtand up.

4. Heart tests-For this test you will be wed to wagr a "Holter" monitor for 24 hours. This monitoc constantly neconds your hedrt beat on a tape reconding. For this best you will have the cardiogran patches taped to your chest and will cany a irecrating machine the size of a small purse for 24 boors.

Pitiont Inftials 
Antonowic Fusction and Quality of Life Stucty

5. Stomach tests-Two tests will be used to mensure your stomach function. First, we will ast you to anower a gaestiomnine that asks ebout the find of problems you may have had with your atomach. Second, we will tape a miall probe just jiko the cardiogram patches over your stomach. This will recosd the activity of youl gtornach just like a cardiogram necords the actluity of your heart

6. If you have diabetes we will teat your blood augar before the test. If you hove low or high blood sugar the ustal steps you talos to conroct this problem will be thisen. If your blood sugar is aver $200 \mathrm{mg} / \mathrm{d}$ we will also bake your blood gugar at the ead of the test and if newessary try to schedule another west fox you when your blood sugar is lower.

\section{B. Rusks}

The most uncomfortable part of this pert of this study is the ice water test whene you are asted to keep your hand in a besin of ke water for one mimule.

\section{WHO TOCONTACT WITH QUESTXONS}

Any time during this study, if you have a question about the study or your dights as a subject contact D. Donna Hathaway it (901) 4.48-5924.

\section{BENEFIS OP THIS RESEARGH}

Knowing how changes in the atunnomic system brought about by transplantation aticc quality of life will belp us find oew whys to improve the lives of palients with bidnoy dience and dishetex Patients helping with the stndy will also benefit from the thorough examination of their anionomic finction. Mnny patients with kidmey disease and/or dabetes suffer from poor anturnenic function. In ane cases abnomal autoonic function can lead to severe blood vesell, beart, or strmosh problems. By helping with this study and having these tests we may be able to identify some patients who have early problems with their watonomic function.

\section{B. AltERnATYE ProchiYURES}

There ane wo ofher besta avallable that provide as thorough an examination with as liute discomfort for the patient.

\section{F. CONFDARTINIIT}

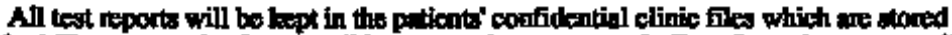
In a locked file noom and only accessible to traneplant personnel. Data from the reports will be copiled onto compuler wortsheets which identify the pattent by a number. No patient helping with this ofudy will be identified by nam:

Pationt Initials 
Ausiomic Function and Quality of Life Sndy

\section{G. LEOAL RWGTS}

I understand that I am not waiving any legal rights or releasing the boopilol or its afenes from liability for degligence. I undersund that in the event of physical injury resulting from the research procedures, the Univensity of Tentreseed does not have funds burlyeted for compensetion either for lost wages or for medical treatment. Therefore, the University does not provide for treatment or meimbursempents for such injuries.

\section{H. VOUNTARY CONBENT}

I have read the description of the "Autnnomic Function and Quality of Life Strdy" and I have freely volunteered to partichipele in it. I have hed possible side effects, adverse resctions, and alternatives explained. I have bad an opportuatity to ask geestions and have mocived aceeptahle answers, I understand that I may withdraw from this study at any time and I will still recelve standard treatruedt for my condition. 
Consent to Particlpats in the Study

"Antonomile Function ani Posturanoplant Quality of Lthe"

\section{Banlfly Stody Partictpantr}

\section{A. FURPOSE FOR THE RESEARCF AND PMOCEDURES}

The "Autonomic Function and Portramsplant Quality of Life" study is a reseanch project being done to help understand how changes in the body's antonomic system affect quality of hife. The antrnoxnic system is responsible for many body functions that go on automatically. Some of the things the autronomic system dow inchides retgulating pur heart Itte catnsing our blood vessels to condrict and relar at the right times, maintaining our blood pressure regandless of our body position, and controlling some of our stomach ectivity. When a person has kidnsy tisease or diaheres, the amtnomic system oftern is not ahls to carry out its job as well as before. When this happens the persen may have to change some of their daily activities mol adjust his or her lifestyle.

The study being dome is looking at how changes in the autonomic system affect quality of life before and after bifney and pancesas-kidney trankpiantation By understandiag how the anmomic system affects gality of life we will be able to develop new ways of caring for pationts that can improve their lifo following transplantation. In onder to detasmine how transplantation alhes peoples' lives, we must also collect information from people who are not laving a transpinm.

If you agree to balp with our stedy you will undergo soune special bests. These vests will be performed only once unless you arts ahle and agree have them reperied one week later. Theose tests ane listed below.

1. Blood worts-A smail flat metal probe will be baped to your middle finger and atrached to a larpe machine. This probe mensented the blood flow in your finger by using : infrited light waves. There are no nowdles need with this tost You will be silting in a comfortahile chair while the ponles is on your finger and we will be agk you to do several things. First, we will inst record the blood flow in yoar fingor while you are sitting quietly. Second, we will akt you to raise your mon while we record the bloot flow. Third, we will esit you to place your hand in a basin of ics water for one minuter and we will gitse recordings befure and aftrir this.

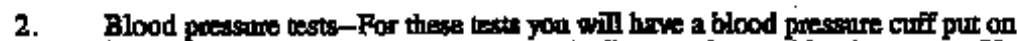

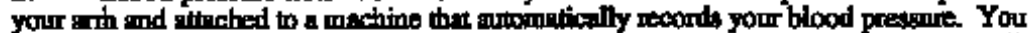
will abo have cariioeram pathes toped to your chest we recod your beart beals. We will aiso ask you in do several things for these teas Fist, we will just record your heart rate and blond prestrme while you are sitring quictly. Second, we will ast you to blow into a small machine as hard as you can for ons minme while we toles recordingh. Third, we will vike recordings befoes and after you stind op.

3. Heart tests-For this test you will be athed to wear a "Hilpes" monitor for 24 hours This monitor constimtly nexords your heart beat on a tape recording. For this tesx you whil

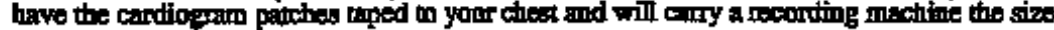
of a small purse for 24 bours.

Participent Initivis 
4. Scomach wests-We will tape a sonall probe just fike the cendiogram patches over your stomach. This will recoct the activity of your stomach jagt like a cardiogram neconds the activity of your heart

B. RUSxs

The most uncomfortable part of this part of this stmdy is the ice water test where you are agked to keep your band in a basin of ice waler for one mimube.

\section{c. WHO TO CONTACT WTH QUESTIOSS}

Any time furing this stady, if you have a question about the study ar your tights as a salbject contact De. Donina Hathawisy at (901) 448-5924.

\section{BENEFIS OF THIS RESEARCH}

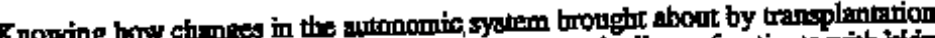
affect quality of life will betp as find new ways to improve the lives of patients with itsiney

- Feos and diabetes. Persons helping with the sindy will also benefit by having a ares abrormal ansonomic funcion can lead to severe blood vessel, heart, or stomiach poblems.

\section{E. ALTERNATIVE PEOCEITURBS}

There are no other terts aviilabie that provide as thorough an examination with as bitte jibeconfort for the patient

\section{F. CONFIDENTIALITY}

All weports will be kept in confidential files which are stored in a locired file mom ondy accessible on research persolmel Dam from the reports will be copiad onto computer wodkshests which identify the participants by a momber. No one belping with this stody will be identified by name.

\section{G. LEGAL RFCFIS}

I umierstond that I am not wriving any leqgl rights or releasing the hospital $x$ iss a

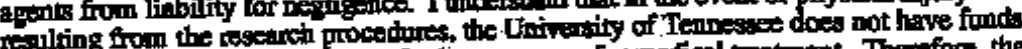

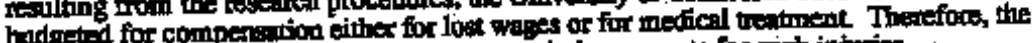
University does not provide for treatment or teimborsements for scich infurivs.

\section{H. Vounstary Cossent}

I have read the decicription of the "Antuninmic Function and Qpatily of Liffe Stody" and I have frecly volunterred to participate in it. I have had posihte side effects, anverse

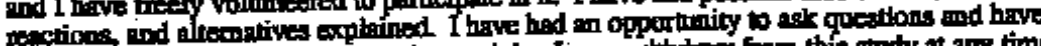

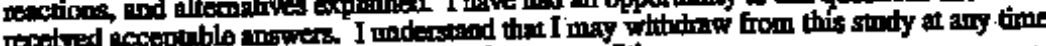
gnd I will still receive standend to:

Investigetor

Wituss

\section{Fateon}

Dits 


\section{Appendix C}

\section{Autonomic Symptom Checklist}

Please tell us how much of a problem the following symptoms have been to you during the last month. Circle one number between 0 and 6 . The number 0 means the symptom is never a problem to you and 6 means the symptom is always a problem to you.

1. Symptoms when you stand:

\begin{tabular}{ccccccc} 
Never & & \multicolumn{4}{c}{ Always } \\
0 & 1 & 2 & 3 & 4 & 5 & 6 \\
0 & 1 & 2 & 3 & 4 & 5 & 6 \\
0 & 1 & 2 & 3 & 4 & 5 & 6 \\
0 & 1 & 2 & 3 & 4 & 5 & 6 \\
0 & 1 & 2 & 3 & 4 & 5 & 6 \\
0 & 1 & 2 & 3 & 4 & 5 & 6
\end{tabular}

Comments:

2. Symptoms when you sweat:

Never

Always
a. Do you have an increase in sweating in any part of your body?
b. Do you have a decrease in sweating in any part of your body?
c. Do you sweat during or after eating?
d. How much of a problem are these symptoms for you?

$\begin{array}{lllllll}0 & 1 & 2 & 3 & 4 & 5 & 6\end{array}$

$\begin{array}{lllllll}0 & 1 & 2 & 3 & 4 & 5 & 6\end{array}$

$\begin{array}{lllllll}0 & 1 & 2 & 3 & 4 & 5 & 6\end{array}$

$\begin{array}{lllllll}0 & 1 & 2 & 3 & 4 & 5 & 6\end{array}$

Comments:

3. Symptoms of bowel and stomach problems:

\begin{tabular}{lllllll} 
Never & \multicolumn{4}{c}{ Always } \\
0 & 1 & 2 & 3 & 4 & 5 & 6 \\
0 & 1 & 2 & 3 & 4 & 5 & 6 \\
0 & 1 & 2 & 3 & 4 & 5 & 6 \\
0 & 1 & 2 & 3 & 4 & 5 & 6 \\
0 & 1 & 2 & 3 & 4 & 5 & 6 \\
0 & 1 & 2 & 3 & 4 & 5 & 6
\end{tabular}

Comments:
a. Do you have diarrhea?
b. Do you have diarrhea at night?
c. Do you lose control of your bowel movements?
d. Do you have trouble moving your bowels?
e. Do you have stomach fullness before you are finished eating?
f. How much of a problem are these symptoms for you?

$\begin{array}{lllllll}0 & 1 & 2 & 3 & 4 & 5 & 6\end{array}$ 
4. If you are a man, please answer the following:

a. Are you able to get an erect penis?

b. Are you able to have an erect penis when you want one?

Comments:

c. Are you able to maintain the erection as long as you want?

d. Over time, have you noticed any changes in the firmness?

e. How much of a problem are these symptoms for you?

\begin{tabular}{cccccccc}
\multicolumn{1}{c}{ Never } & \multicolumn{4}{c}{ Always } \\
0 & 1 & 2 & 3 & 4 & 5 & 6 \\
0 & 1 & 2 & 3 & 4 & 5 & 6 \\
0 & 1 & 2 & 3 & 4 & 5 & 6 \\
0 & 1 & 2 & 3 & 4 & 5 & 6 \\
0 & 1 & 2 & 3 & 4 & 5 & 6
\end{tabular}
$\begin{array}{lllllll}0 & 1 & 2 & 3 & 4 & 5 & 6\end{array}$
Never Always
$\begin{array}{lllllll}0 & 1 & 2 & 3 & 4 & 5 & 6\end{array}$
$\begin{array}{lllllll}0 & 1 & 2 & 3 & 4 & 5 & 6\end{array}$
$\begin{array}{lllllll}0 & 1 & 2 & 3 & 4 & 5 & 6\end{array}$

Never Always

5. If you have diabetes, please answer the following:
a. Do you have symptoms when your blood sugar is low?
$\begin{array}{lllllll}0 & 1 & 2 & 3 & 4 & 5 & 6\end{array}$
b. Have you had an increase in symptoms
$\begin{array}{lllllll}0 & 1 & 2 & 3 & 4 & 5 & 6\end{array}$
c. Have you had a decrease in symptoms
$\begin{array}{lllllll}0 & 1 & 2 & 3 & 4 & 5 & 6\end{array}$
d. Does it take a lower blood sugar for you to feel symptoms
$\begin{array}{lllllll}0 & 1 & 2 & 3 & 4 & 5 & 6\end{array}$
e. How much of a problem is this for you?
$\begin{array}{lllllll}0 & 1 & 2 & 3 & 4 & 5 & 6\end{array}$

Comments:

6. How many drinks of beer, wine, or liquor do you have most days?

7. How many servings of caffeine (soft drinks, coffee, tea, chocolate) do you have most days?

8. Do you use tobacco products? Mark with an X all the ones you use.
(a) Yes No
_ (b) cigarettes
_ (c) cigars
- (d) chewing tobacco
- (e) snuff
_ (f) pipes

9. Please list all the medications you are taking.

Akc
$2^{\text {nd }}$ revision
Summer 1996 


\section{Appendix D}

\section{Calculation Procedure for the Autonomic Symptom Checklist}

There are 3 tabulations for each individual for each category. They are raw symptom score/category, symptom score/category, and impact score/category.

1. Raw Symptom Score-sum of all points for that category excluding the last question, which is the impact, question. The range of scores differs per category: hypotension=0-30, sudomotor=0-18, GI=0-30, impotence=0-24, hypogycemia=0-24. In all categories a higher scores indicate increased symptomatology.

2. Symptom Score-the Raw Symptom Score divided by the number of questions possible in the category. Range is $0-6$. In all categories a higher scores indicate increased symptomatology.

3. Symptom Impact Score-points indicated by subject on the impact question. Range is $0-6$. The higher the score the more that category of symptoms is a problem to the subject.

4. Other scores

- Raw Total Symptom Score-Sum of all Raw Symptom Scores for a subject. The range of the Raw Total Symptom Score differs by group, thus they can not be compared. Diabetic women=102, diabetic men=126, nondiabetic women=78, and nondiabetic men $=102$.

- Total Symptom Score-Sum of category symptom scores divided by the number of categories available for that group to answer (eg., DM women=4, DM men=5, NonDM women=3, NonDM men=4). The possible range for each group is $0-6$, thus the scores can be compared across groups.

- Raw Total Impact Score-Sum of all points scored on impact questions. The range varies per group, thus it can not be analyzed across groups.

- Total Impact Score-Sum of the category impact scores divided by the number of impact questions possible for that group. The possible range for each group is $0-6$, thus the scores can be compared across groups.

- Raw Index Score-Raw Total Symptom Score multiplied by the Raw Total Impact Score. The range of the Raw Index Score differs by group, thus, they can not be compared across groups.

- Index Score-Total Symptom Score multiplied by the Total Impact Score. The possible range for each group is $0-36$, thus, the scores can be compared across groups.

akc

Revision update 4/16/98 


\section{VITA}

Ann King Cashion was raised in Helena, Arkansas where she graduated from

Central High School in 1974. The following September she entered the University of North Carolina at Chapel Hill and in May 1978 received the degree of Bachelor of Science in Nursing. While working as a critical care nurse in a private hospital she completed her Master of Nursing Science degree at the University of Arkansas Medical Sciences in Little Rock, Arkansas in 1982. Ann worked as a Critical Care Clinical Nurse Specialist for 10 years before entering the Doctor of Philosophy Program at the University of Tennessee, Memphis, where she received her PhD in Nursing in 1998. 